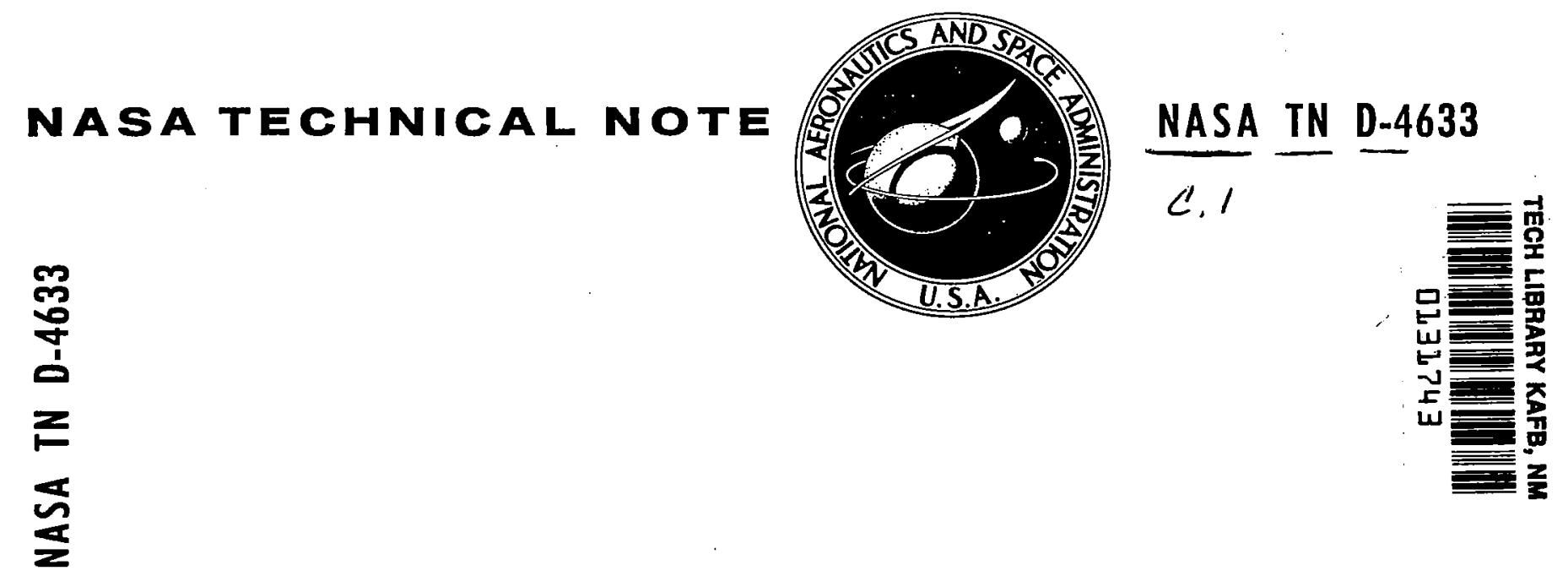

LOAN COPY: RETURN TO AFWL (WLL-2)

KIRTLAND AFB, $N$ MEX

\title{
COUPLED-CHANNELS METHOD FOR REARRANGEMENT COLLISIONS
}

by Howard C. Volkin

Lewis Research Center

Cleveland, Obio

National aERONaUtics AND SPACE AdMINISTRation • WASHINGTON, D. C. • JULY 1968 


\section{COUPLED-CHANNELS METHOD FOR}

\section{REARRANGEMENT COLLISIONS}

By Howard C. Volkin

Lewis Research Center

Cleveland, Ohio

NATIONAL AERONAUTICS AND SPACE ADMINISTRATION 


\begin{abstract}
The classification and construction of open-channel projection operators for a given rearrangement collision are developed from the unifying viewpoint of their projective spaces in the total Hilbert space of the system. The representative case of the pickup or stripping process is treated. The open-channel projection operators are constructed from the channel-subspace projectors with the help of generalized channel transformation functions. Various projection operators that can be obtained in closed form are identified. The use of the projection operators to obtain coupled equations describing the reaction is discussed, including their application to generalized potential models.
\end{abstract}




\title{
COUPLED-CHANNELS METHOD FOR \\ REARRANGEMENT COLLISIONS
}

\author{
by Howard C. Volkin \\ Lewis Research Center
}

\begin{abstract}
SUMMARY
As shown by Feshbach, coupled-channel equations that describe a reaction can be obtained by means of an open-channel projection operator. For a given rearrangement collision, the classification and construction of such operators are developed herein from the unifying viewpoint of their projective spaces, which are subspaces of the total Hilbert space of the system. The open-channel projection operators are not unique, but the practical limitations on their choice are made clear when their construction is viewed in terms of the fundamental channel subspaces that arise naturally in the problem. The projectors for the channel subspaces are the basic ones from which the open-channel projectors are constructed. Generalized channel transformation functions supply all the information needed for this purpose. The representative case of the pickup or stripping process is treated. The various possible projection operators that can be obtained in closed form are identified for the two-channel case; these include those given by Feshbach and by Chen and Mittleman, as well as new ones suggested by the methods presented herein. In all cases, the extension to the multichannel case is shown to follow easily. The use of projection operators to obtain the coupled equations is discussed, including their application to generalized potential models. The appropriate boundary conditions are also given in each case.
\end{abstract}

\section{INTRODUCTION}

Rearrangement collisions have received considerable study in the last few years. Within the framework of his general reaction theory, Feshbach (ref. 1) has shown how any reaction can be described exactly by a system of Schrödinger-type equations. The equations couple together various functions, each of which yields the asymptotic behavior of the wave function in its associated channel (or group of channels). The set of coupled 
equations is obtained from an open-channel projection operator $P$, in terms of which Feshbach's unified reaction theory is formulated. The projection operator $\mathbf{P}$ that selects the open channels is not unique. When this method is applied to reactions involving simple incident and target particles (e.g., electrons (positrons) on light atoms) or to more complicated target systems described by generalized potential models, it leads to a system of integrodifferential equations whose accurate numerical solution is within the capacity of a large, high-speed computer.

For inelastic scattering, the coupled-channels method has proved to be useful, and is developed naturally from the unique expansion of the scattering state in the set of orthogonal states of the target system. By the simple projecting out of the open-channel components of the expansion, a system of coupled equations for these components is obtained. In rearrangement collisions, however, the final noninteracting states in the rearrangement channels are not orthogonal to those in the direct channels, and there is more arbitrariness in the choice of a projection operator. In a given problem, any suitable projection operator will yield a set of coupled equations that is formally exact. A projector whose coupled equations have a form convenient for applied computations is desired. Such projectors exist and can be expressed in a closed form. They offer a practical way of treating rearrangement collisions that is free of the approximations commonly used heretofore in such problems.

Among the explicit projectors given by Feshbach (ref. 1) to illustrate various facets of his theory is one applicable to the case of pickup or stripping reactions. The associated coupled equations are also discussed. Mittleman (ref. 2) has given another projection operator for this reaction, one designed to yield coupled equations that are easier to apply than those of Feshbach. Discussions relevent to Mittleman's projector are presented in papers by $\mathrm{Coz}$ (ref. 3) and Hahn (ref. 4). Mittleman's first result was somewhat disappointing, however, inasmuch as an auxiliary integral equation problem must be solved to obtain the explicit expression for his projector. Recently, Chen and Mittleman (refs. 5 and 6 ) have shown how to circumvent the difficulties in constructing projectors of this type.

Herein, the treatment of projection operators for rearrangement collisions is formulated rather differently from any given previously. Basically, the problem is viewed in terms of the subspaces (in the total Hilbert space of the system) that arise naturally from the various sets of internal states occupied by the channel fragments. These channel subspaces provide the fundamental projectors. For every pair of open channels, where the fragments of one channel involve a rearrangement of the others fragments, a general transformation function is defined. This transformation function is the scalar product between two state vectors of the total system. The function contains not only the overlap integral of the fragments' internal states, but also the required kinematic connection between the coordinates describing the relative motion of the fragments in the different 
types of channels. The transformation functions contain all the information needed to construct the open-channel projection operators.

The description in terms of the channel subspaces, besides furnishing a basis for the construction of projection operators, reveals the relations between the various projectors that have already been proposed, suggests new ones, and shows the limitations on their possible types. In a previous work (ref. 7), a general class of open-channel projection operators for any given reaction was described. This class, which may well include most of the useful ones for computational applications, consists of every projector whose projective space contains, in its entirety, the channel subspace of every open channel. Projection operators of the type given by Mittleman and by Feshbach can be characterized as having certain minimal and maximal projective spaces, respectively, in the class.

In the succeeding sections of this report, the general formalism is explained, and certain transformation functions are discussed. The methods are illustrated by a simple example (the two-channel projector first given by Mittleman), various two-channel projectors that can be obtained in closed form are constructed, and the generalizations to the multichannel case are shown to follow easily. Finally, the asymptotic properties that provide the boundary conditions for the coupled equations are given, followed by brief discussions of the equations themselves.

\section{BASIC THEORY AND FORMALISM}

\section{Channel Subspaces}

The various channel projection operators that occur in a given rearrangement collision are developed from the unifying viewpoint of their projective subspaces in the full Hilbert space of the problem. The pickup (or stripping) reaction serves as the typical rearrangement process in the considerations. For simplicity, it is assumed that only two types of channels are open. In addition, recoil effects and the effects of identity are neglected. Thus, the target is taken to be infinitely massive and all particles are treated as distinguishable. The notation used herein (see the appendix) is based on that of reference 7, which may be consulted for further details. Although the notation appears somewhat cumbersome, it has the advantage of exhibiting explicitly the variables that are employed at any given point and generalizes directly to more types of open channels or to other kinds of reactions.

The symbols ( $\mathscr{A}-1), \mathscr{A}$, and $\mathscr{D}$, respectively, are for the heavy core nucleus, the nucleus consisting of particle $p$ bound to the core, and the bound system of particles $\mathrm{n}$ and $\mathrm{p}$. With position vectors measured relative to the fixed center of $(d-1)$, let $\vec{r}_{0}$ and $\vec{r}_{1}$ specify the positions of $n$ and $p$, respectively. The set of variables for the particles that constitute the core nucleus are represented simply as $\vec{\xi}=\left(\overrightarrow{\mathbf{r}}_{2}, . ., \overrightarrow{\mathbf{r}}_{\mathbf{A}}\right)$. 
The center of mass of $\mathscr{D}$ is at $\vec{R}=(1 / 2)\left(\vec{r}_{0}+\vec{r}_{1}\right)$ when the masses of $n$ and $p$ are equal. The internal motion is a function of $\vec{r}=\vec{r}_{0}-\vec{r}_{1}$. Channel $\alpha \mathrm{m}$ consists of particle $\mathrm{n}$ and nucleus $\mathscr{A}$, where $\mathscr{A}$ is in the $\mathrm{m}^{\text {th }}$ excited state with wave function $\phi_{\mathrm{m}}\left(\overrightarrow{\mathrm{r}}_{1}, \vec{\xi}\right)$. Channel $\beta \mathrm{n}$ consists of the fragments $\mathscr{D}$ and $(\mathscr{A}-1)$ in the states of internal motion given by the wave functions $\chi_{i}(\vec{r})$ and $\psi_{j}(\vec{\xi})$, respectively. The index $n$ specifies the pair $(i, j)$ and is ordered toward increasing values of the total internal energy of the fragments. The notation $(\chi \psi)_{n}$ is usually written for $\chi_{i} \psi_{j}$.

Consider now the scattering state $\Psi$ for the pickup reaction. Large separations of the particles in the $\alpha$ channels correspond to large values of $r_{0}$, while $r_{i} \lesssim R_{A}, i=1$, $2, . . . \mathscr{A}$; correspondingly, in the $\beta$ channels, $\mathrm{R}$ becomes large, while $\mathrm{r} \lesssim \mathrm{R}_{\mathrm{D}}$, $\mathrm{r}_{\mathrm{i}} \lesssim \mathrm{R}_{\mathrm{A}-1}, \mathrm{i}=2, \ldots, \mathscr{A}$. Here, $\mathrm{R}_{\mathrm{A}}$ is some effective radius of the nucleus $\mathscr{A}$, and so on. If the energy is such that there are $\mathrm{M}^{\mathrm{O}}+1$ open $\alpha$ channels and $\mathrm{N}^{\mathrm{O}}+1$ open $\beta$ channels, then the asymptotic behavior of the wave function is shown as follows:

$$
\begin{aligned}
& \left\langle\overrightarrow{\mathrm{r}}_{0}, \overrightarrow{\mathrm{r}}_{1}, \vec{\xi} \mid \Psi\right\rangle \vec{\alpha}^{(2 \pi)^{-3 / 2}} \sum_{\mathrm{m}=0}^{\mathrm{M}^{\mathrm{o}}} \phi_{\mathrm{m}}\left(\overrightarrow{\mathrm{r}}_{1}, \vec{\xi}\right)\left[\delta_{\mathrm{mo}} \mathrm{e}^{\mathrm{i \vec {k } _ { 0 }} \cdot \overrightarrow{\mathrm{r}}_{0}}+\mathrm{A}_{\alpha \mathrm{m}}\left(\hat{\mathrm{k}}_{0}, \hat{\mathrm{r}}_{0}\right) \frac{1}{\mathrm{r}_{0}} \mathrm{e}^{\mathrm{i \textrm {k } _ { \mathrm { m } }} \mathrm{r}_{0}}\right] \\
& \langle\overrightarrow{\mathrm{R}}, \overrightarrow{\mathrm{r}}, \vec{\xi} \mid \Psi\rangle \vec{\beta}^{(2 \pi)^{-3 / 2}} \sum_{\mathrm{n}=0}^{\mathrm{N}^{\mathrm{o}}}[\chi(\overrightarrow{\mathrm{r}}) \psi(\vec{\xi})]_{\mathrm{n}} \mathrm{A}_{\beta \mathrm{n}}\left(\hat{\mathrm{k}}_{\mathrm{o}}, \hat{\mathrm{R}}\right) \frac{1}{\mathrm{R}} \mathrm{e}^{\mathrm{i} \kappa_{\mathrm{n}} \mathrm{R}}
\end{aligned}
$$

The wave vector $\overrightarrow{\mathrm{k}}_{0}$ specifies the incident plane wave in channel $\alpha 0$, and $\mathrm{k}_{\mathrm{m}}$ and $\kappa_{n}$ are the wave numbers for the relative motion of the free fragments in the channels $\alpha \mathrm{m}$ and $\beta \mathrm{n}$, respectively. The transition amplitude for channel $\beta \mathrm{n}$ is $\mathrm{A}_{\beta \mathbf{n}}\left(\hat{\mathrm{k}}_{0}, \hat{\mathrm{R}}\right)$, and so forth.

The full scalar product of the Hilbert space is formed by integration over a complete set of particle coordinates. Any two of the coordinates $\vec{r}_{0}, \vec{r}_{1}, \vec{r}$, and $\vec{R}$ along with the coordinates $\vec{\xi}$ for the core particles can be members of a complete set. Coordinates other than position vectors will not be indicated explicitly. If the pair $\left(\vec{r}_{0}, \vec{r}_{1}\right)$ is used, the volume element for the scalar product integral is $d \tau=d \vec{r}_{0} d \vec{r}_{1} d \vec{\xi}$. The Jacobian of the transformation from the pair $\left(\vec{r}_{0}, \vec{r}_{1}\right)$ to the pair $(\vec{R}, \vec{r})$ has the value (-1). Accordingly, the volume element can also be written as $d \tau=d \vec{R} d \vec{r} d \vec{\xi}$, and the coordinate basis vectors can be taken to satisfy the relation $\left|\vec{r}_{0}, \vec{r}_{1}, \vec{\xi}\right\rangle=|\vec{R}, \vec{r}, \vec{\xi}\rangle$. The pair of coordinates $\left(\vec{r}_{0}, \vec{r}_{1}\right)$ may be called the "natural pair" for the $\alpha$ channels. The variable $\vec{r}_{1}$ is the convenient internal coordinate for the nucleus . The uniform motion of the fragments when their separation becomes sufficiently large that they no longer interact is 
expressed most simply in terms of $\vec{r}_{0}$, the relative separation between their mass centers. Consequently, the wave function representative (eq. (1)), based on the choice $\left(\overrightarrow{\mathrm{r}}_{0}, \overrightarrow{\mathrm{r}}_{1}\right)$, attains asymptotically in the $\alpha$ channels the form of an eigenstate of the noninteracting Hamiltonian for the $\alpha$ channels. For the $\beta$ channels, the asymptotic form (eq. (2)) shows that the pair $(\vec{R}, \vec{r})$ is the natural one. The formalism is first developed in terms of the natural pairs of channel coordinates. When it becomes necessary to use other coordinate pairs, only slight extensions of the formalism are required. the form

Let $5_{\alpha \mathrm{m}}$ be the subspace of the total Hilbert space that is spanned by all states of

$$
\left|\rho \phi_{\mathrm{m}}\right\rangle=\int \mathrm{d} \overrightarrow{\mathrm{r}}_{0}^{\prime} \rho\left(\overrightarrow{\mathrm{r}}_{0}^{\prime}\right)\left|\overrightarrow{\mathrm{r}}_{0}^{\prime}, \phi_{\mathrm{m}}\right\rangle
$$

where $\rho\left(\overrightarrow{\mathrm{r}}_{0}\right)$ represents any state of motion for the mass centers of the $\alpha$ fragments, that is, for particle $\mathrm{n}$ relative to nucleus $\mathscr{A}$. Parentheses are used to indicate a scalar product taken over a set of variables that is not a complete set for the total system; thus, $\rho\left(\overrightarrow{\mathrm{r}}_{0}\right) \phi_{\mathrm{m}}\left(\overrightarrow{\mathrm{r}}_{1}, \vec{\xi}\right)=\left\langle\overrightarrow{\mathrm{r}}_{0}, \overrightarrow{\mathrm{r}}_{1}, \vec{\xi} \mid \rho \phi_{\mathrm{m}}\right\rangle=\left(\overrightarrow{\mathrm{r}}_{0} \mid \rho\right)\left(\overrightarrow{\mathrm{r}}_{1}, \vec{\xi} \mid \phi_{\mathrm{m}}\right)$. The projection on the subspace $5_{\alpha \mathrm{m}}$ is given by the operator

$$
\begin{gathered}
\left.\Pi_{\alpha \mathrm{m}}=1_{\mathrm{r}_{0}} \mid \phi_{\mathrm{m}}\right)\left(\phi_{\mathrm{m}} \mid\right. \\
\Pi_{\alpha \mathrm{m}}=\int\left|\overrightarrow{\mathrm{r}}_{0}^{\prime}, \phi_{\mathrm{m}}\right\rangle \mathrm{d}{\overrightarrow{\mathrm{r}_{0}^{\prime}}}_{0}\left\langle\overrightarrow{\mathrm{r}}_{0}^{\prime}, \phi_{\mathrm{m}}\right|
\end{gathered}
$$

The closure integral over the coordinate $\vec{r}_{0}$ in equation (4) represents the unit operator $\mathbf{1}_{\mathbf{r}_{0}}$ in the space of all states $\rho$ of relative motion, which is called the $\overrightarrow{\mathbf{r}}_{0}$ space. Similarly, let $\mathfrak{S}_{\beta \mathrm{n}}$ be the subspace spanned by states of the form $\sigma(\chi \psi)_{n}$, where $\sigma(\overrightarrow{\mathrm{R}})$ represents any state of relative motion for the particles $\mathscr{D}$ and $(\mathscr{A}-1)$ of the $\beta$ channels. The projector for $\mathfrak{s}_{\beta \mathrm{n}}$ is

$$
\begin{aligned}
\Pi_{\beta \mathrm{n}} & \left.=1_{\mathrm{R}} \mid[\chi \psi]_{\mathrm{n}}\right)\left([\chi \psi]_{\mathrm{n}} \mid\right. \\
& =\int\left|\mathrm{R}^{\prime},[\chi \psi]_{\mathrm{n}}\right\rangle \mathrm{dR}^{\prime}\left\langle\mathrm{R}^{\prime},[\chi \psi]_{\mathrm{n}}\right|
\end{aligned}
$$

The scattering state $\Psi$ can be expanded in terms of the complete set $\phi_{\mathrm{m}}$ (which includes states belonging to the continuum spectra of $\mathscr{A}$ ). The $\mathrm{m}^{\text {th }}$ term in the expansion, namely $\Pi_{\alpha \mathrm{m}} \Psi=\mathrm{U}_{\mathrm{m}} \phi_{\mathrm{m}}$, where $\mathrm{U}_{\mathrm{m}}$ is represented by 


$$
\mathrm{U}_{\mathrm{m}}\left(\overrightarrow{\mathrm{r}}_{0}\right)=\left\langle\overrightarrow{\mathrm{r}}_{0}, \phi_{\mathrm{m}} \mid \Psi\right\rangle=\int \phi_{\mathrm{m}}{ }^{*}\left(\overrightarrow{\mathrm{r}}_{1}^{\prime}, \vec{\xi}^{\prime}\right) \Psi\left(\overrightarrow{\mathrm{r}}_{0}, \overrightarrow{\mathrm{r}}_{1}^{\prime}, \vec{\xi}^{\prime}\right) d \overrightarrow{\mathrm{r}}_{1}^{\prime} \mathrm{d} \vec{\xi}^{\prime}
$$

gives the asymptotic behavior in the channel $\alpha \mathrm{m}$. Thus, for large values of $\mathbf{r}_{0}, \mathrm{U}_{\mathrm{m}}\left(\overrightarrow{\mathrm{r}}_{0}\right)$ becomes equal to the $\mathrm{m}^{\text {th }}$ coefficient in equation (1), if $\alpha \mathrm{m}$ is an open channel, or vanishes if the channel is closed. In the same way, the asymptotic behavior in channel $\beta n$ is given by $\Pi_{\beta n} \Psi=V_{n}(\chi \psi)_{n}$, where $V_{n}(\vec{R})=\left\langle\vec{R},(\chi \psi)_{n} \mid \Psi\right\rangle$.

Any two channel subspaces $\$_{\alpha \mathrm{m}}$ and $\$_{\alpha \mathrm{m}}$, are orthogonal, if $\mathrm{m} \neq \mathrm{m}^{\prime}$, simply because $\left(\phi_{\mathrm{m}} \mid \phi_{\mathrm{m}^{\prime}}\right)=0$. Let $\mathrm{A}$ denote any selected set of $\alpha$ channels and $\mathrm{B}$ denote any selected set of $\beta$ channels. Then, the channel subspaces of the set $A$ together span a subspace $\mathcal{F}_{\mathrm{A}}$, which is the direct sum

$$
\mathfrak{S}_{\mathrm{A}}=\mathfrak{S}_{\alpha \mathrm{m}} \oplus \mathfrak{S}_{\alpha \mathrm{m}^{\prime}} \oplus \ldots \oplus \mathfrak{S}_{\alpha \mathrm{m}^{\prime \prime}} \mathrm{m}, \mathrm{m}^{\prime}, \ldots, \mathrm{m}^{\prime \prime} \subset \mathrm{A}
$$

and has the projection operator

$$
\begin{gathered}
\Pi_{\mathrm{A}}=\sum_{\mathrm{m} \subset \mathrm{A}} \Pi_{\alpha \mathrm{m}} \\
\Pi_{\alpha \mathrm{m}{ }_{\alpha \mathrm{m}^{\prime}}=\delta_{\mathrm{mm}^{\prime}} \Pi_{\alpha \mathrm{m}}}
\end{gathered}
$$

The same considerations apply to the channel subspaces $\mathfrak{S}_{\beta \mathrm{n}}$. The overlap integral

$$
\mathrm{g}_{\mathrm{jm}}\left(\overrightarrow{\mathrm{r}}_{1}\right)=\left(\overrightarrow{\mathrm{r}}_{1}, \psi_{\mathrm{j}} \mid \phi_{\mathrm{m}}\right)=\int \mathrm{d} \vec{\xi}^{\prime} \psi_{\mathrm{j}} *\left(\vec{\xi}^{\prime}\right) \phi_{\mathrm{m}}\left(\overrightarrow{\mathrm{r}}_{1}, \vec{\xi}^{\prime}\right)
$$

gives the component of $\phi_{\mathrm{m}}$ on $\psi_{\mathrm{j}}$.

A projection operator $\mathbf{P}$ that selects all the open channels at the given energy has the property that $P \Psi$ yields the same asymptotic behavior in all channels as does $\Psi$ itself. The projective space $\mathscr{P}$ of $\mathrm{P}$ is the open-channel subspace, and its complement $\mathscr{Q}$, into which $\mathbf{Q}=1$ - $\mathbf{P}$ projects, is the closed-channel subspace. In a reaction, resonance behavior may occur because of the formation of a relatively long-lived compound state of the total system or a long-lived state of an intermediate fragment, when channels with more than two particles are open. Such a quasi-stationary state is described by the vector $Q \Psi$. In configuration space, the representative of $Q \Psi$ becomes vanishing outside regions in which fragments of an open channel are interacting.

Assume that no portion of the channel subspaces $5_{\alpha \mathrm{m}}$ or $\mathfrak{S}_{\beta \mathrm{n}}$ belonging to open 
channels is required in the description of a true compound state and, hence, that $\mathscr{P}$ can be chosen to contain these subspaces (ref. 7). In the case of a nucleon-nucleus collision, the long-lived compound state of the total system can be visualized as being formed by a succession of particle interactions. The process starts when the incident nucleon interacts with the target nucleons in their ground state of motion. The true compound nucleus state is reached through sequences of interactions in which the motion of the compound system becomes progressively more complicated. Beyond some stage in the process, the system is described by the part $Q \Psi$. The class of open-channel projection operators which will be employed is based on the assumption that, in the expansion of $\Psi$ in a complete set such as the $\phi_{\mathrm{m}}$, no open channel $\phi_{\mathrm{m}}$ is required in the description of the compound system after the excitation sequences have attained a certain degree of complexity. Then, there exist open-channel projectors $P$ belonging to the class, since (1-P) $\Psi$ can describe the later stages of the compound system somewhere (depending on the exact choice of $P$ ) beyond this point.

At the given energy, let the set of all open $\alpha$ channels be $A^{0}=\left(0,1,2, \ldots ., M^{\circ}\right)$ and the set of all open $\beta$ channels be $\mathrm{B}^{\mathrm{O}}=\left(0,1,2, \ldots ., \mathrm{N}^{0}\right)$. If $\mathscr{P}$ contains the subspace $S_{A}$, then $P$ may be written in the form

$$
\left.\begin{array}{c}
P=\Pi_{A^{o}}+\Pi_{O} \\
\Pi_{\mathrm{A}}{ }^{\Pi_{O}}=0
\end{array}\right\}
$$

where $\Pi_{\mathrm{O}}$ projects onto the largest subspace of $\mathscr{P}$ that is orthogonal to $\mathfrak{A}^{0^{*}}$ Similarly, if $\mathscr{P}$ contains $\mathfrak{S}_{\mathrm{B}^{\circ}}$, then $\mathbf{P}$ has the form

$$
\left.\begin{array}{c}
P=\Pi_{B^{\circ}}+\Pi_{O^{\prime}} \\
\Pi_{B^{O^{O}} \Pi_{O^{\prime}}=0}
\end{array}\right\}
$$

where $\Pi_{\mathrm{O}}$, projects onto the largest subspace of $\mathscr{P}$ which is orthogonal to $\oiint_{\mathrm{B}^{\circ}}$. Every open-channel subspace $\mathscr{P}$ to be discussed herein contains the channel subspaces in their entirety and has a projector that can be expressed as in equations (8) and (9). The channel subspaces themselves are defined differently in the section RELATED PROJECTION OPERATORS, but forms exactly analogous to equations (8) and (9) will hold.

The open-channel subspaces $\mathscr{P}$ of the class considered herein are special cases of projective spaces that contain the selected subspaces $\sqrt{\mathrm{A}}_{\mathrm{A}}$ and $\widehat{\Phi}_{\mathrm{B}}$. The construction of 
projection operators for the more general case is treated. No complications arise in the more general case. Such projection operators $\Pi$ satisfy the subsidiary conditions

$$
\begin{array}{cc}
\Pi_{\alpha \mathrm{m}}(1-\Pi)=0 & \mathrm{~m} \subset \mathrm{A} \\
\Pi_{\beta \mathrm{n}}(1, \Pi)=0 & \mathrm{n} \subset \mathrm{B}
\end{array}
$$

The projector $\Pi$ qualifies as an open-channel projection operator of the class given by equations (8) and (9) when the sets $A$ and $B$ contain, respectively, $\mathrm{A}^{\circ}$ and $\mathrm{B}^{\circ}$.

If no compound states occur in the reaction, there is no restriction whatever on the extent of the subspace $\mathscr{P}$ in the Hilbert space of the problem. In the effective Hamiltonian for $P \Psi$, the part that contains the rapid energy dependence associated with any resonance effect will have, in this case, a smooth energy variation. (The effective Hamiltonian is given by equation (148) in the last section of this report, and the resonance part is the second term on the right.) In fact, $\mathscr{P}$ can be taken to be the entire space, so that $\mathbf{P}$ is then the unit operator. Consider the scattering problem defined by a phenomenological potential model, to which are ascribed some number of internal states that involve some degrees of freedom and correspond to certain excitations of a many-body system. Such a model is employed to reproduce the part of the direct scattering that involves these excitations in the real many-body case. The Hilbert space in the model problem corresponds to a subspace $\mathscr{P}_{\mathbf{M}}$ of an open-channel subspace $\mathscr{P}$ in the full manybody problem. The projector $\Pi_{\mathbf{M}}$ onto $\mathscr{P}_{\mathbf{M}}$ is the projection operator for a selected set of open channels in the full problem. An exact generalized potential, which corresponds to the model, can be expressed uniquely in terms of $\Pi_{M}$ and $P$. The scattering eigenstate $\theta^{(+)}$given by the potential derived from $\Pi_{\mathbf{M}}$ satisfies $\Pi_{\mathbf{M}^{\theta^{(+)}}=\theta^{(+)}}$. This exact potential is defined so that $\theta^{(+)}$yields transition amplitudes for the selected set of channels that are identical to the corresponding energy -averaged transition amplitudes of the system. The potential will have imaginary parts to simulate the effects of the excluded open channels, as well as the energy-averaged effects of the processes that proceed through the compound states.

If now the internal states appearing in $\Pi_{\mathbf{M}}$ are replaced by the corresponding approximate states employed in the model, the resulting projector $\bar{\Pi}_{\mathbf{M}}$ acts like the unit operator on the scattering state $\bar{\theta}^{(+)}$given by the model. The projector can be employed in the model calculation, however, as a formal device for obtaining the coupled equations that describe the reaction. The conditions (eqs. (10) and (11)) can then be regarded sim ply as properties of the projector that are useful in constructing it. There are, of course, projection operators other than $\Pi_{M}$, that yield the correct asymptotic behavior in the selected channels. An operator $\Pi_{S}$ having this property could be a projection into 
a subspace of $\mathscr{P}_{\mathbf{M}}$. Such a projector may belong to the same class as $\Pi_{\mathbf{M}}$, or may be entirely different from the type of projectors discussed here. In any event, $\Pi_{S}$ enable us to write

$$
\begin{gathered}
\Pi_{M}=\Pi_{S}+Q_{S} \\
\Pi_{S} Q_{S}=0
\end{gathered}
$$

where $\Pi_{S}$ does not act like the unit operator on $\theta^{(+)}$. The corresponding properties hold for $\bar{\Pi}_{M}$ and $\bar{\Pi}_{S}$, the counterparts in the model problem. In the computation of the scattering solution $\bar{\theta}^{(+)}$, the resolution into two orthogonal parts might be' useful, since the part $\overline{\mathrm{Q}}_{\mathbf{S}} \bar{\theta}^{(+)}$is limited to the interior (interaction) region of the configuration space of the model.

In a phenomenological calculation, the following view can be adopted: Employ the suitable projector $\bar{\Pi}_{M}$ for the problem. The model potential that should be used ultimately is the one that approximates most closely the exact potential derived from the associated $\Pi_{\mathbf{M}}$. In the calculations, $\bar{\Pi}_{\mathbf{M}}$ is always taken as the unit operator in the problem, and the potential is adjusted so that the scattering given by the model agrees best with experiment.

\section{Transformation Functions}

For every pair of channels, $\alpha \mathrm{m}$ and $\beta \mathrm{n}$, a transformation function can be defined. The indices $m$ and $n$ will be suppressed for the present to discuss, for a given pair of channels, the transformation function

$$
\mathrm{K}_{1}\left(\overrightarrow{\mathrm{r}}_{0}, \overrightarrow{\mathrm{R}}\right)=\left\langle\overrightarrow{\mathrm{r}}_{0}, \phi \mid \overrightarrow{\mathrm{R}}, \chi \psi\right\rangle
$$

and its adjoint

$$
\mathrm{K}_{1}^{\dagger}\left(\overrightarrow{\mathrm{R}}, \overrightarrow{\mathrm{r}}_{0}\right)=\left\langle\overrightarrow{\mathrm{R}}, \chi \psi \mid \overrightarrow{\mathrm{r}}_{0}, \phi\right\rangle=\mathrm{K}_{1} *\left(\overrightarrow{\mathrm{r}}_{0}, \overrightarrow{\mathrm{R}}\right)
$$

When a vector in $\vec{R}$ space is operated upon by $K_{1}$, the result is a vector in $\vec{r}_{0}$ space, and conversely for $K_{1}^{\dagger}$. The evaluation of $K_{1}$ follows easily: 


$$
\begin{aligned}
\mathbf{K}_{1}\left(\overrightarrow{\mathrm{r}}_{0}, \overrightarrow{\mathrm{R}}\right) & =\int\left\langle\overrightarrow{\mathrm{r}}_{0}, \phi \mid \overrightarrow{\mathrm{r}}_{0}^{\prime}, \overrightarrow{\mathrm{r}}_{1}^{\prime}, \vec{\xi}^{\prime}\right\rangle \mathrm{d} \tau^{\prime}\left\langle\overrightarrow{\mathrm{R}}^{\prime}, \overrightarrow{\mathrm{r}}^{\prime}, \vec{\xi}^{\prime} \mid \overrightarrow{\mathrm{R}}, \chi \psi\right\rangle \\
& =\int \delta\left(\overrightarrow{\mathrm{r}}_{0}-\overrightarrow{\mathrm{r}}_{0^{\prime}}\right) \phi^{*}\left(\overrightarrow{\mathrm{r}}_{1}^{\prime}, \vec{\xi}^{\prime}\right) \mathrm{d} \tau^{\prime} \delta\left(\overrightarrow{\mathrm{R}}^{\prime}-\overrightarrow{\mathrm{R}}\right) \chi\left(\overrightarrow{\mathrm{r}^{\prime}}\right) \psi\left(\vec{\xi}^{\prime}\right)
\end{aligned}
$$

In the volume element, the fact now used is that $d \vec{r}_{1}=8 \mathrm{dR}$ when $\vec{r}_{0}$ is held constant. (This result is equivalent, of course, to the Jacobian of the transformation from $\left(\vec{r}_{0}, \vec{r}_{1}\right)$ to $\left(\vec{r}_{0}, \vec{R}\right)$ having the value $\left[m_{p} /\left(m_{n}+m_{p}\right)\right]^{3}=1 / 8$.) The following equation is finally obtained

$$
\mathrm{K}_{1}\left(\overrightarrow{\mathrm{r}}_{0}, \overrightarrow{\mathrm{R}}\right)=8 \mathrm{~g} *\left(\overrightarrow{\mathrm{r}}_{1}\right) \chi(\overrightarrow{\mathrm{r}})=8 \mathrm{~g} *\left(2 \overrightarrow{\mathrm{R}}-\overrightarrow{\mathrm{r}}_{0}\right) \chi\left(2 \overrightarrow{\mathrm{r}}_{0}-2 \overrightarrow{\mathrm{R}}\right)
$$

where $\mathrm{g}\left(\overrightarrow{\mathrm{r}}_{1}\right)$ has been defined by equation (7). From the transform $\mathrm{K}_{1}$, two iterates can be constructed, namely, $\mathrm{K}=\mathrm{K}_{1} \mathrm{~K}_{1}^{\dagger}$ and $\overline{\mathrm{K}}=\mathrm{K}_{1}^{\dagger} \mathrm{K}_{1}$. These iterates are nonnega tive, Hermitian operators in $\vec{r}_{0}$ space and $\vec{R}$ space, respectively. The operators $K$ and $\overline{\mathbf{K}}$ are represented by the expressions

$$
\begin{aligned}
\mathrm{K}\left(\overrightarrow{\mathrm{r}}_{0}, \overrightarrow{\mathrm{r}}_{0}^{\prime}\right) & =\int \mathrm{K}_{1}\left(\overrightarrow{\mathrm{r}}_{0}, \overrightarrow{\mathrm{R}}^{\prime}\right) \mathrm{d} \overrightarrow{\mathrm{R}}^{\prime} \mathrm{K}_{1}^{\dagger}\left(\overrightarrow{\mathrm{R}}^{\prime}, \overrightarrow{\mathrm{r}}_{0}^{\prime}\right) \\
& =\left\langle\overrightarrow{\mathrm{r}}_{0}, \phi\left|\Pi_{\beta}\right| \overrightarrow{\mathrm{r}}_{0}^{\prime}, \phi\right\rangle
\end{aligned}
$$

and

$$
\overline{\mathrm{K}}\left(\overrightarrow{\mathrm{R}}, \overrightarrow{\mathrm{R}}^{\prime}\right)=\left\langle\overrightarrow{\mathrm{R}}, \chi \psi\left|\Pi_{\alpha}\right| \overrightarrow{\mathrm{R}}^{\prime}, \chi \psi\right\rangle
$$

These operators occur in such projector products as

$$
\begin{aligned}
\Pi_{\alpha} \Pi_{\beta} & =\int d{\overrightarrow{r_{0}^{\prime}}}_{0}\left|\vec{r}_{0}^{\prime}, \phi\right\rangle \mathrm{K}_{1}\left(\overrightarrow{\mathrm{r}}_{0}^{\prime}, \overrightarrow{\mathrm{R}}^{\prime}\right)\left\langle\overrightarrow{\mathrm{R}}^{\prime}, \chi \psi\right| \mathrm{d} \overrightarrow{\mathrm{R}}^{\prime} \\
& \left.=\mathrm{K}_{1} \mid \phi\right)(\chi \psi \mid \\
\Pi_{\alpha} \Pi_{\beta} \Pi_{\alpha} & =\int \mathrm{d}_{\mathbf{r}_{0}^{\prime}}\left|\overrightarrow{\mathrm{r}}_{0}^{\prime}, \phi\right\rangle \mathrm{K}\left(\overrightarrow{\mathrm{r}}_{0}^{\prime}, \overrightarrow{\mathrm{r}}_{0}^{\prime \prime}\right)\left\langle\overrightarrow{\mathrm{r}}_{0}^{\prime \prime}, \phi\right| \mathrm{d} \overrightarrow{\mathrm{r}}_{0}^{\prime \prime} \\
& =\mathrm{K} \mid \phi)(\phi \mid
\end{aligned}
$$




$$
\begin{aligned}
\Pi_{\beta} \Pi_{\alpha} \Pi_{\beta} & =\int \mathrm{d} \overrightarrow{\mathrm{R}}^{\prime}\left|\overrightarrow{\mathrm{R}}^{\prime}, \chi \psi\right\rangle \overline{\mathrm{K}}\left(\overrightarrow{\mathrm{R}}^{\prime}, \overrightarrow{\mathrm{R}}^{\prime \prime}\right)\left\langle\overrightarrow{\mathrm{R}}^{\prime \prime}, \chi \psi\right| \mathrm{d} \overrightarrow{\mathrm{R}}^{\prime \prime} \\
& =\overline{\mathrm{K}} \mid \chi \psi)(\chi \psi \mid
\end{aligned}
$$

In equations (13) to (15), the operators are exhibited in separable form with the transform for either $\vec{r}_{0}$ space or $\vec{R}$ space factored out in each case. The separable property of the projectors and their products ensues from the character of the channel subspaces, which they transform. Each channel subspace itself is a linear manifold whose vectors all have a certain separable form.

The operation of $\Pi_{\alpha}$ and $\Pi_{\beta}$ can be summarized conveniently in the following set of rules:

$$
\begin{gathered}
\Pi_{\alpha}(\rho \varphi)=\rho \phi \\
\Pi_{\alpha}(\sigma \chi \psi)=\left(\mathrm{K}_{1} \sigma\right) \phi \\
\Pi_{\beta}(\rho \phi)=\left(\mathrm{K}_{1}^{\dagger} \rho\right) \chi \psi \\
\Pi_{\beta}(\sigma \chi \psi)=\sigma \chi \psi
\end{gathered}
$$

In the next section, it is shown that constructing a suitable open channel projector from $\Pi_{\alpha}$ and $\Pi_{\beta}$ requires the inverse operators

$$
\left.\begin{array}{l}
\mathrm{L}=\left(1_{\mathrm{r}_{0}}-\mathrm{K}\right)^{-1} \\
\overline{\mathrm{L}}=\left(1_{\mathrm{R}}-\overline{\mathrm{K}}\right)^{-1}
\end{array}\right\}
$$

But $\mathrm{K}_{1}$ is not, in general, represented by a separable function of the variables $\left(\vec{r}_{0}, \vec{R}\right)$. As a result, $K$ and $\bar{K}$ cannot be obtained in closed form. The desired projector can be expressed in terms of the eigenfunctions of $\mathrm{K}$ and $\overline{\mathrm{K}}$, and this form serves to show its general structure. The eigenfunction expansion, however, does not represent a practical method of evaluation because it requires the difficult preliminary step of computing the eigenfunctions.

The operators $K$ and $\bar{K}$ have a common eigenvalue spectrum. If $\lambda^{2} \mathrm{Ku}_{\lambda}=\mathrm{u}_{\lambda}$, $\mathrm{K}_{1}^{\dagger} \mathrm{u}_{\lambda}$ is an eigenfunction of $\overline{\mathbf{K}}$ with the same eigenvalue. Let $v_{\lambda}$ denote the suitably normalized eigenfunction of $\overline{\mathbf{K}}$ (with the square integrable eigenfunctions normalized to unit magnitude). The eigenfunctions $u_{\lambda}$ and $v_{\lambda}$ satisfy a pair of coupled equations 


$$
\left.\begin{array}{c}
\lambda \mathrm{K}_{1}{ }^{\dagger} \mathrm{u}_{\lambda}=-\mathrm{v}_{\lambda} \\
\lambda \mathrm{K}_{1} \mathrm{v}_{\lambda}=-\mathrm{u}_{\lambda}
\end{array}\right\}
$$

where $\lambda$ is real. Because $\phi, \chi$, and $\psi$ are bound states, the kernels $K$ and $\overline{\mathrm{K}}$ vanish exponentially when either of their arguments becomes large. The kernels are consequently square integrable and, hence, bounded (normalizable) kernels. The eigenvalue spectrum is discrete in any finite interval, and each discrete eigenvalue has finite multiplicity.

There is no eigenvalue equal to zero. The expansions of $\mathrm{K}$ and $\overline{\mathrm{K}}$, in their respective sets of orthonormal eigenfunctions, converge. The kernel $\mathrm{K}_{1}$ has the bilinear expansion $\left.K_{1}=\Sigma_{\lambda}(1 / \lambda) \mid u_{\lambda}\right)\left(v_{\lambda} \mid\right.$, whereas $K$ and $L$ have the expansions

$$
\begin{gathered}
\left.\mathrm{K}=\Sigma_{\lambda}\left(1 / \lambda^{2}\right) \mid \mathrm{u}_{\lambda}\right)\left(\mathrm{u}_{\lambda} \mid\right. \\
\left.\mathrm{L}=1_{\mathbf{r}_{0}}+\Sigma_{\lambda}\left(\lambda^{2}-1\right)^{-1} \mid \mathrm{u}_{\lambda}\right)\left(\mathrm{u}_{\lambda} \mid\right.
\end{gathered}
$$

The set of $u_{\lambda}$ having finite eigenvalues is not complete. The additional orthonormal functions that must be added to complete the set can be considered as eigenfunctions belonging to the eigenvalue infinity. From the asymptotic behavior of $\mathrm{K}$, it is seen that for finite $\lambda, u_{\lambda}$ vanishes when $\mathrm{r}_{0}$ becomes large. The additional eigenfunctions do not enter into the expansion (eq. (19)) of $K$ or of its resolvent kernel (the second term on the right of eq. (20)). All the summations just shown have no contribution from these additional eigenfunctions. However, when the expression $\left.L=\Sigma \lambda^{2}\left(\lambda^{2}-1\right)^{-1} \mid u_{\lambda}\right)\left(u_{\lambda} \mid\right.$ is written, it is understood that the sum extends over the complete set. An exactly parallel discussion holds for the expansion of $\overline{\mathrm{K}}$ and $\overline{\mathrm{L}}$ in the $\mathrm{v}_{\lambda}$.

\section{THE PROJECTOR $\Pi_{\alpha+\beta}$}

Take any pair of channel subspaces $\mathfrak{S}_{\alpha \mathrm{m}}$ and $\mathfrak{5}_{\beta \mathrm{n}}$. As an example of a linear space that contains these two subspaces, what might be called the minimal one is first discussed, namely, the space that is spanned by all the vectors of $5_{\alpha m}$ and $\mathfrak{S}_{\beta \mathrm{n}}$ combined. The indices $\mathrm{m}$ and $\mathrm{n}$ may be suppressed for convenience, and the desired projective space designated by the notation

$$
\mathfrak{S}_{\alpha+\beta}=\left\{\mathfrak{s}_{\alpha}, \mathfrak{s}_{\beta}\right\}
$$


When $\mathrm{m}=\mathrm{n}=0$, the projection operator for $\varsigma_{\alpha+\beta}$ qualifies as an open channel projector in the two-channel case, where only the lowest internal energy state in each channel is open. The space defined by equation (21) can also be prescribed by the formulas

$$
\begin{aligned}
& \mathfrak{S}_{\alpha+\beta}=\mathfrak{S}_{\alpha} \oplus \mathfrak{s}_{\beta p \alpha} \\
& \mathfrak{S}_{\alpha+\beta}=\mathfrak{S}_{\beta} \oplus \mathfrak{S}_{\alpha \mathrm{p} \beta}
\end{aligned}
$$

Here, $\mathfrak{S}_{\beta \mathrm{p} \alpha}$ is the largest subspace of $\mathfrak{S}_{\beta}$ that is orthogonal to $\mathfrak{S}_{\alpha}$ and can be viewed as the space spanned by the components orthogonal to $\S_{\alpha}$ of all the vectors in $\aleph_{\beta}$, or equivalently, of all the vectors belonging to a basis set for $5_{\beta}$. To the equivalent direct sums (eqs. (22) and (23)), there correspond equivalent forms for the projector onto the subspace, namely,

$$
\left.\begin{array}{c}
\Pi_{\alpha+\beta}=\Pi_{\alpha}+\Pi_{\beta \mathrm{p} \alpha}=\Pi_{\beta}+\Pi_{\alpha \mathrm{p} \beta} \\
\Pi_{\alpha} \Pi_{\beta \mathrm{p} \alpha}=0 \\
\Pi_{\beta} \Pi_{\alpha \mathrm{p} \beta}=0
\end{array}\right\}
$$

Let a set of basis vectors for $5_{\beta}$ be $\mathrm{y}_{\lambda} \equiv \sigma_{\lambda} \chi \psi$, where the functions $\sigma_{\lambda}(\overrightarrow{\mathrm{R}})$ form a complete orthonormal set for functions of $\vec{R}$. Then $\mathfrak{S}_{\beta p \alpha}$ is spanned by the vectors $\left(1-\Pi_{\alpha}\right) \mathrm{y}_{\lambda}$, which have the scalar products

$$
\begin{aligned}
\left\langle\left(1-\Pi_{\alpha}\right) \mathrm{y}_{\lambda} \mid\left(1-\Pi_{\alpha^{\prime}}\right) \mathrm{y}_{\lambda^{\prime}}\right\rangle & =\left\langle\mathrm{y}_{\lambda} \mid \mathrm{y}_{\lambda^{\prime}}\right\rangle-\left\langle\Pi_{\alpha^{\prime}} \mathrm{y}_{\lambda} \mid \Pi_{\alpha^{\prime}} y_{\lambda^{\prime}}\right\rangle \\
& =\delta_{\lambda \lambda^{\prime}}-\left(\mathrm{K}_{1} \sigma_{\lambda} \mid \mathrm{K}_{1} \sigma_{\lambda^{\prime}}\right)
\end{aligned}
$$

The operational rule (eq. (16)) was used in the last step. If now the choice $\sigma_{\lambda}=v_{\lambda}$ is made, then with the aid of equation (18), the vectors $\left(1-\Pi_{\alpha}\right) y_{\lambda}$ will be orthogonal to each other and $\lambda^{2} \geq 1$. An orthonormal basis set for $\mathfrak{S}_{\beta \mathrm{p} \alpha}$ is given by the vectors

$$
\begin{aligned}
\bar{y}_{\lambda} & =M_{\lambda}\left(1-\Pi_{\alpha}\right) v_{\lambda} \chi \psi \\
& =M_{\lambda}\left(v_{\lambda} \chi \psi+\lambda^{-1} u_{\lambda} \phi\right)
\end{aligned}
$$


where the normalization constant is $M_{\lambda}=\left(1-\lambda^{-2}\right)^{-1 / 2}$. In the same way, an orthonormal basis for $\$_{\alpha p \beta}$ is given by the set

$$
\begin{aligned}
\overline{\mathbf{x}}_{\lambda} & =\mathrm{M}_{\lambda}\left(1-\Pi_{\beta}\right) \mathrm{u}_{\lambda} \phi \\
& =\mathbf{M}_{\lambda}\left(\mathrm{u}_{\lambda} \phi+\lambda^{-1} \mathrm{v}_{\lambda} \chi \psi\right)
\end{aligned}
$$

The projector $\Pi_{\beta \mathrm{p} \alpha}$ can be written with the help of equation (25) as

$$
\begin{gathered}
\Pi_{\beta \mathrm{p} \alpha}=\Sigma_{\lambda}\left|\overline{\mathrm{y}}_{\lambda}\right\rangle\left\langle\overline{\mathrm{y}}_{\lambda}\right| \\
\left.\Pi_{\beta \mathrm{p} \alpha}=\left(\mathrm{L}-\mathbf{1}_{\mathrm{r}_{0}}\right) \mid \phi\right)\left(\phi\left|-\mathbf{L} \mathrm{K}_{1}\right| \phi\right)(\chi \psi|+\overline{\mathrm{L}}| \chi \psi)\left(\chi \psi\left|-\overline{\mathrm{L}} \mathrm{K}_{1}^{\dagger}\right| \chi \psi\right)(\phi \mid
\end{gathered}
$$

and a corresponding form for $\Pi_{\alpha p \beta}$ follows from the basis vectors (eq. (26)). Note that, if the eigenvalues $\lambda^{2}=1$ occur, the corresponding vectors $\left(1-\Pi_{\alpha}\right) \mathrm{y}_{1}$ are null vectors and, therefore, do not contribute to the sum in equation (27).

The projection operator $\Pi$ given by Mittleman (ref. 2) is now discussed. This operator can be written as

$$
\Pi=\mathrm{R}_{\alpha}+\mathrm{R}_{\beta}
$$

Then, $\Pi$ is defined by the following two properties: (1) for any state vector $\Psi, \Pi \Psi$ has the form

$$
\left.\begin{array}{l}
\mathrm{R}_{\alpha} \Psi=\mathrm{u} \phi \\
\mathrm{R}_{\beta} \Psi=\mathrm{v} \chi \psi
\end{array}\right\}
$$

and (2) $\Pi$ satisfies equations (10) and (11). Both conditions (1) and (2) are required to specify the operator uniquely. The form (eq. (30)) is desirable for computational purposes. Coupled equations for $u\left(\vec{r}_{0}\right)$ and $v(\vec{R})$, each functions of a single coordinate, describe the relative inotion in the $\alpha$ and $\beta$ channels in terms of the natural coordinates for each channel. At a large magnitude of its argument, each function yields the corresponding transition amplitude directly.

That $\Pi$ is identical to $\Pi_{\alpha+\beta}$ is easily shown and is fairly obvious from the discussion of $\Pi_{\alpha+\beta}$ just given. The projective space of $\Pi$ is limited by condition (1) to 
contain no more than $\mathfrak{S}_{\alpha}$ and $\mathfrak{S}_{\beta}$, and by condition (2) is required to contain them in their entirety. To obtain $R_{\alpha}$ and $R_{\beta}$, equations (10) and (11) are written as

$$
\left.\begin{array}{l}
\Pi_{\alpha}\left(1-\Pi_{\beta}\right)(1-\Pi)=0 \\
\Pi_{\beta}\left(1-\Pi_{\alpha}\right)(1-\Pi)=0
\end{array}\right\}
$$

Employing the property (eq. (30)) and the operational rule (eq. (16)) then gives

$$
\begin{array}{r}
\Pi_{\alpha}\left(1-\Pi_{\beta}\right) \Psi=\Pi_{\alpha}\left(1-\Pi_{\beta}\right) \Pi_{\alpha} \mathrm{u} \phi \\
\Pi_{\beta}\left(1-\Pi_{\alpha}\right) \Psi=\Pi_{\beta}\left(1-\Pi_{\alpha}\right) \Pi_{\beta} \mathrm{v} \chi \psi
\end{array}
$$

It follows that

$$
\left.\begin{array}{l}
\mathrm{R}_{\alpha}=\Pi_{\alpha} \Lambda \Pi_{\alpha}\left(1-\Pi_{\beta}\right) \\
\mathrm{R}_{\beta}=\Pi_{\beta} \Pi_{\beta}\left(1-\Pi_{\alpha}\right)
\end{array}\right\}
$$

where $\Lambda$ and $\bar{\Lambda}$ are Hermitian operators in the subspaces $\varsigma_{\alpha}$ and $\varsigma_{\beta}$, respectively, with the defining properties

$$
\begin{aligned}
& \Pi_{\alpha} \Lambda \Pi_{\alpha}\left(1-\Pi_{\beta}\right) \Pi_{\alpha}=\Pi_{\alpha} \\
& \Pi_{\beta} \bar{\Lambda} \Pi_{\beta}\left(1-\Pi_{\alpha}\right) \Pi_{\beta}=\Pi_{\beta}
\end{aligned}
$$

The transforms

$$
\begin{aligned}
& \mathbf{L}\left(\overrightarrow{\mathbf{r}}_{0}, \overrightarrow{\mathbf{r}}_{0^{\prime}}\right)=\left\langle\overrightarrow{\mathbf{r}}_{0}, \phi|\Lambda| \overrightarrow{\mathbf{r}}_{0^{\prime}}, \phi\right\rangle \\
& \overline{\mathbf{L}}\left(\overrightarrow{\mathbf{R}}, \overrightarrow{\mathbf{R}}^{\prime}\right)=\left\langle\overrightarrow{\mathrm{R}}, \chi \psi|\pi| \overrightarrow{\mathbf{R}}^{\prime}, \chi \psi\right\rangle
\end{aligned}
$$

permit $\Lambda$ and $\bar{\Lambda}$ to be written as 


$$
\left.\begin{array}{l}
\left.\Lambda=\Pi_{\alpha} \Lambda \Pi_{\alpha}=\mathrm{L} \mid \phi\right)(\phi \mid \\
\left.\bar{\Lambda}=\Pi_{\beta} \bar{\Lambda} \Pi_{\beta}=\overline{\mathrm{L}} \mid \chi \psi\right)(\chi \psi \mid
\end{array}\right\}
$$

Inserting these expressions for $\Lambda$ and $\bar{\Lambda}$ into equations (33) and (34), along with equations (3), (5), (14), and (15), shows that $L$ and $\bar{L}$ are the inverse operators given by equation (17). The forms (eq. (35)) permit $R_{\alpha}$ and $R_{\beta}$ to be written as

$$
\begin{gathered}
\left.\mathrm{R}_{\alpha}=\mathrm{L} \mid \phi\right)\left(\phi\left|-\mathrm{LK}_{1}\right| \phi\right)(\chi \psi \mid \\
\left.\mathrm{R}_{\beta}=\overline{\mathrm{L}} \mid \chi \psi\right)\left(\chi \psi\left|-\mathrm{LK}_{1}^{\dagger}\right| \chi \psi\right)(\phi \mid
\end{gathered}
$$

Comparison of equations (36) and (37) with equations (24) and (28) verifies that $\Pi=\Pi_{\alpha+\beta}$. From equations (32) to (34), $\mathrm{R}_{\alpha}^{2}=\mathrm{R}_{\alpha}, \mathrm{R}_{\beta}^{2}=\mathrm{R}_{\beta}$ and $\mathrm{R}_{\alpha} \mathrm{R}_{\beta}=\mathrm{R}_{\beta} \mathrm{R}_{\alpha}=0$. The idempotents $R_{\alpha}$ and $R_{\beta}$ are not Hermitian, however, and hence are not orthogonal projections.

A null vector results when $\left(1-\Pi_{\beta}\right) \Pi_{\alpha}$ operates on a vector in the subspace $\mathfrak{S}_{\alpha \beta}=\mathfrak{S}_{\alpha} \cap \mathfrak{S}_{\beta}$, the intersection of $\mathfrak{S}_{\alpha}$ and $\mathfrak{s}_{\beta}$. The operator relation (eq. (33)) is understood to be valid in the subspace of $5_{\alpha}$, which is orthogonal to $5_{\alpha \beta}$. In geometric language, the problem posed in obtaining $\Lambda$ by means of equation (33) is the following: Consider a unit vector in $\aleph_{\alpha}$ that is orthogonal to $\aleph_{\alpha \beta}$. Subtract from this vector its component in $\mathfrak{s}_{\beta}$ and project the remaining vector back into $5_{\alpha}$. Find the operator $\Lambda$ that reconstructs the original vector from the value of the projection. Similar comments apply to the interpretation of equation (34).

Because $5_{\alpha \beta}$ is a subspace of both $5_{\alpha}$ and $5_{\beta}$, any normalized vector in the space can be written in the dual form

$$
\rho_{\phi}=\sigma \chi \psi
$$

where $\rho\left(\overrightarrow{\mathrm{r}}_{0}\right)$ and $\sigma(\overrightarrow{\mathrm{R}})$ are suitably normalized functions. Operating on the relation (eq. (38)) with $\Pi_{\beta}$ and $\Pi_{\alpha}$ gives, respectively, with the help of equation (16), $\mathrm{K}_{1}^{\dagger} \rho=\sigma$ and $\rho=\mathrm{K}_{1} \sigma$. Comparison with equation (18) shows that a vector (eq. (38)) in $5_{\alpha \beta}$ corresponds to an eigenfunction of $K$ (and to the associated eigenfunction of $\bar{K}$ ) with eigenvalue $\lambda^{2}=1$. The converse holds as well, because $u_{1} \phi$ is invariant under the operation of $\Pi_{\alpha} \Pi_{\beta} \Lambda_{\alpha}$. Excluding $\mathfrak{S}_{\alpha \beta}$ from the domain of validity of equations (33) and (34) is equivalent to the elimination of the terms in the sum (eq. (27)) that correspond to $\lambda^{2}=1$. Actually, the relation (eq. (38)) imposes severe restrictions on the possible functional forms of $\rho\left(\overrightarrow{\mathrm{r}}_{0}\right), \sigma(\overrightarrow{\mathrm{R}}), \mathrm{g}\left(\overrightarrow{\mathrm{r}}_{1}\right)$ and $\chi(\overrightarrow{\mathrm{r}})$. The allowed functional forms 
are derived by $\mathrm{Coz}$ (ref. 3). However, $\mathrm{u}_{1}$ and $\mathrm{v}_{1}$ are given therein with an incorrect sign in the exponential arguments. Taking the scalar product with $\psi$ on both sides of equation (38) yields $\rho \mathrm{g}=\sigma \chi$. The forms allowed by this relation are $\rho \propto \exp \left[-(1 / 4)_{\kappa} \mathrm{r}_{0}^{2}\right]$, $\mathrm{g} \propto \exp \left[-(1 / 4)_{\kappa} \mathrm{r}_{1}^{2}\right], \sigma \propto \exp \left[-(1 / 2)_{\kappa} \mathrm{R}^{2}\right]$, and $\chi \propto \exp \left[-(1 / 8)_{\kappa} \mathrm{r}^{2}\right]$, where $\kappa$ is a constant. Consequently, in most problems $\mathfrak{S}_{\alpha \beta}$ is a null space, and the case $\lambda^{2}=1$ does not occur.

Projectors like $\Pi_{\alpha+\beta}$ do not appear promising for computational applications. When more than two channels are open, the construction of the corresponding projector involves the solution of a set of coupled integral equations. The computing effort required in the preliminary problem becomes as great as that for the scattering problem itself. However, the methods and notation developed in this section carry over practically unchanged when the related projection operators that can be evaluated easily are developed. The equivalence of the two definitions of $\Pi_{\alpha+\beta}$ has been rigorously demonstrated, and either way of specifying the projectors to be discussed hereinafter will be freely used.

\section{RELATED PROJECTION OPERATORS}

\section{Redefined Channel Subspaces}

Suitable projection operators exist, closely related to $\Pi_{\alpha+\beta}$, that do have a simple closed form. These projectors follow as the result of describing one type of channel (e.g., the $\beta$ channels) by a pair of variables that are not the natural ones for these channels. Such a choice of coordinates is equivalent to redefining the corresponding channel subspaces. The approach is suggested by the work of Chen and Mittleman, who have given a projector of the kind desired. The projectors derived herein have a more simple form than theirs and are more convenient for computational applications. The two-channel case is treated first. It is then easy to generalize to any number of channels. The projector of Chen and Mittleman is described within the context of this discussion.

The two-channel case is first formulated with sufficient generality that all the related projection operators that are expressible in closed form can be identified. The two most suitable operators are the result of using a redefined $\alpha$ channel or a redefined $\beta$ channel. Consider now the possible choices of coordinate pairs from the set $\vec{r}_{0}, \vec{r}_{1}, \vec{R}$, and $\vec{r}$. The first two variables constitute the natural pair for the $\alpha$ channels. Other descriptions of the $\alpha$ channels are now to be considered. Retain the internal coordinate $\vec{r}_{1}$, but leave open the choice of the other coordinate, $\vec{\rho}_{1} \neq \vec{r}_{1}$, from the remaining three. Similarly, the natural pair for the $\beta$ channels is $(\vec{R}, \vec{r})$, but now fix only the internal coordinate $\vec{r}$. The other coordinate $\vec{\rho}_{2} \neq \vec{r}$ can be freely chosen from the rest. Let $f_{1}$ and $\mathscr{f}_{2}$ be the Jacobian determinants for the respective transformations $\left(\overrightarrow{\mathrm{r}}_{0}, \overrightarrow{\mathrm{r}}_{1}\right) \rightarrow\left(\vec{\rho}_{1}, \overrightarrow{\mathrm{r}}_{1}\right)$ 
and $(\overrightarrow{\mathrm{R}}, \overrightarrow{\mathrm{r}})-\left(\overrightarrow{\rho_{2}}, \overrightarrow{\mathrm{r}}\right)$. Thus,

$$
\begin{aligned}
& f_{1}=\frac{\partial\left(\vec{\rho}_{1}, \vec{r}_{1}\right)}{\partial\left(\vec{r}_{0}, \vec{r}_{1}\right)} \\
& f_{2}=\frac{\partial\left(\vec{\rho}_{2}, \vec{r}\right)}{\partial(\vec{R}, \vec{r})}
\end{aligned}
$$

The transformations are linear, so that $\mathcal{f}_{1}$ and $\delta_{2}$ are real constants.

The channel subspace $S_{\alpha^{\prime} \mathrm{m}}$ is defined as the space spanned by all states $\mathrm{s} \phi_{\mathrm{m}}$ that can be represented by wave functions of the form $\mathrm{s}\left(\vec{\rho}_{1}\right) \phi_{\mathrm{m}}\left(\overrightarrow{\mathrm{r}}_{1}, \vec{\xi}\right) \equiv\left\langle\vec{\rho}_{1}, \overrightarrow{\mathrm{r}}_{1}, \vec{\xi} \mid \mathrm{s} \phi_{\mathrm{m}}\right\rangle$. Such wave functions are normalized on the volume element $\mathrm{d} \tau_{1}=\mathrm{d} \vec{\rho}_{1} \mathrm{~d} \overrightarrow{\mathrm{r}}_{1} \mathrm{~d} \vec{\xi}=\left|\boldsymbol{\delta}_{1}\right| \mathrm{d} \tau$, where $\mathrm{d} \tau=d \vec{r}_{0} d \vec{r}_{1} d \vec{\xi}=d \vec{R} d \vec{r} d \vec{\xi}$. If the new coordinate basis vectors are required to satisfy the relations

$$
\left|\vec{\rho}_{1}, \overrightarrow{\mathbf{r}}_{1}, \vec{\xi}\right\rangle=\left|\delta_{1}\right|^{-1 / 2}\left|\overrightarrow{\mathbf{r}}_{0}, \overrightarrow{\mathbf{r}}_{1}, \vec{\xi}\right\rangle
$$

and

$$
\left|\vec{\rho}_{2}, \overrightarrow{\mathbf{r}}, \vec{\xi}\right\rangle=\left|\delta_{2}\right|^{-1 / 2}|\overrightarrow{\mathrm{R}}, \overrightarrow{\mathbf{r}}, \vec{\xi}\rangle
$$

all wave functions will be properly normalized.

The projection operator into the subspace ${ }^{5} \alpha^{\prime} \mathrm{m}$ is

$$
\begin{aligned}
\Pi_{\alpha^{\prime} \mathrm{m}} & \left.=1_{\rho_{1}} \mid \phi_{\mathrm{m}}\right)\left(\phi_{\mathrm{m}} \mid\right. \\
& =\int \mathrm{d} \vec{\rho}_{1^{\prime}}\left|\vec{\rho}_{1^{\prime}}, \phi_{\mathrm{m}}\right\rangle\left\langle\vec{\rho}_{1^{\prime}}, \phi_{\mathrm{m}}\right|
\end{aligned}
$$

The channel subspace $\mathfrak{S}_{\beta^{\prime} \text { n }}$ is spanned by all states representable by

$$
\mathrm{t}\left(\vec{\rho}_{2}\right) x_{\mathbf{i}}(\overrightarrow{\mathrm{r}}) \psi_{\mathbf{j}}(\vec{\xi}) \equiv\left\langle\vec{\rho}_{2}, \overrightarrow{\mathrm{r}}, \vec{\xi} \mid \mathrm{t}(\chi \psi)_{\mathbf{n}}\right\rangle
$$

and has the projector

$$
\left.\Pi_{\beta^{\prime} \mathrm{n}}=1_{\rho_{2}} \mid[\chi \psi]_{\mathrm{n}}\right)\left([\chi \psi]_{\mathrm{n}} \mid\right.
$$


For any pair of channels, $\alpha^{\prime} m$ and $\beta^{\prime} n$, the channel transformation function (again suppressing the indices $m$ and $n$ ) is written as

$$
\begin{aligned}
\left\langle\vec{\rho}_{1}, \phi \mid \vec{\rho}_{2}, \chi \psi\right\rangle & =\int\left\langle\vec{\rho}_{1}, \phi \mid \overrightarrow{\mathrm{r}}_{0^{\prime}}, \overrightarrow{\mathrm{r}}_{1^{\prime}}, \vec{\xi}^{\prime}\right\rangle \mathrm{d} \tau^{\prime}\left\langle\overrightarrow{\mathrm{R}}^{\prime}, \overrightarrow{\mathrm{r}}^{\prime}, \vec{\xi}^{\prime} \mid \vec{\rho}_{2}, \chi \psi\right\rangle \\
& =\int\left|\mathcal{f}_{1} \mathcal{\delta}_{2}\right|^{1 / 2}\left\langle\vec{\rho}_{1}, \phi \mid \vec{\rho}_{1}^{\prime}, \overrightarrow{\mathrm{r}}_{1^{\prime}}, \vec{\xi}^{\prime}\right\rangle \mathrm{d} \tau^{\prime}\left\langle\vec{\rho}_{2}^{\prime}, \overrightarrow{\mathrm{r}}^{\prime}, \vec{\xi}^{\prime} \mid \vec{\rho}_{2}, \chi \psi\right\rangle \\
& =\left|\mathcal{f}_{1} \mathcal{f}_{2}\right|^{1 / 2} \int \delta\left(\vec{\rho}_{1}-\vec{\rho}_{1^{\prime}}\right) \mathrm{g} *\left(\overrightarrow{\mathrm{r}}_{1^{\prime}}\right) \mathrm{d} \mathrm{v}^{\prime} \chi\left(\overrightarrow{\mathrm{r}}^{\prime}\right) \delta\left(\vec{\rho}_{2}^{\prime}-\vec{\rho}_{2}\right) .
\end{aligned}
$$

where $d v^{\prime}=d \vec{r}_{0}^{\prime} d \vec{r}_{1}^{\prime}=d \vec{R}^{\prime} d \vec{r}^{\prime}=d \vec{r}^{\prime} d \vec{r}_{1}^{\prime}$. Equation (41) clearly shows that optimum forms for the transformation function result when the coordinates $\vec{\rho}_{1}$ and $\vec{\rho}_{2}$ are taken to be the same variable, designated as $\vec{\rho}$. When the same coordinate is used to describe the relative motion in both channels, the transformation function will be proportional to a delta function in the coordinates, and the integration involved in obtaining the first iterates will be trivial

$$
\left\langle\vec{\rho}, \phi \mid \vec{\rho}^{\prime}, \chi \psi\right\rangle=\left|\mathscr{\delta}_{1} \mathscr{L}_{2}\right|^{1 / 2} \delta\left(\vec{\rho}-\vec{\rho}^{\prime}\right) \int \delta\left(\vec{\rho}-\vec{\rho}^{\prime \prime}\right) \mathrm{g}^{*}\left(\overrightarrow{\mathbf{r}}_{1}^{\prime \prime}\right) \mathrm{dv^{ \prime \prime }} \chi\left(\overrightarrow{\mathbf{r}}^{\prime \prime}\right)
$$

The choice of variables has now been narrowed down to $\vec{\rho}$ being either $\vec{r}_{0}$ or $\vec{R}$.

For the sake of completeness, another possibility, is mentioned, namely, $\vec{\rho}_{1}=\vec{r}$, $\vec{\rho}_{2}=\vec{r}_{1}$, which gives $\left\langle\overrightarrow{\mathrm{r}}, \phi \mid \overrightarrow{\mathrm{r}}_{1}, \chi \psi\right\rangle=\chi(\overrightarrow{\mathrm{r}}) \mathrm{g} *\left(\overrightarrow{\mathrm{r}}_{1}\right)$. Because the kernel is separable in these coordinates, it is easy to evaluate the first iterates and to construct the related projection operator $\Pi_{\alpha^{\prime}+\beta^{\prime}}$. However, a projection into redefined channel subspaces for both channels would not appear to have any application, and this case will not be discussed further.

\section{The Projector $\Pi_{\alpha+\beta^{\prime}}$}

Consider first the case where $\vec{\rho}=\overrightarrow{\mathbf{r}}_{0}$ in equation (42). Then, natural coordinates are employed in the $\alpha$ channel. The channel subspace is $5_{\alpha}$ and $\delta_{1}=1$. When the coordinate pair $\left(\overrightarrow{\mathbf{r}}_{0}, \overrightarrow{\mathbf{r}}\right)$ is used with the $\beta$ channel, however, a redefined channel subspace $S_{\beta^{\prime} n}$ serves to describe the $\beta$ channel. The projector onto $\varsigma_{\beta^{\prime} n}$ is given by equation (40) with $\vec{\rho}_{2}=\vec{r}_{0}$. Thus, 


$$
\begin{aligned}
\Pi_{\beta^{\prime} \mathbf{n}} & \left.=1_{\mathbf{r}_{0}} \mid[\chi \psi]_{\mathrm{n}}\right)(x \psi]_{\mathrm{n}} \mid \\
& =\int \mathrm{d} \overrightarrow{\mathbf{r}}_{0^{\prime}}\left|\overrightarrow{\mathbf{r}}_{0^{\prime}},[\chi \psi]_{\mathrm{n}}\right\rangle\left\langle\overrightarrow{\mathbf{r}}_{0^{\prime}},[\chi \psi]_{\mathrm{n}}\right|
\end{aligned}
$$

The Jacobian $\delta_{2}$ has the value 1 , and equation (42) yields directly the transformation kernel

$$
\begin{aligned}
\mathrm{J}_{1}\left(\overrightarrow{\mathrm{r}}_{0}, \overrightarrow{\mathrm{r}}_{0^{\prime}}\right) & =\left\langle\overrightarrow{\mathrm{r}}_{0}, \phi \mid \overrightarrow{\mathrm{r}}_{0}^{\prime} \chi \psi\right\rangle \\
& =\delta\left(\overrightarrow{\mathrm{r}}_{0}-\overrightarrow{\mathrm{r}}_{0^{\prime}}\right) \mathrm{h}\left(\overrightarrow{\mathbf{r}}_{0}\right)
\end{aligned}
$$

where

$$
\mathrm{h}\left(\overrightarrow{\mathrm{r}}_{0}\right)=\int \mathrm{g} *\left(\overrightarrow{\mathrm{r}}_{1}^{\prime}\right) \times\left(\overrightarrow{\mathrm{r}}_{0}-\overrightarrow{\mathrm{r}}_{1}^{\prime}\right) \mathrm{d \mathbf {r } _ { 1 } ^ { \prime }}
$$

From Schwarz's inequality,

$$
\left|\mathrm{h}\left(\overrightarrow{\mathrm{r}}_{0}\right)\right|^{2}<(\mathrm{g} \mid \mathrm{g})
$$

The possibility of the two sides in equation (46) being equal is excluded by the reasonable assumption that $\chi$, as a function of $\vec{r}_{1}$, is never proportional to $g\left(r_{1}\right)$. Because $g \psi$ is a term that occurs in the expansion of $\phi$ in the complete orthonormal set of states of nucleus $\mathscr{A}-1$, the normalization of the bound states $\phi$ and $\psi$ to unit magnitude implies that

$$
c_{0}^{2} \equiv(g \mid g) \leq 1
$$

The equality sign in equation (47) holds only if $\phi=\mathrm{g} \psi$. The bound state nature of the function $\phi$ (or $g$ ) and $\chi$ shows that

$$
\mathrm{h}\left(\overrightarrow{\mathrm{r}}_{0}\right) \approx 0 \quad \text { when } \quad \mathrm{r}_{0} \gg \mathrm{R}_{\mathrm{A}}+\mathrm{R}_{\mathrm{D}}
$$

The first iterates $\mathrm{J}=\mathrm{J}_{1} \mathrm{~J}_{1}^{\dagger}$ and $\overline{\mathrm{J}}=\mathrm{J}_{1}^{\dagger} \mathrm{J}_{1}$ are easily evaluated and are seen to be the same operator in $\vec{r}_{0}$ space 


$$
\begin{aligned}
& \mathrm{J}\left(\overrightarrow{\mathbf{r}}_{0}, \overrightarrow{\mathbf{r}}_{0}{ }^{\prime}\right)=\int \mathrm{d} \overrightarrow{\mathbf{r}}_{0}{ }^{\prime \prime} \mathrm{J}_{1}\left(\overrightarrow{\mathrm{r}}_{0}, \overrightarrow{\mathbf{r}}_{0}{ }^{\prime \prime}\right) \mathrm{J}_{1}^{\dagger}\left(\overrightarrow{\mathrm{r}}_{0}{ }^{\prime \prime}, \overrightarrow{\mathrm{r}}_{0}{ }^{\prime}\right) \\
& =\left\langle\overrightarrow{\mathbf{r}}_{0}, \phi\left|\Pi_{\beta^{\prime}}\right| \overrightarrow{\mathbf{r}}_{0^{\prime}}, \phi\right\rangle \\
& =\delta\left(\overrightarrow{\mathbf{r}}_{0}-\overrightarrow{\mathbf{r}}_{0}{ }^{\prime}\right)\left|\mathbf{h}\left(\overrightarrow{\mathbf{r}}_{0}\right)\right|^{2} \\
& =\left\langle\overrightarrow{\mathbf{r}}_{0}, \chi \psi\left[\Pi_{\alpha}\left|\overrightarrow{\mathbf{r}}_{0^{\prime}}, \chi \psi\right\rangle=\mathrm{J}\left(\overrightarrow{\mathrm{r}}_{0}, \overrightarrow{\mathrm{r}}_{0^{\prime}}\right)\right.\right.
\end{aligned}
$$

The operation of the Hermitian, nonnegative operators $\mathrm{J}$ and $\overline{\mathrm{J}}$ is merely multiplication by $\left|\mathrm{h}\left(\overrightarrow{\mathrm{r}}_{0}\right)\right|^{2}$. Henceforth, the following simplified notation for operators that are diagonal in the $\overrightarrow{\mathbf{r}}_{0}$ representation will be adopted

$$
\left.\begin{array}{r}
\mathrm{J}_{1}=\mathrm{h}^{1} \mathrm{r}_{0} \\
\mathrm{~J}_{1}^{\dagger}=\mathrm{h}^{*}{ }^{1} \mathrm{r}_{0}
\end{array}\right\}
$$

A normalized eigenstate of $J$ is $\delta_{a}$, where $\left(\vec{r}_{0} \mid \delta_{a}\right)=\delta_{a}\left(\vec{r}_{0}\right)=\delta\left(\vec{r}_{0}-\vec{a}\right)$, with the eigenvalue $|h(\vec{a})|^{2}$. The continuous set $\left\{\delta_{a}\right\}$ is a complete orthonormal basis for $\vec{r}_{0}$ space. In contrast to the kernel $\mathrm{K}$ of equation (12), the kernel $\mathrm{J}$ is not bounded: it has such a simple form, however, that the required inverse operators can be constructed directly, and eigenfunction expansions of the operators need not be resorted to at all.

Operational rules, analogous to those of equation (16), are easily established:

$$
\left.\begin{array}{c}
\Pi_{\alpha^{\prime}}(\rho \phi)=\rho \phi \\
\Pi_{\alpha^{\prime}}(\rho \chi \psi)=\left(\mathrm{J}_{1} \rho\right) \phi \\
\Pi_{\beta^{\prime}}(\rho \phi)=\left(\mathrm{J}_{1}^{\dagger} \rho\right) \phi \\
\Pi_{\beta^{\prime}}(\rho \chi \psi)=\rho \chi \psi
\end{array}\right\}
$$

The subspace $\mathfrak{S}_{\alpha+\beta^{\prime}}$ is the space spanned by all the vectors of $\mathfrak{S}_{\alpha}$ and $\mathfrak{S}_{\beta^{\prime}}$ combined. The projector $\Pi_{\alpha+\beta}$, onto this subspace may be constructed from the expressions 


$$
\begin{aligned}
& \Pi_{\alpha+\beta^{\prime}}=\Pi_{\alpha}+\Pi_{\beta^{\prime} p \alpha} \\
& \Pi_{\alpha+\beta^{\prime}}=\Pi_{\beta^{\prime}}+\Pi_{\alpha p \beta}
\end{aligned}
$$

When the only open channels are the lowest $\alpha$ and $\beta$ ones, then the corresponding $\Pi_{\alpha+\beta^{\prime}}$ can be used as the open-channel projection operator. The form (eq. (52)) shows that this projector is particularly appropriate for the pickup process. The $\alpha$ channel is then the incident one, and $\Pi_{\alpha}$ commutes with the noninteracting Hamiltonian for the $\alpha$ fragments. Hence, among its boundary conditions, $\Pi_{\alpha+\beta}, \Psi$ has the same incident plane wave as $\Psi$.

An orthonormal set spanning $\Phi_{\beta}$, is the continuous set of vectors $y_{a}=\delta_{a} \times \psi$. The component of $\mathrm{y}_{\mathrm{a}}$ orthogonal to $5_{\alpha}$ is proportional to the unit vector

$$
\begin{aligned}
\overline{\mathrm{y}}_{\mathrm{a}} & =\mathrm{n}(\overrightarrow{\mathrm{a}})\left(1-\Pi_{\alpha}\right) \mathrm{y}_{\mathrm{a}} \\
& =\mathrm{n}(\overrightarrow{\mathrm{a}}) \delta_{\mathrm{a}}[\chi \psi-\mathrm{h}(\overrightarrow{\mathrm{a}}) \phi]
\end{aligned}
$$

In the last step, equation (51) and the equation $J_{1} \delta_{a}=h(\vec{a}) \delta_{a}$ were used. From equation (54) and the projector properties of $\Pi_{\alpha}$,

$$
\begin{aligned}
\left\langle\mathrm{y}_{\mathrm{a}} \mid \mathrm{y}_{\mathrm{a}^{\prime}}\right\rangle & =\mathrm{n}(\overrightarrow{\mathrm{a}}) \mathrm{n}\left(\overrightarrow{\mathrm{a}}^{\prime}\right)\left[\left\langle\mathrm{y}_{\mathrm{a}} \mid \mathrm{y}_{\mathrm{a}^{\prime}}\right\rangle-\left\langle\Pi_{\alpha^{\prime}} \mathrm{y}_{\mathrm{a}} \mid \Pi_{\alpha^{\prime}} \mathrm{y}_{\mathrm{a}^{\prime}}\right\rangle\right] \\
& =\mathrm{n}^{2}(\overrightarrow{\mathrm{a}})\left(1-|\mathrm{h}(\overrightarrow{\mathrm{a}})|^{2}\right) \delta\left(\overrightarrow{\mathrm{a}}-\overrightarrow{\mathrm{a}}^{\prime}\right)
\end{aligned}
$$

If the normalization constant is chosen to be $n(\vec{a})=\left(1-|h(a)|^{2}\right)^{-1 / 2}$, the vectors $\bar{y}_{a}$ constitute an orthonormal set. Accordingly,

$$
\begin{aligned}
\Pi_{\beta^{\prime} p \alpha} & =\int \mathrm{da}\left|\bar{y}_{\mathrm{a}}\right\rangle\left\langle\overline{\mathrm{y}}_{\mathrm{a}}\right| \\
& =\left(1-\Pi_{\alpha}\right) \int \mathrm{dan}^{2}(\overrightarrow{\mathrm{a}})\left|\mathrm{y}_{\mathrm{a}}\right\rangle\left\langle\mathrm{y}_{\mathrm{a}}\right|\left(1-\Pi_{\alpha}\right) \\
& \left.=\left(1-\Pi_{\alpha}\right) \mathrm{n}^{2} 1_{\mathrm{r}_{0}} \mid \chi \psi\right)\left(\chi \psi \mid\left(1-\Pi_{\alpha}\right)\right. \\
& =\mathrm{n}^{2} 1_{\mathrm{r}_{0}}\left(1-\Pi_{\alpha}\right) \Pi_{\beta^{\prime}}\left(1-\Pi_{\alpha}\right)
\end{aligned}
$$




$$
\begin{aligned}
\Pi_{\beta^{\prime} p \alpha} & =n^{2} 1_{r_{0}}\left[\Pi_{\beta^{\prime}}\left(1-\Pi_{\alpha}\right)+\Pi_{\alpha^{\prime}} \Pi_{\beta^{\prime}}\left(\Pi_{\alpha}-1\right)\right] \\
& =n^{2} 1_{r_{0}}[\mid \chi \psi)\left(\chi \psi\left|-J_{1}^{\dagger}\right| \chi \psi\right)(\phi|+J| \phi)\left(\phi\left|-J_{1}\right| \phi\right)(\chi \psi \mid]
\end{aligned}
$$

The following relation was required in arriving at equation (55):

$$
\left.\int \mathrm{d} \overrightarrow{\mathrm{an}}^{2}(\overrightarrow{\mathrm{a}}) \mid \delta_{\mathrm{a}}\right)\left(\delta_{\mathrm{a}}\left|=\int \mathrm{d} \overrightarrow{\mathrm{r}}_{0} \mathrm{n}^{2}\left(\overrightarrow{\mathrm{r}}_{0}\right)\right| \overrightarrow{\mathrm{r}}_{0}\right)\left(\overrightarrow{\mathrm{r}}_{0} \mid=\mathrm{n}^{2} 1_{\mathbf{r}_{0}}\right.
$$

The product relations involving $\Pi_{\alpha}$ and $\Pi_{\beta^{\prime}}$, which are the direct analogues of equations (13) and (14), were used in the step to equation (56). If the vector $\omega_{0}$, orthogonal to $\mathfrak{f}_{\alpha}$, is defined as $\omega_{\mathrm{o}}=\mathrm{n}(\chi \psi-\mathrm{h} \phi)$, where it is understood that $\mathrm{n}$ and $\mathrm{nh}$ are vectors in $\vec{r}_{0}$ space, the projector can be written in the concise form $\left.\Pi_{\beta^{\prime} p \alpha}=1_{r_{0}} \mid \omega_{0}\right)\left(\omega_{0} \mid\right.$.

Another expression for $\Pi_{\alpha+\beta}$, corresponding to equations (29) and (30) in the previous case, is

$$
\Pi_{\alpha+\beta^{\prime}}=\widetilde{\mathbf{R}}_{\alpha}+\mathbf{R}_{\beta^{\prime}}
$$

where

$$
\left.\begin{array}{c}
\widetilde{R}_{\alpha} \Psi=\widetilde{F} \phi \\
R_{\beta^{\prime}} \Psi=G_{\chi \psi}
\end{array}\right\}
$$

and $\widetilde{F}$ and $G$ are vectors in $\vec{r}_{0}$ space. The other property, in addition to equation (57) and (58), which determines the projector uniquely can be written

$$
\left.\begin{array}{c}
\Pi_{\alpha}\left(1-\Pi_{\beta^{*}}\right)\left(1-\Pi_{\alpha+\beta^{*}}\right)=0 \\
\Pi_{\beta^{\prime}}\left(1-\Pi_{\alpha^{\prime}}\right)\left(1-\Pi_{\alpha+\beta^{*}}\right)=0
\end{array}\right\}
$$

Combining equations (57) and (58) with equation (59) and using rules (eq. (51)) give

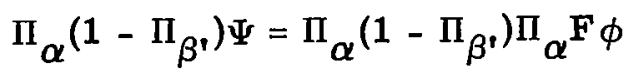

and 


$$
\Pi_{\beta^{\prime}}\left(1-\Pi_{\alpha}\right) \Psi=\Pi_{\beta^{\prime}}\left(1-\Pi_{\alpha}\right) \Pi_{\beta^{\prime}}, G \chi \psi
$$

It immediately follows that

$$
\left.\begin{array}{l}
\tilde{\mathrm{R}}_{\alpha}=\Pi_{\alpha} \Lambda_{\mathrm{O}} \Pi_{\alpha}\left(1-\Pi_{\beta^{\prime}}\right) \\
\mathrm{R}_{\beta^{\prime}}=\Pi_{\beta^{\prime}} \bar{\Lambda}_{\mathrm{O}} \Pi_{\beta^{\prime}}\left(1-\Pi_{\alpha^{\prime}}\right)
\end{array}\right\}
$$

where $\Lambda_{O}$ and $\bar{\Lambda}_{\mathrm{O}}$ are Hermitian operators in the subspaces $\mathfrak{s}_{\alpha}$ and $\mathfrak{s}_{\beta^{\prime}}$, respectively, which are defined by the relations

$$
\left.\begin{array}{c}
\Pi_{\alpha^{\Lambda}} \Lambda_{O^{\prime}}\left(1-\Pi_{\beta^{\prime}}\right) \Pi_{\alpha}=\Pi_{\alpha} \\
\Pi_{\beta^{\prime}} \bar{\Lambda}_{\mathrm{O}^{\prime}} \Pi_{\beta^{\prime}}\left(1-\Pi_{\alpha^{\prime}}\right) \Pi_{\beta^{\prime}}=\Pi_{\beta^{\prime}}
\end{array}\right\}
$$

It is clear that $\widetilde{R}_{\alpha}$ and $R_{\beta^{\prime}}$, are idempotents and satisfy $\widetilde{R}_{\alpha^{\prime}} \mathbf{R}_{\beta^{\prime}}=\mathbf{R}_{\beta^{\prime}} \widetilde{R}_{\alpha}=0$. The separable forms, similar to equation (35),

$$
\begin{gathered}
\left.\Lambda_{\mathrm{O}}=\Pi_{\alpha} \Lambda_{\mathrm{O}} \Pi_{\alpha}=\mathrm{O} \mid \phi\right)(\phi \mid \\
\left.\bar{\Lambda}_{\mathrm{O}}=\Pi_{\beta^{\prime}}, \bar{\Lambda}_{\mathrm{O}} \Pi_{\beta^{\prime}}=\overline{\mathrm{O}} \mid \chi \psi\right)(\chi \psi \mid
\end{gathered}
$$

enable the conversion of equation (61) into the equivalent equations in $\vec{r}_{0}$ space

$$
\begin{aligned}
& O\left(\mathbf{r}_{\mathbf{r}_{0}}-J\right)=\mathbf{1}_{\mathbf{r}_{0}} \\
& \overline{\mathrm{O}}\left(\mathbf{1}_{\mathbf{r}_{0}}-\bar{J}\right)=\mathbf{1}_{\mathbf{r}_{0}}
\end{aligned}
$$

From equation (50), the solutions are simply

$$
\mathrm{O}=\overline{\mathrm{O}}=\mathrm{n}^{2}{ }^{1_{\mathrm{r}_{0}}}
$$

When the solutions (eq. (62)) are inserted into equation (60), the result can be written as 


$$
\left.\begin{array}{c}
\left.\widetilde{\mathbf{R}}_{\alpha}=\mathrm{n}^{2} 1_{\mathbf{r}_{0}} \mid \phi\right)\left(\phi\left|-\mathrm{n}^{2} \mathrm{~h} 1_{\mathbf{r}_{0}}\right| \phi\right)(\chi \psi \mid \\
\left.\mathbf{R}_{\beta^{\prime}}=\mathbf{n}^{2}{ }_{\mathbf{r}_{0}} \mid \chi \psi\right)\left(\chi \psi\left|-\mathbf{n}^{2} \mathbf{h}^{*} 1_{\mathbf{r}_{0}}\right| \chi \psi\right)(\phi \mid
\end{array}\right\}
$$

Comparing equation (63) with equation (56) and using the identity $\left(1+n^{2}|h|^{2}\right)=n^{2}$ verify that the forms (eqs. (52), (53), and (57)) are equivalent.

\section{The Projector $\Pi_{\alpha^{\prime}+\beta}$}

The other choice for the variable in equation (42) is $\vec{\rho}=\vec{R}$. In this case, the coordinate pair for the $\beta$ channel is the natural one. The channel subspace is $5_{\beta j}$ and $\mathcal{f}_{2}=1$. When the pair $\left(\overrightarrow{\mathrm{R}}, \overrightarrow{\mathrm{r}}_{1}\right)$ is taken for the $\alpha$ channel, the redefined channel subspace $5_{\alpha^{\prime} \mathrm{m}}$ has the projector $\left.\Pi_{\alpha^{\prime} \mathrm{m}}=1_{\mathrm{R}} \mid \phi_{\mathrm{m}}\right)\left(\phi_{\mathrm{m}} \mid\right.$. The Jacobian $\delta_{1}$ has the value $1 / 8$, and the transformation function $I_{1}\left(\vec{R}^{\prime}, \vec{R}^{\prime}\right)=\left\langle\vec{R}, \phi \mid \vec{R}^{\prime}, \chi \psi\right\rangle$ is the (diagonal) representation of the operator $I_{1}=(1 / 8) l 1_{R}$, where

$$
\zeta(\mathrm{R})=\int \mathrm{g} *\left(\mathbf{R}-\frac{1}{2} \mathrm{r}^{\prime}\right) \chi\left(\mathrm{r}^{\prime}\right) \mathrm{dr} \mathbf{r}^{\prime}
$$

The first iterates of $I_{1}$ are simply $I=\bar{I}=(1 / 64)|l|^{2} 1_{R}$. These Hermitian operators possess the continuous set of eigenstates $\delta_{b}(\vec{R}) \equiv \delta(\vec{R}-\vec{b})$, which are a complete orthonormal set for $\vec{R}$ space. The vectors $x_{b} \equiv \delta_{b} \phi$ span $\$_{\alpha^{\prime}}$.

The subspace $\mathfrak{5}_{\alpha^{\gamma}+\beta}$, which is spanned by all the vectors of $\mathfrak{5}_{\alpha}$, and $\mathfrak{5}_{\beta}$ combined, has the projector

$$
\begin{gathered}
\Pi_{\alpha^{\prime}+\beta}=\Pi_{\beta}+\Pi_{\alpha^{\prime} \mathrm{p} \beta} \\
\Pi_{\alpha^{\prime}+\beta}=\Pi_{\alpha^{\prime}}+\beta \mathrm{p} \alpha^{\prime} \\
\Pi_{\alpha^{\prime}+\beta}=\mathrm{R}_{\alpha^{\prime}}+\widetilde{\mathrm{R}}_{\beta}
\end{gathered}
$$

where $R_{\alpha}, \Psi=F \phi, \widetilde{R}_{\beta} \Psi=\widetilde{G} \chi \psi$, and $F$ and $\widetilde{G}$ are vectors in $\vec{R}$ space. The form (eq. (64)) shows that open-channel projectors of this type will be useful for the stripping reaction. The vectors 


$$
\begin{aligned}
\bar{x}_{b} & =m(b)\left(1-\Pi_{\beta}\right) x_{b} \\
& =m(b) \delta_{b}[\phi-(1 / 8) l *(b) \chi \psi]
\end{aligned}
$$

with normalization constant $m(\vec{b})=\left[1-(1 / 64)|l(\vec{b})|^{2}\right]^{-1 / 2}$, are an orthonormal set and span $\$_{\alpha \cdot p \beta}$. Hence,

$$
\begin{aligned}
\Pi_{\alpha^{\prime} \mathrm{p} \beta} & =\int \mathrm{d} \overrightarrow{\mathrm{b}}\left|\mathrm{x}_{\mathrm{b}}\right\rangle\left\langle\mathrm{x}_{\mathrm{b}}\right| \\
& =\mathrm{m}^{2} 1_{\mathrm{R}}\left(1-\Pi_{\beta}\right) \Pi_{\alpha^{\prime}}\left(1-\Pi_{\beta}\right) \\
& =\mathrm{m}^{2} 1_{\mathrm{R}}\left[\Pi_{\alpha^{\prime}}\left(1-\Pi_{\beta}\right)+\overline{\mathrm{I}} \mid \phi\right)\left(\phi \mid-\Pi_{\beta^{\prime}}{\alpha^{\prime}}^{\prime}\right] \\
& \left.=1_{\mathrm{R}} \mid \omega_{\mathrm{o}}{ }^{\prime}\right)\left(\omega_{\mathrm{o}}^{\prime} \mid\right.
\end{aligned}
$$

where the vector $\omega_{\mathrm{o}}^{\prime} \equiv \mathrm{m}[\phi-(1 / 8) l * \chi \psi]$ is orthogonal to $\mathfrak{5}_{\beta}$. The form (eq. (65)) can be evaluated by a sequence of steps that directly parallels those which lead from equations (57), (58), and (59) to equation (63). The results are

$$
R_{\alpha^{,}}=\Pi_{\alpha^{,}} \Lambda_{N^{\prime} \Pi^{,}}\left(1-\Pi_{\beta}\right)
$$

and

$$
\widetilde{\mathrm{R}}_{\beta}=\Pi_{\beta} \bar{\Lambda}_{\mathrm{N}} \Pi_{\beta}\left(1-\Pi_{\alpha^{\prime}}\right)
$$

where it is found that

$$
\Lambda_{\mathrm{N}}=\mathrm{m}^{2} 1_{\mathrm{R}} \Pi_{\alpha^{\prime}}
$$

and

$$
\bar{\Lambda}_{\mathrm{N}}=\mathrm{m}^{2} 1_{\mathrm{R}} \Pi_{\beta}
$$

The identity ${ }^{1} R+m^{2} \bar{I}=m^{2} 1_{R}$ is used to verify that equations (64) and (65) are equivalent. 


\section{The Projector $\Pi_{\alpha+c}$}

The subspace $\Phi_{\beta \mathrm{n}}$ is defined with reference to a specific pair of internal states for the $\beta$ fragments $\mathscr{P}$ and $\alpha_{-1}$, as indicated by the notation $(\chi \psi)_{n} \equiv \chi_{i} \psi_{j}$. Consider the set of all channel subspaces obtained with a given state $\psi_{j}$ when $\chi_{i}$ runs through a complete set of states of the nucleus $\mathscr{P}$, including those in the continuum. These spaces are orthogonal to one another, and their direct sum is denoted by $\$_{\text {cj }} \cdot$ Alternatively, $\$_{\text {cj }}$ can be characterized as the subspace spanned by all states that are representable in the form $\xi(\vec{R}, \vec{r}) \psi_{j}(\vec{\xi})$. The projection operator for the subspace spanned by all the vectors of $\Phi_{\alpha \mathrm{m}}$ and $S_{\mathrm{cj}}$ combined can be applied to the pickup or stripping process. This type of projector has been discussed by Feshbach (ref. 1).

The projector onto $\Phi_{\mathrm{cj}}$ can be written in various forms, namely,

$$
\begin{aligned}
\Pi_{c j} & \left.=1_{R^{1}} \mid \psi_{j}\right)\left(\psi_{j}\left|=1_{r_{0}} 1_{r_{1}}\right| \psi_{j}\right)\left(\psi_{j} \mid\right. \\
& =\int\left|\vec{r}_{0^{\prime}}, \vec{r}_{1^{\prime}}, \psi_{j}\right\rangle d \vec{r}_{0^{\prime}} d \vec{r}_{1}{ }^{\prime}\left\langle\vec{r}_{0}, \vec{r}_{1}, \psi_{j}\right|
\end{aligned}
$$

The transformation function $K_{2}$ is easily evaluated

$$
\begin{aligned}
\mathrm{K}_{2}\left(\overrightarrow{\mathbf{r}}_{0} ; \overrightarrow{\mathbf{r}}_{0}, \overrightarrow{\mathbf{r}}_{1} ; \mathrm{m}_{\mathbf{j}}\right) & =\left\langle\overrightarrow{\mathrm{r}}_{0}, \phi_{\mathrm{m}} \mid \overrightarrow{\mathrm{r}}_{0^{\prime}}, \overrightarrow{\mathbf{r}}_{1}^{\prime}, \psi_{\mathrm{j}}\right\rangle \\
& =\delta\left(\overrightarrow{\mathrm{r}}_{0}-\overrightarrow{\mathbf{r}}_{0}^{\prime}\right) \mathrm{g}_{\mathrm{jm}} *\left(\overrightarrow{\mathrm{r}}_{1^{\prime}}\right)
\end{aligned}
$$

Hereinafter in this section, the indices $m$ and $j$ will be supressed. The first iterate $\mathrm{K}^{\prime}=\mathrm{K}_{2} \mathrm{~K}_{2}^{\dagger}$ is an operator in $\overrightarrow{\mathrm{r}}_{0}$ space, while the iterate $\overline{\mathrm{K}}^{\prime}=\mathrm{K}_{2}^{\dagger} \mathrm{K}_{2}$ transforms states of motion of particles 1 and 2 , that is, operates on vectors in $\left(\overrightarrow{\mathbf{r}}_{0} \overrightarrow{\mathbf{r}}_{1}\right)$ space. Evaluating the kernels

$$
\begin{gathered}
\mathbf{K}^{\prime}\left(\overrightarrow{\mathrm{r}}_{0}, \overrightarrow{\mathrm{r}}_{0^{\prime}}\right)=\left\langle\overrightarrow{\mathrm{r}}_{0}, \phi\left|\Pi_{\mathrm{c}}\right| \overrightarrow{\mathrm{r}}_{0}, \phi\right\rangle \\
\overline{\mathbf{K}}^{\prime}\left(\overrightarrow{\mathrm{r}}_{0}, \overrightarrow{\mathbf{r}}_{1} ; \overrightarrow{\mathbf{r}}_{0^{\prime}}, \overrightarrow{\mathbf{r}}_{1^{\prime}}\right)=\left\langle\overrightarrow{\mathbf{r}}_{0}, \overrightarrow{\mathbf{r}}_{1}, \psi\left|\Pi_{\alpha}\right| \overrightarrow{\mathbf{r}}_{0^{\prime}}, \overrightarrow{\mathbf{r}}_{\left.1^{\prime}, \psi\right\rangle}\right.
\end{gathered}
$$

shows that they are the representatives of the operators 


$$
\left.\begin{array}{c}
K^{\prime}=c_{o}^{2}{ }^{1} r_{0} \\
\mid g)\left(g\left|=c_{0}^{2}{ }_{1}\right| w_{0}\right)\left(w_{1} \mid\right.
\end{array}\right\}
$$

where $w_{2}$ is the unit vector $w_{1}=\left(c_{o}\right)^{-1} g$. The eigenstates of $K^{\prime}$, all having the eigenvalue $c_{0}^{2}$, are any complete (in $\vec{r}_{0}$ space) orthonormal set $\left\{x_{\mu}\right\}$. From the normalized function $\mathrm{w}_{1}\left(\overrightarrow{\mathrm{r}}_{1}\right)$, an orthonormal set $\left\{\mathrm{w}_{\nu}\left(\overrightarrow{\mathrm{r}}_{1}\right)\right\}$ can be built up that is complete for functions of $\overrightarrow{\mathbf{r}}_{1}$. Then the eigenstates of $\overline{\mathbf{K}}^{\prime}$ may be enumerated as the set $\mathrm{x}_{\mu} \mathrm{w}_{1}$ belonging to the eigenvalue $c_{0}^{2}$, plus the set $x_{\mu} w_{\nu}, \nu=2,3, \ldots$, belonging to the eigenvalue zero. Also required are the relations

$$
\mathrm{K}_{2}\left(\mathrm{x}_{\mu} \mathrm{w}_{\nu}\right)=\delta_{\nu 1}\left(\mathrm{c}_{\mathrm{o}} \mathrm{x}_{\mu}\right)
$$

and

$$
\mathrm{K}_{2}^{\dagger} \mathrm{x}_{\mu}=\mathrm{c}_{\mathrm{o}} \mathrm{x}_{\mu} \mathrm{w}_{1}
$$

Operator products involving $\Pi_{\alpha}$ and $\Pi_{c}$ may be written in the separable form as, for example,

$$
\begin{gathered}
\left.\Pi_{\alpha} \Pi_{\mathrm{c}}=\mathrm{K}_{2} \mid \phi\right)\left(\psi\left|=\mathrm{c}_{\mathrm{o}}{ }_{\mathrm{r}_{0}}\right| \phi\right)\left(\omega_{1} \psi \mid\right. \\
\left.\Pi_{\alpha} \Pi_{\mathrm{c}} \Pi_{\alpha}=\mathrm{K}^{\prime} \mid \phi\right)\left(\phi \mid=\mathrm{c}_{\mathrm{o}}^{2} \Pi_{\alpha}\right.
\end{gathered}
$$

and

$$
\left.\Pi_{\mathrm{c}} \Pi_{\alpha} \Pi_{\mathrm{c}}=\overline{\mathrm{K}}^{\prime} \mid \psi\right)(\psi \mid
$$

The required operational rules are simply

$$
\begin{gathered}
\Pi_{\alpha}(\rho \phi)=\rho \phi \\
\Pi_{\alpha}(\zeta \psi)=\left(\mathrm{K}_{2} \zeta\right) \phi \\
\Pi_{\mathrm{c}}(\rho \phi)=\left(\mathrm{K}_{2}^{\dagger} \rho\right) \psi \\
\Pi_{\mathrm{c}}(\zeta \psi)=\zeta \psi
\end{gathered}
$$


Now the projector onto the subspace $\varsigma_{\alpha+c}$ can be constructed from any of the equivalent forms

$$
\begin{gathered}
\Pi_{\alpha+c}=\Pi_{c}+\Pi_{\alpha p c} \\
\Pi_{\alpha+c}=\Pi_{\alpha}+\Pi_{c p \alpha} \\
\Pi_{\alpha+c}=R_{\alpha}^{\prime}+R_{c}
\end{gathered}
$$

where $R_{\alpha} \Psi=F^{\prime} \phi$ and $R_{c} \Psi=T \psi$. The form (eq. (70)) follows from the fact that $\varsigma_{\alpha+c}$ is the direct sum of $\Phi_{c}$ and the largest subspace of $\varsigma_{\alpha}$ that is orthogonal to $\mathscr{S}_{c^{*}}$ The subspace $\mathscr{S}_{\alpha}$ is spanned by the set $\left\{\mathbf{X}_{\mu} \phi\right\}$. The unit vectors $N\left(1-\Pi_{c}\right) x_{\mu} \phi$ $=\mathrm{NX}_{\mu}(\phi-\mathrm{g} \psi)$, each with the normalization constant $\mathrm{N}=\left(1-\mathrm{c}_{\mathrm{o}}^{2}\right)^{-1 / 2}$, are an orthonormal set that spans $5_{\alpha p c} \cdot$ Note that $c_{0}^{2} \leqq 1$. The equality sign holds only if $\phi$ is proportional to $\psi$, in which case, $\mathscr{S}_{\alpha+c}=\mathscr{5}_{\mathrm{c}}$. Hence, the required projector for equation (70) is

$$
\begin{aligned}
\Pi_{\alpha p c} & =N^{2} \Sigma_{\mu}\left(1-\Pi_{c}\right)\left|x_{\mu} \phi\right\rangle\left\langle x_{\mu} \phi\right|\left(1-\Pi_{c}\right) \\
& =N^{2}\left(1-\Pi_{c}\right) \Pi_{\alpha}\left(1-\Pi_{c}\right) \\
& \left.=1_{r_{0}} \mid \varphi_{0}{ }^{\prime}\right)\left(\varphi_{0}^{\prime} \mid\right.
\end{aligned}
$$

where $\varphi_{\mathrm{o}}{ }^{\prime}=\mathrm{N}(\phi-\mathrm{g} \psi)$. The orthonormal vectors $\mathrm{z}_{\mu \nu}=\mathrm{x}_{\mu} \mathrm{w}_{\nu} \psi$ span $\mathfrak{S}_{\mathrm{c}}$, and their normalized components orthogonal to $\$_{\alpha}$ are

$$
\begin{gathered}
\overline{\mathrm{z}}_{\mu 1}=\mathrm{N}\left(1-\Pi_{\alpha}\right) \mathrm{z}_{\mu 1}=\mathrm{NX}_{\mu}\left(\mathrm{w}_{1} \psi-\mathrm{c}_{\mathrm{o}} \phi\right) \\
\overline{\mathrm{z}}_{\mu \nu}=(1-\Pi) \mathrm{z}_{\mu \nu}=\mathrm{z}_{\mu \nu} \quad \nu=2,3, . .
\end{gathered}
$$

The vectors $\overline{\mathrm{z}}_{\mu \nu}$ are orthogonal to one another as well, so that 


$$
\begin{aligned}
\Pi_{c p \alpha} & =\sum_{\mu=1}^{\infty} \sum_{\nu=1}^{\infty}\left|\overline{\mathrm{z}}_{\mu \nu}\right\rangle\left\langle\overline{\mathrm{z}}_{\mu \nu}\right| \\
& =\sum_{\mu=1}^{\infty}\left|\overline{\mathrm{z}}_{\mu 1} \overline{\mathrm{z}}_{\mu 1}\right|-\left|\mathrm{z}_{\mu 1}\right\rangle\left\langle\mathrm{z}_{\mu 1} \mu+\sum_{\nu=1}^{\infty}\left|\mathrm{z}_{\mu \nu} \mathrm{z}_{\mu \nu}\right|\right. \\
& =1_{\mathbf{r}_{0}}\left[\mid \varphi_{\mathrm{o}}{ }^{\prime \prime}\right)\left(\varphi_{\mathrm{o}}{ }^{\prime \prime}|-| \mathrm{w}_{1} \psi\right)\left(\mathrm{w}_{1} \psi \mid\right]+\Pi_{\mathrm{c}}
\end{aligned}
$$

where $\varphi_{0}^{\prime \prime}=\mathrm{N}\left(w_{1} \psi-c_{0} \phi\right)$. The projector can also be written in the form

$$
\Pi_{c p \alpha}=\left(1-\Pi_{\alpha}\right) \bar{\Lambda}^{\prime}\left(1-\Pi_{\alpha}\right)
$$

in which

$$
\begin{aligned}
\bar{\Lambda}^{\prime} & =\sum_{\mu=1}^{\infty} \mathrm{N}^{2}\left|\mathrm{z}_{\mu 1}\right\rangle\left\langle\mathrm{z}_{\mu 1}\left|+\sum_{\nu=2}^{\infty}\right| \mathrm{z}_{\mu \nu}\right\rangle\left\langle\mathrm{z}_{\mu \nu}\right| \\
& \left.=\left[\left(\mathrm{N}^{2}-1\right) 1_{\mathrm{r}_{0}} \mid \mathrm{w}_{1}\right)\left(\mathrm{w}_{1}\left|+1_{\mathrm{r}_{0}} \sum_{\nu=1}^{\infty}\right| \mathrm{w}_{\nu}\right)\left(\mathrm{w}_{\nu} \mid\right] \mid \psi\right)(\psi \mid \\
& =\mathrm{N}^{2} \Pi_{\mathrm{c}} \Pi_{\alpha} \Pi_{\mathrm{c}}+\Pi_{\mathrm{c}}
\end{aligned}
$$

By the now familiar procedure, form (72) is obtained from equations (like eq. (31), but with $\Pi_{c}$ instead of $\Pi_{\beta}$ ) that impose the condition that $\mathscr{5}_{\alpha+c}$ contain the subspaces $\varsigma_{\alpha}$ and $\varsigma_{c}$ but no more. Accordingly,

$$
\left.\begin{array}{c}
\mathrm{R}_{\alpha}{ }^{\prime}=\Pi_{\alpha} \Lambda^{\prime} \Pi_{\alpha}\left(1-\Pi_{\mathrm{c}}\right) \\
\mathrm{R}_{\mathrm{c}}=\Pi_{\mathrm{c}} \bar{\Lambda}^{\prime} \Pi_{\mathrm{c}}\left(1-\Pi_{\alpha}\right)
\end{array}\right\}
$$

where $\Lambda^{\prime}$ and $\bar{\Lambda}^{\prime}$ satisfy the respective relations

$$
\Pi_{\alpha} \Lambda^{\prime} \Pi_{\alpha}\left(1-\Pi_{c}\right) \Pi_{\alpha}=\Pi_{\alpha}
$$




$$
\Pi_{c} \bar{\Lambda}^{\prime} \Pi_{c}\left(1-\Pi_{\alpha}\right) \Pi_{c}=\Pi_{c}
$$

Inserting the separable forms $\left.\Lambda^{\prime}=L^{\prime} \mid \phi\right)\left(\phi \mid\right.$ and $\left.\bar{\Lambda}^{\prime}=\bar{L}^{\prime} \mid \psi\right)(\psi \mid$ into equations (76) and (77), respectively, shows that

$$
L^{\prime}=\left(1_{r_{0}}-K^{\prime}\right)^{-1}
$$

and

$$
\overline{\mathrm{L}}^{\prime}=\left({ }_{\mathbf{r}_{0}}{ }^{\mathbf{r}_{1}}-\overline{\mathrm{K}}^{\prime}\right)^{-1}
$$

From the structure of $K^{\prime}$ and $\overline{\mathbf{K}}^{\prime}$ given by equation (68), it follows that

$$
L^{\prime}=N^{2} 1_{r_{0}}
$$

and

$$
\bar{L}^{\prime}=1_{\mathrm{r}_{0}}\left[\mathrm{~N}^{2} \mid \mathrm{w}_{1}\right)\left(\mathrm{w}_{1}\left|+\sum_{\nu=2}^{\infty}\right| \mathrm{w}_{\nu}\right)\left(\mathrm{w}_{\nu} \mid\right]
$$

The expression for $\bar{L}$ is its expansion in the eigenvectors of $\bar{K}^{\prime}$ and confirms the equivalence of the definitions (eqs. (74) and (77)) of $\bar{\Lambda}^{\prime}$. The condition $\Pi_{\alpha}\left(1-\Pi_{\alpha+c}\right)=0$ yields the identity $\Pi_{\alpha}=\mathrm{R}_{\alpha}{ }^{\prime}+\Pi_{\alpha} \mathrm{R}_{\mathrm{c}}$. Hence, equation (72) can be written as $\Pi_{\alpha+\mathrm{c}}=\Pi_{\alpha}+\left(1-\Pi_{\alpha}\right) \mathrm{R}_{\mathrm{c}}$, which, with equations (75) and (73), shows the equivalence to equation (71) explicitly. Note that the set $\mathrm{w}_{\nu}$ serves only as an intermediate construct for deriving the projection operators. All $\mathrm{w}_{\nu}$ for which $\nu \geq 2$ can be eliminated from the final forms with the help of the closure property.

\section{MULTICHANNEL PROJECTION OPERATORS}

\section{The Projector $\Pi_{A+B^{\prime}}$}

The two-channel projection operators given in the preceding section are easily generalized to any number of $\alpha$ and $\beta$ channels. In this section, the first case treated is the extension for the projector $\Pi_{\alpha+\beta^{*}}$. The corresponding discussion for the projector 
$\Pi_{A^{\prime}+B}$ closely parallels that for $\Pi_{\alpha+\beta^{\prime}}$ and need not be given explicitly. The redefined $\beta$ channel subspace $\mathfrak{S}_{B^{\prime}}$ is the direct sum of the orthogonal subspaces $\mathfrak{S}_{\beta^{\prime} \mathrm{n}}, \mathrm{n} \subset \mathrm{B}$, and has the projector

$$
\Pi_{B^{\prime}}=\sum_{n \subset B} \Pi_{\beta^{\prime} n}
$$

where $\Pi_{\beta^{\prime} n}$ is given by equation (43). Now the projection operator onto the space ${ }^{5} \mathrm{~A}+\mathrm{B}$, spanned by all vectors of $\mathfrak{S}_{\mathrm{A}}$ and $\mathfrak{S}_{\mathrm{B}}$, combined is required. The projector may be written as

$$
\begin{gathered}
\Pi_{\mathrm{A}+\mathrm{B}^{\prime}}=\Pi_{\mathrm{A}}+\Pi_{\mathrm{B}^{\prime} \mathrm{pA}} \\
\Pi_{\mathrm{A}+\mathrm{B}^{\prime}}=\Pi_{\mathrm{B}^{\prime}}+\Pi_{\mathrm{ApB^{ \prime }}} \\
\Pi_{\mathrm{A}+\mathrm{B}^{\prime}}=\widetilde{\mathrm{R}}_{\mathrm{A}^{\prime}}+\mathrm{R}_{\mathrm{B}^{\prime}}
\end{gathered}
$$

where the operators in equation $(80)$ have the properties

$$
\left.\begin{array}{r}
\widetilde{R}_{A^{\Psi}}=\sum_{\mathrm{m} \subset A} F_{m} \phi_{m} \\
\mathrm{R}_{\mathrm{B}^{\prime}} \Psi=\sum_{\mathrm{n} \subset \mathrm{B}} \mathrm{G}_{\mathrm{n}}(\chi \psi)_{\mathrm{n}}
\end{array}\right\}
$$

Sums over $m$ and $n$ with unspecified limits will be understood, henceforth, to range over the selected values contained in the sets $\mathrm{A}$ and $\mathrm{B}$ and to comprise $\mathrm{M}$ and $\mathrm{N}$ terms, respectively.

The transformation functions are given by equations (44) and (45) as

$$
\begin{aligned}
\mathrm{J}_{1}\left(\overrightarrow{\mathrm{r}}_{0}, \overrightarrow{\mathrm{r}}_{0^{\prime}} ; \mathrm{m}, \mathrm{n}\right) & =\left\langle\overrightarrow{\mathrm{r}}_{0}, \phi_{\mathrm{m}} \mid \overrightarrow{\mathrm{r}}_{0^{\prime}},(\chi \psi)_{\mathrm{n}}\right\rangle \\
& =\delta\left(\overrightarrow{\mathrm{r}}_{0}-\overrightarrow{\mathrm{r}}_{0}^{\prime}\right) \mathrm{h}_{\mathrm{mn}}\left(\overrightarrow{\mathrm{r}}_{0}\right)
\end{aligned}
$$

where 


$$
h_{m n}\left(\vec{r}_{0}\right)=\int g_{j m} *\left(\vec{r}_{1}\right) \chi_{i}\left(\vec{r}_{0}-\vec{r}_{1}^{\prime}\right) d \vec{r}_{1}^{\prime}
$$

The array of operators (in $\vec{r}_{0}$ space) $J_{1}(\mathrm{mn})$ can be represented as a matrix

$$
\mathrm{J}_{1}=\mathbf{1}_{\mathbf{r}_{0}} \mathrm{~h}
$$

where $h\left(\vec{r}_{0}\right)$ is the $M \times N$ dimensional matrix whose elements are the functions given by equation (83). The iterates

$$
\begin{aligned}
J\left(\overrightarrow{\mathbf{r}}_{0}, \overrightarrow{\mathrm{r}}_{0^{\prime}} ; \mathrm{m}, \mathrm{m}^{\prime}\right) & =\Sigma_{\mathrm{n}} \int \mathrm{J}_{1}\left(\overrightarrow{\mathrm{r}}_{0}, \overrightarrow{\mathrm{r}}_{0^{\prime}} ; \mathrm{m}, \mathrm{n}\right) \mathrm{d} \overrightarrow{\mathrm{r}}_{0^{\prime \prime}}{ }_{1}^{\dagger}\left(\overrightarrow{\mathrm{r}}_{0^{\prime \prime}}, \overrightarrow{\mathrm{r}}_{0^{\prime}} ; \mathrm{n}, \mathrm{m}^{\prime}\right) \\
& =\left\langle\overrightarrow{\mathrm{r}}_{0}, \phi_{\mathrm{m}}\left|\Pi_{\mathrm{B}^{\prime}}\right| \overrightarrow{\mathrm{r}}_{0^{\prime}}, \phi_{\mathrm{m}^{\prime}}\right\rangle
\end{aligned}
$$

represent an $M$-dimensional square array of operators (in $\vec{r}_{0}$ space) $\mathrm{J}\left(\mathrm{m}, \mathrm{m}^{\prime}\right.$ ), which can be written as $J=1_{\mathbf{r}_{0}} \mathbf{h}^{\dagger}$. Similarly, $\bar{J}=J_{1}^{\dagger} J_{1}$ gives an $\mathrm{N}$-dimensional square array $\bar{J}=1_{r_{0}} h^{\dagger} h$. Note that $J\left(m, m^{\prime}\right)^{\dagger}=J\left(m^{\prime}, m\right), J\left(n, n^{\prime}\right)^{\dagger}=\bar{J}\left(n^{\prime}, n\right)$. Only the diagonal operators of the arrays $J, \vec{J}$ are Hermitian. A normalized eigenvector of $J\left(m, m^{\prime}\right)$ and $\bar{J}\left(n, n^{\prime}\right)$ is $\delta_{a}$, with respective eigenvalues $\left[h(\vec{a}) h^{\dagger}(\vec{a})\right]_{m m^{\prime}}$ and $\left[h^{\dagger}(\vec{a}) h(\vec{a})\right]_{h n^{\prime}}$. In addition, $\delta_{\mathrm{a}}$ satisfies the eigenvalue equations

$$
\begin{aligned}
\mathrm{J}_{1}(\mathrm{~m}, \mathrm{n}) \delta_{\mathrm{a}} & =\mathrm{h}_{\mathrm{mn}}(\overrightarrow{\mathrm{a}}) \delta_{\mathrm{a}} \\
\mathrm{J}_{1}(\mathrm{~m}, \mathrm{n})^{\dagger} \delta_{\mathrm{a}} & =\mathrm{h}_{\mathrm{mn}}{ }^{*(\vec{a}) \delta_{\mathrm{a}}}
\end{aligned}
$$

Once more, the operator products are easily obtained in the separable form

$$
\begin{aligned}
& \left.\Pi_{A} \Pi_{B^{\prime}}=\Sigma_{m} \Sigma_{n} J_{1}(m, n) \mid \phi_{m}\right)\left([x \psi]_{n} \mid\right. \\
& \left.\Pi_{A} \Pi_{B^{\prime}}, \Pi_{A}=\Sigma_{m^{\prime}} \Sigma_{m^{\prime}} J\left(m, m^{\prime}\right) \mid \phi_{m}\right)\left(\phi_{m}, \mid\right. \\
& \left.\Pi_{B^{\prime}}, \Pi_{A} \Pi_{B^{\prime}}=\Sigma_{n^{\prime}} \Sigma_{n^{\prime}} \bar{J}\left(n, n^{\prime}\right) \mid[\chi \psi]_{n}\right)\left([\chi \psi]_{n^{\prime}} \mid\right.
\end{aligned}
$$

The required operator rules also follow directly 


$$
\left.\begin{array}{c}
\Pi_{\alpha m^{\prime}}\left(\rho \phi_{\mathrm{m}}\right)=\delta_{\mathrm{mm}^{\prime}} \rho \phi_{\mathrm{m}} \\
\Pi_{\alpha \mathrm{m}}\left[\rho(\chi \psi)_{\mathrm{n}}\right]=\left[\mathrm{J}_{1}(\mathrm{mn}) \rho\right] \phi_{\mathrm{m}} \\
\Pi_{\beta^{\prime} \mathrm{n}^{\prime}}\left(\rho \phi_{\mathrm{m}}\right)=\left[\mathrm{J}_{1}(\mathrm{mn})^{\dagger} \rho\right](\chi \psi)_{\mathrm{n}} \\
\Pi_{\beta^{\prime} \mathrm{n}^{,}}\left[\rho(\chi \psi)_{\mathrm{n}}\right]=\delta_{\mathrm{n}^{\prime} \mathrm{n}}(\chi \psi)_{\mathrm{n}}
\end{array}\right\}
$$

The subspace $S_{\beta^{\prime} n}$ is spanned by the orthonromal set $\left\{y_{a n} \equiv \delta_{a}(\chi \psi)_{n}\right\}$. The $N$ sets, each having a value of $\mathrm{n} \subset \mathrm{B}$, are orthogonal to one another and provide an orthonormal basis for $\mathfrak{S}_{\mathrm{B}}$. . The basis vectors $\mathrm{y}_{\text {an }}$ have components orthogonal to $\mathfrak{S}_{\mathrm{A}}$ given by

$$
\begin{aligned}
\bar{y}_{a n} & =\left(1-\Pi_{A}\right) y_{a n}=y_{a n}-\Sigma_{m} h_{m n}(\vec{a}) x_{a m} \\
& =\delta_{a} \omega_{n}
\end{aligned}
$$

in which $\mathrm{x}_{\mathrm{am}} \equiv \delta_{\mathrm{a}} \phi_{\mathrm{m}}, \omega_{\mathrm{n}} \equiv(\chi \psi)_{\mathrm{n}}-\Sigma_{\mathrm{m}} \mathrm{h}_{\mathrm{mn}} \phi_{\mathrm{m}}$, and $\mathrm{h}_{\mathrm{mn}}$ is understood to be a vector in $\overrightarrow{\mathbf{r}}_{0}$ space. The operator rules (eq. (87)) and equations (85) and (86) have been used. The scalar product

$$
\begin{aligned}
\left\langle\bar{y}_{a n} \mid \bar{y}_{a^{\prime} n^{\prime}}\right\rangle & =\left\langle y_{a n} \mid y_{a^{\prime} n^{\prime}}\right\rangle-\left\langle\Pi_{A^{a n}} y_{a} \mid \Pi_{A^{\prime}} y_{a^{\prime} n^{\prime}}\right\rangle \\
& =\delta_{n n^{\prime}} \delta\left(\vec{a}-\vec{a}^{\prime}\right)-\Sigma_{m^{\prime}} h_{m n} *(\vec{a}) h_{m n^{\prime}}(\vec{a}) \delta\left(\vec{a}-\vec{a}^{\prime}\right) \\
& =\left[1_{N}-h^{\dagger}(\vec{a}) h(\vec{a})\right]_{n n^{\prime}} \delta\left(\vec{a}-\vec{a}^{\prime}\right)
\end{aligned}
$$

shows that the sets $\left\{\overline{\mathbf{y}}_{\mathrm{an}}\right\}$ are not orthogonal.

To each value of $\vec{a}$ there corresponds a set of $N$ vectors $\bar{y}_{a n}, n \subset B$. If the vectors of this original set are independent, any orthonormal set constructed from them will also contain $\mathrm{N}$ vectors. The scalar products given by a set of $\mathrm{N}$ vectors form an $\mathrm{N}$-dimensional Hermitian matrix. An orthonormal set of vectors can be obtained from the original vectors by the unitary transformation matrix $U(\vec{a})$ that diagonalizes the matrix. If the Hermitian matrix is interpreted to be the representation in some (orthonormal) basis of an Hermitian operator, then in the well-known way $U(\vec{a})$ transforms to the new basis made up of normalized eigenvectors of the operator. (The columns of $U$ are the components of these eigenvectors in the old basis.) The same transformation on the original set of $\mathrm{N}$ vectors yields an orthonormal set. Such orthonormal sets, 
obtained for each value of $\vec{a}$, span the subspace $\mathfrak{S}_{\mathrm{B}^{\prime} \mathrm{pA}}$ and provide a convenient expression for its projector.

To obtain an orthonormal basis for the subspace $S_{\mathrm{B}^{\prime} \mathrm{pA}}$, consider the diagonalization of the matrix $1_{N}-h^{\dagger}(\vec{a}) h(\vec{a})$. For the time being, the dependence on $\vec{a}$ of the matrices and eigenvalues that arise in the discussion will be suppressed. The matrix $h^{\dagger} h$ is Hermitian, so that there is a unitary matrix $U$ that diagonalizes it. Thus, write $\mathrm{U}^{\dagger} h^{\dagger} h U=h_{D}=\left(\delta_{n n}, h_{n}\right)$, where $h_{D}$ is a diagonal, real (N-dimensional) matrix. Because $\mathrm{h}^{\dagger} \mathrm{h}$ is nonnegative, the eigenvalues $h_{n}$ are also nonnegative. The columns of $U$ are normalized eigenvectors of $h^{\dagger} h$. A certain lack of uniqueness to the matrix $U$ exists. The matrix $\mathrm{UU}_{\mathrm{D}}$, where $\mathrm{U}_{\mathrm{D}}$ is any diagonal, unitary matrix, will serve as well and corresponds merely to multiplication of the column eigenvectors of $U$ by phase factors. The Hermitian matrices are defined as $d=1_{M}-h^{\dagger}$ and $d=1_{N}-h^{\dagger} h$ and are related as follows:

$$
\left.\begin{array}{c}
\mathrm{dh}=\mathrm{h} \overline{\mathrm{d}} \\
h \bar{d}^{-1}=\mathrm{d}^{-1} \mathrm{~h} \\
\mathrm{~d}^{-1}=1_{M}+h \bar{d}^{-1} \mathrm{~h}^{\dagger}
\end{array}\right\}
$$

The reciprocal of the relation $U^{\dagger} \bar{d} U=1_{N}-h_{D}$ gives

$$
\overline{\mathrm{d}}^{-1}=\mathrm{U}\left(1_{\mathrm{N}}-\mathrm{h}_{\mathrm{D}}\right)^{-1} \mathrm{U}^{\dagger}
$$

The linear combinations of the vectors (eq. (88))

$$
\mathrm{Y}_{\mathrm{an}}=\mathrm{N}_{\mathrm{n}}(\mathrm{a}) \Sigma_{\mathrm{n}^{\prime}}, \mathrm{U}_{\mathrm{n}^{\prime} \mathrm{n}}(\mathrm{a}) \overline{\mathrm{y}}_{\mathrm{an}},
$$

satisfy the orthogonality relation

$$
\left\langle\mathrm{Y}_{\mathrm{an}} \mid \mathrm{Y}_{\mathrm{a}^{\prime} \mathrm{n}^{\prime}}\right\rangle=\left[\mathrm{N}_{\mathrm{n}}(\overrightarrow{\mathrm{a}})\right]^{2}\left[1-\mathrm{h}_{\mathrm{n}}(\overrightarrow{\mathrm{a}})\right] \delta_{\mathrm{nn}}, \delta\left(\overrightarrow{\mathrm{a}}-\overrightarrow{\mathrm{a}}^{\prime}\right)
$$

Because a representative of $\delta_{a}$ is the delta function $\delta\left(\vec{r}_{0}-\vec{a}\right)$, the vector $Y_{a n}$ can be written $\mathrm{Y}_{\text {an }}=\delta_{\mathrm{a}}\left(\Omega_{\mathrm{n}}\right)$, where

$$
\Omega_{n}=N_{n} \Sigma_{n}, U_{n^{\prime} n}\left[(\chi \psi)_{n^{\prime}}-\Sigma_{m} h_{m n}, \phi_{m}\right]
$$


and the quantities $N_{n} U_{n^{\prime} n}$ and $N_{n} U_{n} n^{h} n_{n}$, become functions of $\vec{r}_{0}$ in the coordinate representation of $Y_{a n}$. The normalization $N_{n}(\vec{a})=\left[1-h_{n}(\vec{a})\right]^{-1 / 2}$ makes the $Y_{\text {an }}$ orthonormal, so that

$$
\begin{gathered}
\Pi_{B^{\prime} p A}=\int d \vec{a} \Sigma_{n}\left|Y_{a n}\right\rangle\left\langle Y_{a n}\right| \\
\left.\Pi_{B^{\prime} p A}=1_{r_{0}} \Sigma_{n} \mid \Omega_{n}\right)\left(\Omega_{n} \mid\right.
\end{gathered}
$$

If $h_{n}(\vec{a})$ ever assumed the value 1 , the corresponding vector $Y_{\text {an }}$ would be a null vector, which implies that the vectors $\overline{\mathrm{y}}_{\text {an }}$ and $\mathrm{n} \subset \mathrm{B}$, are not independent. Exclude this possibility, and assume that the matrix $\bar{d}(\vec{a})$ is never singular for any value of $\vec{a}$. Then, equation (93) can be expressed, with the help of equation (90), as

$$
\begin{aligned}
& \left.\Pi_{B^{\prime} p A}=\int d \vec{a} \Sigma_{n} \Sigma_{n^{\prime}}\left|\bar{y}_{a n^{\prime}}\right\rangle \bar{d}^{-1}(\vec{a})\right]_{n n^{\prime}}\left\langle\bar{y}_{a n^{\prime}}\right| \\
& \left.=1_{\mathbf{r}_{0}} \Sigma_{n \Sigma_{n^{\prime}}} \mid \omega_{n}\right)\left(\bar{d}^{-1}\right)_{n n^{\prime}}\left(\omega_{n^{\prime}} \mid\right. \\
& =1_{r_{0}}\left[\Sigma_{n} \Sigma_{n^{\prime}} \mid[\chi \psi]_{n^{\prime}}\right)\left(\bar{d}^{-1}\right)_{n n^{\prime}}\left(\omega_{n^{\prime}}\left|-\Sigma_{m^{n}} \Sigma_{n}\right| \phi_{m}\right)\left(h \bar{d}^{-1}\right)_{m n}(\chi \chi \psi]_{n} \mid
\end{aligned}
$$

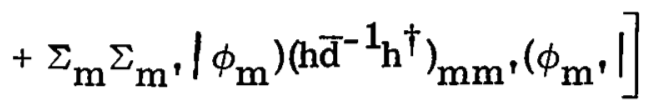

The matrix $U$ has dropped out of the form (eq. (95)), which shows that the arbitrariness in the choice of $U$ is not significant.

The form (eq. (80)) can be derived from the relations analogous to equation (59), with $\alpha$ and $\beta^{\prime}$ replaced by $A$ and $B^{\prime}$, respectively. The derivation leads to expressions for $\widetilde{R}_{A}$ and $R_{B}$, that are the direct analogs of equations (60), where $\Lambda_{O}$ and $\bar{\Lambda}_{O}$ are now operators in $\mathfrak{5}_{A}$ and $\mathfrak{s}_{\mathrm{B}}$, respectively, and can be written as

$$
\left.\Lambda_{\mathrm{O}}=\Sigma_{\mathrm{m}} \Sigma_{\mathrm{m}}, \mathrm{O}_{\mathrm{mm}}, \mid \phi_{\mathrm{m}}\right)\left(\phi_{\mathrm{m}}, \mid\right.
$$

and

$$
\left.\bar{\Lambda}_{\mathrm{O}}=\Sigma_{\mathrm{n}} \Sigma_{\mathrm{n}}, \overline{\mathrm{O}}_{\mathrm{nn}}, \mid[\chi \psi]_{\mathrm{n}}\right)\left([\chi \psi]_{\mathrm{n}}, \mid\right.
$$

Both $O_{m m}$, and $\bar{O}_{n n}$, are Hermitian operators in $\vec{r}_{0}$ space, with matrix elements $\mathrm{O}_{\mathrm{mm}}\left(\overrightarrow{\mathrm{r}}_{0^{\prime}}, \overrightarrow{\mathrm{r}}_{0^{\prime}}\right)=\left\langle\overrightarrow{\mathrm{r}}_{0}, \phi_{\mathrm{m}}\left|\Lambda_{\mathrm{M}}\right| \overrightarrow{\mathrm{r}}_{0^{\prime}}, \phi_{\mathrm{m}^{\prime}}\right\rangle$, and so forth. When the expressions for $\Lambda_{\mathrm{O}}$ 
and $\bar{\Lambda}_{\mathrm{O}}$ are inserted in their defining equations, which are the direct analogs of equation (61), the equations for the operator arrays $O$ and $\bar{O}$ are obtained. In matrix notation, these take the form

$$
O\left(1_{r_{0}}{ }^{1}-J\right)=1_{r_{0}}{ }^{1} \mathbf{M}
$$

and

$$
\overline{\mathrm{O}}\left(\mathbf{1}_{\mathbf{r}_{0}}{ }^{1_{N}}-\overline{\mathrm{J}}\right)={ }_{\mathbf{r}_{0}}{ }^{1_{N}}
$$

from which it is clear that

$$
\mathrm{O}=\mathrm{d}^{-1} \mathbf{1}_{\mathbf{r}}
$$

and

$$
\overline{\mathrm{O}}=\overline{\mathrm{d}}^{-1} \mathbf{1}_{\mathbf{r}}
$$

Collect the results to obtain

$$
\begin{aligned}
& \left.\widetilde{\mathrm{R}}_{\mathrm{A}}=1_{\mathbf{r}_{0}} \Sigma_{\mathrm{m}} \Sigma_{\mathrm{m}^{\prime}}\left(\mathrm{d}^{-1}\right)_{\mathrm{mm}} \cdot \mid \phi_{\mathrm{m}}\right)\left(\phi_{\mathrm{m}} \cdot \mid\left(1-\Pi_{\mathrm{B}^{\prime}}\right)\right. \\
& =1_{\mathbf{r}_{0}}\left\{\Sigma_{\mathrm{m}} \Sigma_{\mathrm{m}^{*}}\left(\mathrm{~d}^{-1}\right)_{\mathrm{mm}} \cdot \mid \phi_{\mathrm{m}}\right)\left(\phi_{\mathrm{m}} \cdot \mid-\Sigma_{\mathrm{m}} \Sigma_{\mathrm{n}}\left(\mathrm{d}^{\left.-1_{\mathrm{h}}\right)_{\mathrm{mn}}} \mid \phi_{\mathrm{m}}\right)\left([\chi \psi]_{\mathrm{n}} \mid\right\}\right.
\end{aligned}
$$

and

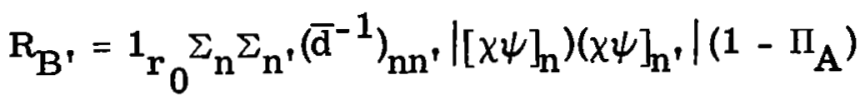

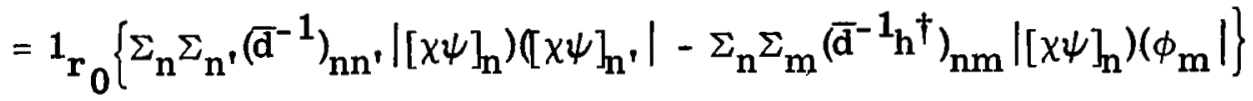

Thus, $\widetilde{R}_{A}$ and $R_{B^{\prime}}$, as seen immediately from their defining equations (the analogs of eqs. (60) and (61)), are idempotents and their product in either order vanishes. Comparison of equations (96) and (97) and equation (95), with the help of equation (89), verifies the equivalence of the forms (78) and (80). 


\section{The Projector of Chen and Mittleman}

The projection operator given by Chen and Mittleman is closely related to $\Pi_{A+B}$. In terms of the formalism presented in this report, Chen and Mittleman employ the channel subspace $\varsigma_{\bar{\beta}}$, defined as the space spanned by all vectors of the form $\rho(\eta \psi)_{n}$, where $\rho$ is a vector in $\vec{r}_{0}$ space and $(\eta \psi)_{n}=\eta_{n} \psi_{j}$. The component $\eta_{n}$ is defined by the representation

$$
\begin{gathered}
\left(\overrightarrow{\mathrm{r}}_{0^{\prime}}, \overrightarrow{\mathrm{r}}^{\prime} \mid \overrightarrow{\mathrm{r}}_{0}, \eta_{\mathrm{n}}\right)=\delta\left(\overrightarrow{\mathrm{r}}_{0^{\prime}}-\overrightarrow{\mathrm{r}}_{0}\right) \eta_{\mathrm{n}}\left(\hat{\mathrm{r}}_{0}, \overrightarrow{\mathrm{r}}^{\prime}\right) \\
\eta_{\mathrm{n}}\left(\hat{\mathrm{r}}_{0}, \overrightarrow{\mathrm{r}}\right) \equiv \exp \left(-\mathrm{i} \kappa_{\mathrm{n}} \hat{\mathrm{r}}_{0} \cdot \overrightarrow{\mathrm{r}} / 2\right) \chi_{\mathrm{i}}(\overrightarrow{\mathrm{r}})
\end{gathered}
$$

The projection operator for $\$ \bar{\beta}$ is

$$
\left.\Pi \bar{\beta} \mathbf{n}=1_{\mathbf{r}_{0}} \mid[\eta \psi]_{\mathbf{n}}\right)\left([\eta \psi]_{\mathbf{n}} \mid\right.
$$

The basis vectors for the subspace are represented by

$$
\left\langle\overrightarrow{\mathbf{r}}_{0^{\prime}}, \overrightarrow{\mathbf{r}}^{\prime}, \vec{\xi}^{\prime} \mid \overrightarrow{\mathbf{r}}_{0},(\eta \psi)_{\mathrm{n}}\right\rangle=\delta\left(\overrightarrow{\mathbf{r}}_{0^{\prime}}-\overrightarrow{\mathbf{r}}_{0}\right) \eta_{\mathrm{n}}\left(\hat{\mathbf{r}}_{0}, \overrightarrow{\mathbf{r}}^{\prime}\right) \psi_{\mathbf{j}}\left(\vec{\xi}^{\prime}\right)
$$

The subspaces ${ }^{5} \bar{\beta} \mathrm{n}$ are not mutually orthogonal. The scalar product of the basis vectors is

$$
\begin{aligned}
\left\langle\overrightarrow{\mathbf{r}}_{0},(\eta \psi)_{\mathbf{n}} \mid \overrightarrow{\mathbf{r}}_{0^{\prime}},(\eta \psi)_{\mathbf{n}^{\prime}}\right\rangle & =\delta_{\mathrm{jj}^{\prime}}\left(\overrightarrow{\mathbf{r}}_{0}, \eta_{\mathbf{n}} \mid \overrightarrow{\mathbf{r}}_{0^{\prime}}, \eta_{\mathbf{n}^{\prime}}\right) \\
& =\delta\left(\overrightarrow{\mathbf{r}}_{0}-\overrightarrow{\mathbf{r}}_{0^{\prime}}\right) \nu_{\mathrm{nn}^{\prime}}\left(\hat{\mathbf{r}}_{0}\right)
\end{aligned}
$$

where the functions defined by

$$
\nu_{n n^{\prime}}\left(\hat{\mathrm{r}}_{0}\right)=\delta_{\mathrm{jj}}, \int \exp \left[\frac{\hat{\mathrm{i}} \hat{\mathrm{r}}_{0} \cdot \overrightarrow{\mathrm{r}}^{\prime}\left(\kappa_{\mathrm{n}}-\kappa_{\mathrm{n}^{\prime}}\right)}{2}\right] \mathrm{d} \overrightarrow{\mathrm{r}}^{\prime} \chi_{\mathrm{i}^{*}}{ }^{*}\left(\overrightarrow{\mathrm{r}^{\prime}}\right) \chi_{\mathrm{i}^{\prime},},\left(\overrightarrow{\mathrm{r}^{\prime}}\right)
$$

form an N-dimensional matrix $\nu\left(\hat{\mathrm{r}}_{0}\right)$, which is Hermitian and has diagonal elements equal to 1 . Suppose the matrix $\nu\left(\hat{\mathrm{r}}_{0}\right)$ is diagonalized by the unitary matrix $\theta\left(\hat{\mathbf{r}}_{0}\right)$, that is, $\theta^{\dagger} \nu \theta=\left(\delta_{n n}, \nu_{n}\right) \equiv \nu_{D}$. Then, the linear combinations 


$$
\overline{\mathrm{Y}}_{\mathbf{r}_{0} \mathrm{n}}=\overline{\mathrm{N}}_{\mathbf{n}}\left(\overrightarrow{\mathrm{r}}_{0}\right) \Sigma_{\mathbf{n}^{\prime}} \theta_{\mathrm{n}^{\prime} \mathbf{n}}\left(\hat{\mathrm{r}}_{0}\right)\left|\overrightarrow{\mathrm{r}}_{0}^{\prime},(\eta \psi)_{\mathrm{n}^{\prime}}\right\rangle
$$

are orthogonal. Their scalar products are

$$
\left\langle\overline{\mathrm{Y}}_{\mathbf{r}_{0^{\mathrm{n}}}} \mid \overline{\mathrm{Y}}_{\mathbf{r}_{0^{\prime} \mathrm{n}^{\prime}}}\right\rangle=\overline{\mathrm{N}}_{\mathrm{n}}^{2}\left(\overrightarrow{\mathrm{r}}_{0}\right) \delta\left(\overrightarrow{\mathrm{r}}_{0}-\overrightarrow{\mathrm{r}}_{0^{\prime}}\right) \delta_{\mathrm{nn}}, \nu_{\mathrm{n}}\left(\hat{\mathrm{r}}_{0}\right)
$$

so that the normalization $\overline{\mathbf{N}}_{\mathrm{n}}\left(\overrightarrow{\mathrm{r}}_{0}\right)=\left[\nu_{\mathrm{n}}\left(\hat{\mathrm{r}}_{0}\right)\right]^{-1 / 2}$ makes them orthonormal. The matrix $\nu\left(\hat{\mathrm{r}}_{0}\right)$ is assumed to be nonsingular. The subspace $\mathscr{S}_{\overline{\mathrm{B}}}$, spanned by all subspaces $\mathscr{S}_{\bar{\beta} \mathrm{n}}$, $\mathrm{n} \subset \mathrm{B}$, has the projector operator

$$
\begin{aligned}
& \Pi_{\bar{B}}=\int d \vec{r}_{0} \Sigma_{n}\left|\bar{Y}_{r_{0} n^{n}}\right\rangle\left\langle\bar{Y}_{r_{0} n}\right| \\
& =\int \mathrm{d} \overrightarrow{\mathbf{r}}_{0} \Sigma_{\mathrm{n}} \Sigma_{\mathrm{n}^{\prime}}\left[\nu^{-1}\left(\hat{\mathrm{r}}_{0}\right)\right]_{\mathrm{n} n^{\prime}}\left|\overrightarrow{\mathrm{r}}_{0},(\eta \psi)_{\mathbf{n}}\right\rangle\left\langle\overrightarrow{\mathrm{r}}_{0},(\eta \psi)_{\mathrm{n}^{\prime}}\right|
\end{aligned}
$$

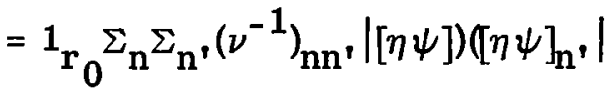

The relation $\nu^{-1}=\theta\left(\nu_{\mathrm{D}}\right)^{-1} \theta^{\dagger}$ was used in the first step.

The subspace $\sqrt{5}_{\mathrm{A}}+\overline{\mathrm{B}}$, spanned by $\sqrt{\mathrm{A}}_{\mathrm{A}}$ and $\mathfrak{S}_{\overline{\mathrm{B}}}$, combined, has the projection operator

$$
\begin{gathered}
\Pi_{A+\bar{B}}=\Pi_{A}+\Pi_{\overline{B p A}} \\
\Pi_{A+\bar{B}}=\Pi_{\bar{B}}+\Pi_{A p \bar{B}} \\
\Pi_{A+\bar{B}}=\bar{R}_{A}+R_{\bar{B}}
\end{gathered}
$$

where

$$
\begin{aligned}
& \overline{\mathrm{R}}_{\mathrm{A}} \Psi=\Sigma_{\mathrm{m}} \overline{\mathrm{F}}_{\mathrm{m}} \phi \mathrm{m} \\
& \overline{\mathrm{R}}_{\overline{\mathrm{B}}} \Psi=\Sigma_{\mathrm{n}} \overline{\mathrm{G}}_{\mathrm{n}}(\eta \psi)_{\mathrm{n}}
\end{aligned}
$$

and $\bar{F}_{m}, \bar{G}_{n}$ are vectors in $\vec{r}_{0}$ space. The channel transformation functions are 


$$
\left\langle\vec{r}_{0}, \phi_{\mathrm{m}} \mid \vec{r}_{0},(\eta \psi)_{\mathrm{n}}\right\rangle=\delta\left(\overrightarrow{\mathbf{r}}_{0}-\overrightarrow{\mathbf{r}}_{0}{ }^{\prime}\right) \Delta_{\mathrm{mn}}\left(\overrightarrow{\mathrm{r}}_{0}\right)
$$

with

$$
\Delta_{m n}\left(\vec{r}_{0}\right)=\int g_{j m} *\left(\vec{r}_{1}^{\prime}\right) \eta_{n}\left(\hat{r}_{0}, \vec{r}_{0}-\vec{r}_{1}\right) d \vec{r}_{1}^{\prime}
$$

Rather than construct $\Pi_{A+\bar{B}}$ by the orthogonalization process indicated in equation (101), only the expression (eq. (102)), which is the result of Chen and Mittleman, will be given for comparison with the corresponding form of $\Pi_{A+B}$.

The conditions

$$
\Pi_{A}\left(1-\Pi_{\bar{B}}\right)\left(1-\Pi_{A+\bar{B}}\right)=0
$$

and

$$
\Pi_{\bar{B}}\left(1-\Pi_{A}\right)\left(1-\Pi_{A+\bar{B}}\right)=0
$$

on the projector $\Pi_{\mathrm{A}+\overline{\mathrm{B}}}$, and the operator rules

$$
\Pi_{\mathrm{A}} \rho \varphi_{\mathrm{m}}=\rho \varphi_{\mathrm{m}}
$$

and

$$
\Pi_{\bar{B}} \rho(m \psi)_{n}=\rho(m \psi)_{n}
$$

lead directly to the expressions

$$
\bar{R}_{A}=\Pi_{A} \Lambda_{C} \Pi_{A}\left(1-\Pi_{\bar{B}}\right)
$$

and

$$
R_{\bar{B}}=\Pi_{\bar{B}} \bar{\Lambda}_{C} \Pi_{\bar{B}}\left(1-\Pi_{A}\right)
$$

Here, $\Lambda_{\mathbf{C}}$ and $\bar{\Lambda}_{\mathrm{C}}$ are Hermitian operators in ${ }_{5_{A}}$ and $\mathfrak{S}_{\overline{\mathrm{B}}}$, respectively, and satisfy the relations 


$$
\left.\begin{array}{l}
\Pi_{A} \Lambda_{C} \Pi_{A}\left(1-\Pi_{\bar{B}}\right) \Pi_{A}=\Pi_{A} \\
\Pi_{\bar{B}} \Lambda_{C} \Pi_{\bar{B}}\left(1-\Pi_{A}\right) \Pi_{\bar{B}}=\Pi_{\bar{B}}
\end{array}\right\}
$$

The following expressions can be written:

$$
\left.\begin{array}{l}
\left.\Lambda_{\mathrm{C}}=\Sigma_{\mathrm{m}} \Sigma_{\mathrm{m}}, \mathrm{C}_{\mathrm{mm}}, \mid \phi_{\mathrm{m}}\right)\left(\phi_{\mathrm{m}^{\prime}} \mid\right. \\
\left.\bar{\Lambda}_{\mathrm{C}}=\Sigma_{\mathrm{n}} \Sigma_{\mathrm{n}}, \overline{\mathrm{C}}_{\mathrm{nn}}, \mid[m \psi]_{\mathrm{n}}\right)(\eta \eta \psi]_{\mathrm{n}^{\prime}} \mid
\end{array}\right\}
$$

The operator products that will be required are

$$
\begin{aligned}
& \left.\Pi_{\mathrm{A}} \Pi_{\overline{\mathrm{B}}}=1_{\mathrm{r}_{0}} \Sigma_{\mathrm{m}} \Sigma_{\mathrm{n}} \mid \phi_{\mathrm{m}}\right)\left(\Delta \nu^{-1}\right)_{\mathrm{mn}}(\eta \eta \psi]_{\mathrm{n}} \mid \\
& \left.\Pi_{\mathrm{A}} \Pi_{\bar{B}} \Pi_{\mathrm{A}}=1_{\mathrm{r}_{0}} \Sigma_{\mathrm{m}} \Sigma_{\mathrm{m}} \cdot \mid \phi_{\mathrm{m}}\right)\left(\Delta \nu^{-1} \Delta^{\dagger}\right)_{\mathrm{mm}}\left(\phi_{\mathrm{m}} \mid\right.
\end{aligned}
$$

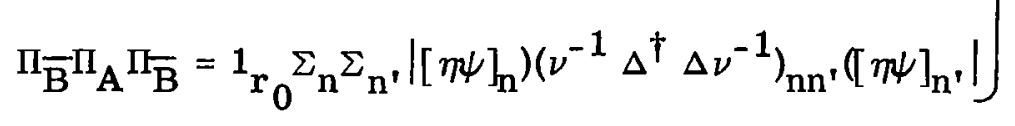

When equations (108) and (109) are inserted into the defining equations (eqs. (30)), the latter reduce directly to the equations

$$
\begin{aligned}
& \mathrm{C}\left(1_{\mathbf{r}_{0}}{ }_{1} \mathrm{M}-{ }_{\mathbf{r}_{0}} \Delta \nu^{-1} \Delta^{\dagger}\right)=1_{\mathbf{r}_{0}}{ }^{1} \mathrm{M} \\
& \overline{\mathbf{C}}\left(1_{\mathbf{r}_{0}}{ }^{1}{ }_{N}-1_{\mathbf{r}_{0}} \Delta^{\dagger} \Delta \nu^{-1}\right)=1_{\mathbf{r}_{0}} \nu^{-1}
\end{aligned}
$$

The solutions are

$$
\left.\begin{array}{c}
C=1_{r_{0}} B^{-1} \\
C=1_{r_{0}}(\nu \bar{B})^{-1}
\end{array}\right\}
$$

where the matrices $B\left(\vec{r}_{0}\right)$ and $\bar{B}\left(\vec{r}_{0}\right)$ are defined by the equations 


$$
\left.\begin{array}{l}
B=1_{M}-\Delta \nu^{-1} \Delta^{\dagger} \\
\bar{B}=1_{N}-\nu^{-1} \Delta^{\dagger} \Delta
\end{array}\right\}
$$

and satisfy the realtions

$$
\left.\begin{array}{c}
\mathrm{B} \Delta=\Delta \overline{\mathrm{B}} \\
\Delta \overline{\mathrm{B}}^{-1}=\mathrm{B}^{-1} \Delta \\
\mathrm{B}^{-1}=1_{M}+\Delta \overline{\mathrm{B}}^{-1} \nu^{-1} \Delta^{\dagger}
\end{array}\right\}
$$

When the solutions (eq. (110)) along with the first of equations (109) and its adjoint are employed in equations (105) and (106), the following equations are obtained:

$$
\begin{gathered}
\left.\overline{\mathrm{R}}_{\mathrm{A}}=1_{\mathrm{r}_{0}} \Sigma \mid \phi_{\mathrm{m}}\right)\left(\mathrm{B}^{-1}\right)_{\mathrm{mm}^{\prime}}\left(\phi_{\mathrm{m}^{\prime}}\left|-1_{\mathrm{r}_{0}} \Sigma\right| \phi_{\mathrm{m}}\right)\left(\mathrm{B}^{-1} \Delta \nu^{-1}\right)_{\mathrm{mn}}\left([\eta \psi]_{\mathrm{n}} \mid\right. \\
\left.\overline{\mathrm{R}}_{\mathrm{B}}=1_{\mathrm{r}_{0}} \Sigma \mid[\eta \psi]_{\mathrm{n}}\right)\left(\overline{\mathrm{B}}^{-1} \nu^{-1}\right)_{\mathrm{nn}^{\prime}}\left([\eta \psi]_{\mathrm{n}^{\prime}}\left|-1_{\mathrm{r}_{0}} \Sigma\right|[\eta \psi]_{\mathrm{n}}\right)\left(\overline{\mathrm{B}}^{-1} \nu^{-1} \Delta^{\dagger}\right)_{\mathrm{nm}}\left(\phi_{\mathrm{m}} \mid\right.
\end{gathered}
$$

The relations (eq. (112)) and the matrix $\mathrm{D} \equiv \nu \overline{\mathrm{B}}$, cast equations (113) and (114) into the form given by Chen and Mittleman.

The projector $\Pi_{A+\bar{B}}$ is not as convenient to apply as $\Pi_{A+B^{*}}$. Because of the exponential functions present, the overlap integrals (eq. (104)) are more complicated than the integrals (eq. (83)). For the same reason, the operators (eqs. (113) and (114)) are more difficult to employ than equations (96) and (97). Moreover, the components of $R_{\bar{B}} \Psi$ in equation (103) are not orthogonal, and the resulting coupled equations for the functions $\overline{\mathrm{F}}_{\mathrm{m}}\left(\overrightarrow{\mathrm{r}}_{0}\right)$ and $\overline{\mathrm{G}}_{\mathrm{n}}\left(\overrightarrow{\mathrm{r}}_{0}\right)$ cannot be manipulated into as convenient forms for numerical calculations as the equations for $\widetilde{F}_{m}$ and $G_{n}$. The boundary conditions on the functions $\bar{G}_{n}\left(\vec{r}_{0}\right)$ are somewhat more simple than those for the $G_{n}\left(\vec{r}_{0}\right)$, as shown in the section Asymptotic Properties. This last feature makes little difference in a numerical treatment, however. More complicated projectors, similar to $\Pi_{\mathrm{A}+\overline{\mathrm{B}}}$, are required only when the recoil of the target must be taken into account (ref. 6). 


\section{The Projector $\Pi_{\mathrm{A}+\mathrm{C}}$}

Recall that the subspace $\mathscr{S}_{\mathrm{cj}}$ is spanned by all vectors of the form $\zeta \psi_{j}$ (where each $\zeta$ is represented by a function of the variables $\vec{R}, \vec{r}$ or of $\vec{r}_{0}, \vec{r}_{1}$ ) and that it has the projection operator given in equation (66). Any selected set of states of the nucleus st-1 corresponds to a set $\mathrm{C}$ of $\mathrm{j}$ values. The subspace ${ }^{5_{\mathrm{C}}} \mathrm{C}$ is defined to be the direct sum of the orthogonal subspaces $\Im_{\mathrm{cj}}$ for all $\mathrm{j} \subset \mathrm{C}$ and has the projector

$$
\Pi_{\mathbf{C}}=\sum_{\mathbf{j} \subset \mathbf{C}} \Pi_{\mathbf{c j}}
$$

The generalization of the projector $\Pi_{\alpha+c}$ is the projection operator $\Pi_{A+C}$ for the subspace $\sqrt{5}_{\mathrm{A}+\mathrm{C}}$ spanned by ${ }_{5} \mathrm{~A}$ and ${ }_{5} \mathrm{C}$ combined.

In discussing the transformation functions for this case, it is convenient to define a vector $g_{j m}$ in $\vec{r}_{1}$ space whose representative $g_{j m}\left(\vec{r}_{1}\right)=\left(\vec{r}_{1} \mid g_{j m}\right)$ is the overlap integral (eq. (7)). The transformation function (eq. (67)) and its adjoint represent, respectively, the operators $K_{2}(m j)=1_{r_{0}}\left(g_{j m} \mid\right.$ and $\left.K_{2}(m j)^{\dagger}=1_{r_{0}} \mid g_{j m}\right)$. The iterates

$$
\begin{aligned}
& \mathrm{K}^{\prime}\left(\overrightarrow{\mathrm{r}}_{0}, \overrightarrow{\mathrm{r}}_{0^{\prime}} ; \mathrm{mm}^{\prime}\right)=\left\langle\overrightarrow{\mathrm{r}}_{0}, \phi_{\mathrm{m}}\left|\mathrm{\Pi}_{\mathrm{C}}\right| \overrightarrow{\mathrm{r}}_{0^{\prime}}, \phi_{\mathrm{m}^{\prime}}\right\rangle \\
& \overline{\mathrm{K}}^{\prime}\left(\overrightarrow{\mathrm{r}}_{0}, \overrightarrow{\mathrm{r}}_{1} ; \overrightarrow{\mathrm{r}}_{0^{\prime}}, \overrightarrow{\mathrm{r}}_{1^{\prime}} ; \mathrm{j}^{\prime}\right)=\left\langle\overrightarrow{\mathrm{r}}_{0}, \overrightarrow{\mathrm{r}}_{1}, \psi_{\mathrm{j}}\left|\Pi_{\mathrm{A}}\right| \overrightarrow{\mathrm{r}}_{0^{\prime}}, \overrightarrow{\mathrm{r}}_{1^{\prime}}, \psi_{\mathrm{j}^{\prime}}\right\rangle
\end{aligned}
$$

correspond to the respective operators

$$
\left.\begin{array}{l}
K^{\prime}\left(m, m^{\prime}\right)=\Sigma_{j} K_{2}(m j) K_{2}\left(m^{\prime} j\right)^{\dagger}=1_{r_{0}} c_{m m^{\prime}} \\
\left.j^{\prime}\right)=\Sigma_{m} K_{2}\left(m j^{\dagger}{ }^{\dagger} K_{2}\left(m j^{\prime}\right)=1_{r_{0}} \Sigma_{m} \mid g_{j m}\right)\left(g_{j^{\prime} m} \mid\right.
\end{array}\right\}
$$

where the constants

$$
c_{\mathrm{mm}}=\Sigma_{\mathrm{j}}\left(\mathrm{g}_{\mathrm{jm}} \mid \mathrm{g}_{\mathrm{jm}}{ }^{\prime}\right)
$$

constitute a Hermitian M-dimensional matrix. The $\overline{\mathbf{K}}^{\prime}\left(\mathrm{j}, \mathrm{j}^{\prime}\right)$ form an $\mathrm{N}^{\prime}$-dimensional array of operators in $\left(\vec{r}_{0}, \vec{r}_{1}\right)$ space, where $N^{\prime}$ is the number of $j$ values contained in the set $C$. Note that $K^{\prime}\left(m, m^{\prime}\right)^{\dagger}=K^{\prime}\left(m^{\prime}, m\right)$ and $\bar{K}^{\prime}\left(j, j^{\prime}\right)^{\dagger}=\bar{K}^{\prime}\left(j^{\prime}, j\right)$. 
The required operator products are

$$
\begin{aligned}
& \Pi_{\mathrm{A}} \Pi_{\mathrm{C}}=\Sigma_{\mathrm{m}} \Sigma_{\mathrm{j}} \int \mathrm{d} \overrightarrow{\mathrm{r}}_{0^{\prime}}\left|\overrightarrow{\mathrm{r}}_{0^{\prime}}, \phi_{\mathrm{m}}\right\rangle \mathrm{K}_{2}\left(\overrightarrow{\mathrm{r}}_{0^{\prime}}, \overrightarrow{\mathrm{r}}_{0}{ }^{\prime \prime}, \overrightarrow{\mathrm{r}}_{1}{ }^{\prime \prime} ; \mathrm{mj}\right)\left\langle\overrightarrow{\mathrm{r}}_{0^{\prime \prime}}, \overrightarrow{\mathrm{r}}_{1}{ }^{\prime \prime}, \psi_{\mathrm{j}}\right| \mathrm{d} \overrightarrow{\mathrm{r}}_{0}{ }^{\prime \prime} \mathrm{d} \overrightarrow{\mathrm{r}}_{1}{ }^{\prime \prime} \\
& \left.=1_{r_{0}} \Sigma_{m^{j}} \Sigma_{j} \mid \phi_{m}\right)\left(g_{j m} \psi_{j} \mid\right. \\
& \left.\Pi_{A} \Pi_{C} \Pi_{A}=\Sigma_{m} \Sigma_{m^{\prime}} K^{\prime}\left(m, m^{\prime}\right) \mid \phi_{m}\right)\left(\phi_{m^{\prime}} \mid\right. \\
& \left.\Pi_{C} \Pi_{A} \Pi_{C}=\Sigma_{j} \Sigma_{j} \bar{K}^{*}\left(j, j^{\prime}\right) \mid \psi_{j}\right)\left(\psi_{j} \mid\right.
\end{aligned}
$$

The operational rules are conveniently summarized as follows:

$$
\begin{gathered}
\Pi_{\alpha m^{\prime}}\left(\rho \phi_{m}\right)=\delta_{m^{\prime} m^{\prime}} \rho \phi_{m} \\
\Pi_{\alpha m}\left(\zeta \psi_{j}\right)=\left[K_{2}(m j) \zeta\right] \phi_{m} \\
\Pi_{c j}\left(\rho \phi_{m}\right)=\left[K_{2}(m j)^{\dagger} \rho\right] \psi_{j} \\
\Pi_{c j^{\prime}}\left(\zeta \psi_{j}\right)=\delta_{j^{\prime} j} \zeta \psi_{j}
\end{gathered}
$$

A basis for $\varsigma_{\mathrm{cj}}$ is given by the following construction. Consider the set of $\mathrm{M}$ vectors $\left\{g_{j m}, m \subset A\right\}$ in $\vec{r}_{1}$ space. From these vectors construct an orthonormal set $\mathrm{w}_{\mathrm{j}} \bar{\nu}$. On the assumption that the $\mathrm{g}_{\mathrm{jm}}$ are independent, the new set also contains $M$ menbers and the index $\bar{\nu}$ can be taken to have the values $\bar{\nu}=1,2, \ldots, M$. Now suppose that the set $w_{j} \nu$, of which the first $M$ members are the $w_{j \bar{\nu}}$, is a complete orthonormal set for functions of $\overrightarrow{\mathrm{r}}_{1}$. The vectors $\mathrm{w}_{\mathrm{j} \dot{\nu},}, \dot{\nu} \geq \mathrm{M}+1$, are orthogonal to the original $\mathrm{g}_{\mathrm{jm}}$, since the latter vectors are linear combinations of the $\mathrm{w}_{\mathrm{j} \bar{\nu}}$ only. The vectors $w_{j} i$ that complete the set are not unique, of course. The constants are defined as

$$
\mathrm{b}_{\mathrm{m}, j \nu}=\left(\mathrm{g}_{\mathrm{jm}} \mid \mathrm{w}_{\mathrm{j} \nu}\right)=\left(\phi_{\mathrm{m}} \mid \mathrm{w}_{\mathrm{j} \nu} \psi_{\mathrm{j}}\right)
$$

with $\mathrm{b}_{\mathrm{m}, \mathrm{j} \dot{\nu}=0}$ and $\mathrm{g}_{\mathrm{jm}}=\Sigma \Sigma_{\bar{\nu}} \mathrm{b} \mathrm{m}, \mathrm{j} \bar{\nu}^{*} \mathrm{w}_{\mathrm{j}} \bar{\nu}$. It will be convenient to use the index $\kappa$ to specify the pairs $(j, \bar{\nu})$, which number $\bar{M}=N^{\prime} M$. The constants $b_{m, j} \equiv b_{m, \kappa}$ form an $\mathbf{M} \times \overline{\mathrm{M}}$ matrix b. Directly from equation (116), $\mathbf{c}_{\mathrm{mm}^{\prime}}=\left(\mathrm{bb}^{\dagger}\right)_{\mathrm{mm}}$, and from equation (115), 


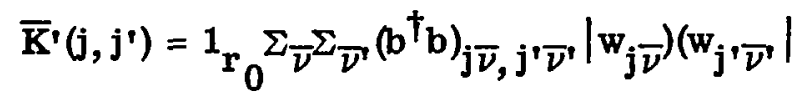

Thus, in $\vec{r}_{1}$ space, $\overline{\mathbf{K}}^{\prime}\left(\mathbf{j}, \mathbf{j}^{\prime}\right)$ operates only in the subspace spanned by the set $\left\{\mathbf{w}_{\mathbf{j}} \bar{\nu}\right\}$ and transforms to a vector in the subspace spanned by the set $\left\{w_{j} \bar{\nu}\right\}$. Finally, the desired orthonormal basis vectors spanning $\mathfrak{S}_{\mathrm{cj}}$ are $\mathbf{z}_{\mu, \mathrm{j} \nu}=\mathbf{x}_{\mu} \mathbf{w}_{j} \psi_{j}$.

The projector $\Pi_{\mathrm{A}+\mathrm{C}}$ can now be easily constructed by the methods that have been used previously from the forms

$$
\begin{gathered}
\Pi_{A+C}=\Pi_{A}+\Pi_{C p A} \\
\Pi_{A+C}=\Pi_{C}+\Pi_{A p C} \\
\Pi_{A+C}=R_{A}+R_{C}
\end{gathered}
$$

where

$$
\left.\begin{array}{c}
R_{A^{\prime}} \Psi=\Sigma_{m} F_{m}^{\prime} \phi_{m} \\
R_{C} \Psi=\Sigma_{j} T_{j} \psi_{j}
\end{array}\right\}
$$

and $F_{m}, T_{j}$ are, respectively, vectors in $\vec{r}_{0}$ space and $\left(\vec{r}_{0}, \vec{r}_{1}\right)$ space. The form (eq. (118)) leads to the examination of the vectors

$$
\begin{aligned}
\overline{\mathbf{z}}_{\mu, \mathrm{j} \nu} & =\left(1-\Pi_{\mathrm{A}}\right) \mathbf{z}_{\mu, \mathrm{j} \nu} \\
& =\mathbf{x}_{\mu} \mathbf{w}_{\mathbf{j} \nu} \psi_{\mathbf{j}}-\mathbf{x}_{\mu} \Sigma_{\mathbf{m}^{\mathbf{b}}, \mathbf{j} \nu} \phi_{\mathrm{m}}
\end{aligned}
$$

which separate naturally into two subsets, namely,

$$
\left.\begin{array}{c}
\overline{\mathbf{z}}_{\mu, \mathrm{j} \bar{\nu}}=\mathbf{x}_{\mu} \varphi_{\mathrm{j} \bar{\nu}} \\
\overline{\mathrm{z}}_{\mu, \mathrm{j} \dot{\nu}}=\mathbf{z}_{\mu, \mathrm{j} \dot{\nu}}
\end{array}\right\}
$$

where 


$$
\varphi_{\mathrm{j} \nu}=\mathrm{w}_{\mathrm{j}} \bar{\nu}_{\mathrm{j}}-\Sigma_{\mathrm{m}} \mathrm{b}_{\mathrm{m}, \mathrm{j}} \bar{\nu}_{\mathrm{m}}
$$

The subspace ${ }^{S_{C p A}}$ is spanned by the totality of vectors $\overline{\mathbf{z}}_{\mu, \mathrm{j} \nu}$ given by equation (122). Because of their factors $\mathbf{X}_{\mu}$, any two vectors having different values of $\mu$ are orthogonal. Also, any vector $\overline{\mathrm{z}}_{\mu, \mathrm{j} \dot{\nu}}$ is orthorgonal to every other vector. Thus, to obtain an orthonormal basis that spans ${ }_{5} \mathrm{CpA}$, construct, for each value of $\mu$, an orthonormal set from the $\overline{\mathbf{M}}$ vectors $\mathrm{z}_{\mu, \mathrm{j} \bar{\nu}} \equiv \mathrm{z}_{\mu, \kappa}$. The scaler products

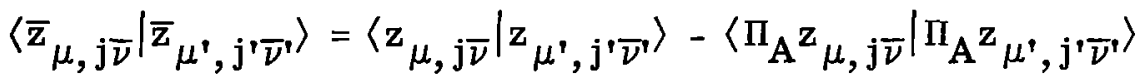

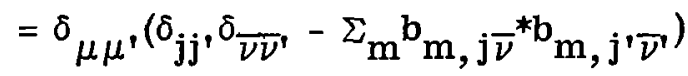

$$
\begin{aligned}
& =\delta_{\mu \mu r}\left[1 \overline{\mathbf{M}}-\mathrm{b}^{\dagger} \mathrm{b}\right]_{\kappa \kappa^{\prime}}
\end{aligned}
$$

suggests introducing the Hermitian matrix $\bar{a}=1_{\bar{M}}-b^{\dagger} b$ which is associated with the matrix $a=1_{M}-b^{\dagger}$ by the relations $a b=b \bar{a}$ and $\bar{a}^{-1}=1 \bar{M}+b^{\dagger} a^{-1} b$. A unitary matrix $U$ that diagonalizes $\overline{\mathrm{a}}$ as

$$
\mathrm{U}^{\dagger} \overline{\mathrm{a}} \mathrm{U}=\overline{\mathrm{a}}_{\mathrm{D}}=\left[\overline{\mathrm{a}}_{\kappa} \delta_{\kappa K^{\prime}}\right]
$$

gives the orthogonal vectors

$$
\mathrm{Z}_{\mu \kappa}=\mathrm{N}_{\kappa^{\prime} \Sigma^{\prime}} \mathrm{U}_{\kappa^{\prime} \kappa^{\prime}} \overline{\mathrm{z}}_{\mu \kappa^{\prime}}
$$

It follows from equations (124) and (125) that

$$
\left\langle\mathrm{Z}_{\mu \kappa} \mid \mathrm{Z}_{\mu^{\prime} \kappa^{\prime}}\right\rangle=\mathrm{N}_{\kappa^{\prime} \overline{\mathrm{a}}^{\delta}{ }^{\delta} \mu^{\prime}{ }^{\delta}{ }_{\kappa \kappa^{\prime}}},
$$

The normalization of the vectors (eq. (126)) is accomplished by the choice $N_{K}=\left(\bar{a}_{K}\right)^{-1 / 2}$.

The set of vectors made up of the totality of the $\mathrm{z}_{\mu_{K}}$ and the $\bar{z}_{\mu, j \dot{\nu}}$ is an orthonormal basis for ${ }^{5} \mathrm{CpA}$. The projector for this subspace is

$$
\begin{aligned}
& \Pi_{\mathrm{CpA}}=\Sigma_{\mu}\left[\Sigma_{\kappa}\left|\mathrm{z}_{\mu \kappa}\right\rangle\left\langle\mathrm{z}_{\mu \kappa}\left|+\Sigma_{\mathrm{j}} \Sigma_{\dot{\nu}}\right| \mathrm{z}_{\mu, \mathrm{j} \dot{\nu}}\right\rangle\left\langle\mathrm{z}_{\mu, \mathrm{j} \dot{\nu}}\right|\right]
\end{aligned}
$$

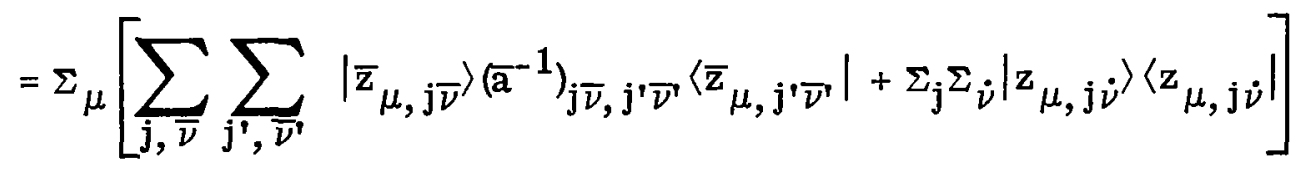


where the relation $\bar{a}^{-1}=U\left(\bar{a}_{D}\right)^{-1} U^{\dagger}$ has been used. When the second sum on the right of equation (127) is augmented by the terms required to extend the summation over all values of the indices, the result is simply $\Pi_{C}$ :

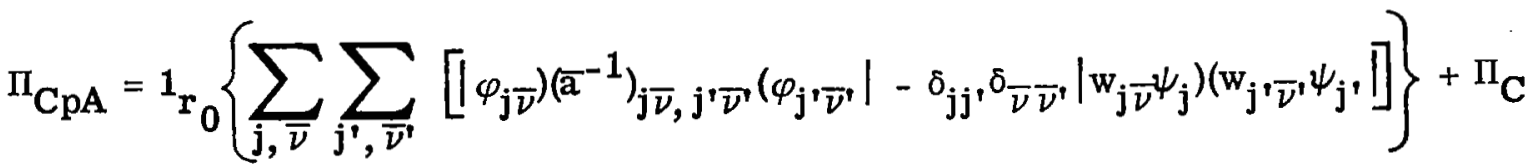

Equation (127) can also be written as

$$
\Pi_{\mathrm{CpA}}=\left(1-\Pi_{\mathrm{A}}\right) \bar{\Lambda}^{\prime}\left(1-\Pi_{\mathbf{A}}\right)
$$

where

$$
\begin{aligned}
& \bar{\Lambda}^{\prime}=\sum_{\mu} \sum_{\mathrm{j}, \bar{\nu}} \sum_{\mathrm{j}^{\prime}, \bar{\nu}^{\prime}} \mid \mathrm{z}_{\mu, \mathrm{j} \bar{\nu}}\left(\overline{\mathrm{a}}^{-1}-1_{\overline{\mathrm{M}}}\right)_{\mathrm{j} \bar{\nu}, \mathrm{j}^{\prime} \bar{\nu}^{\prime}}\left\langle\mathrm{z}_{\mu, \mathrm{j}^{\prime} \bar{\nu}^{\prime}}\right|+\Pi_{\mathbf{C}} \\
& \left.=\Sigma_{\mathbf{j}} \Sigma_{\mathbf{j}^{\prime}} \overline{L^{\prime}}\left(\mathbf{j j ^ { \prime }}\right) \mid \psi_{\mathbf{j}}\right)\left(\psi_{\mathbf{j}^{\prime}}\right)
\end{aligned}
$$

and

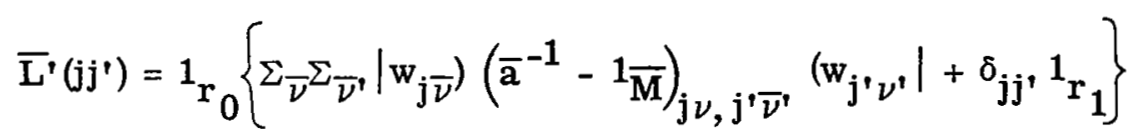

The form (eq. (120)) is obtained from the conditions

$$
\begin{aligned}
& \Pi_{A}\left(1-\Pi_{C}\right)\left(1-\Pi_{A+C}\right)=0 \\
& \Pi_{C}\left(1-\Pi_{A}\right)\left(1-\Pi_{A+C}\right)=0
\end{aligned}
$$

which give

$$
\begin{aligned}
R_{A^{\prime}} & =\Pi_{A} \Lambda^{\prime} \Pi_{A}\left(1-\Pi_{C}\right) \\
R_{C} & =\Pi_{C} \bar{\Lambda}^{\prime} \Pi_{C}\left(1-\Pi_{A}\right)
\end{aligned}
$$


where $\Lambda^{\prime}$ and $\bar{\Lambda}^{\prime}$ are, respectively, operators in $\mathfrak{S}_{\mathrm{A}}$ and $\mathfrak{S}_{\mathrm{C}}$ defined by the relations

$$
\left.\begin{array}{l}
\Pi_{A} \Lambda^{\prime} \Pi_{A}\left(1-\Pi_{C}\right) \Pi_{A}=\Pi_{A} \\
\Pi_{C} \bar{\Lambda}^{\prime} \Pi_{C}\left(1-\Pi_{A}\right) \Pi_{C}=\Pi_{C}
\end{array}\right\}
$$

Inserting the forms

$$
\left.\Lambda^{\prime}=\Sigma_{m^{\prime}} \Sigma_{m^{\prime}} L^{\prime}\left(m^{\prime}\right) \mid \phi_{m}\right)\left(\phi_{m^{\prime}} \mid\right.
$$

and

$$
\left.\overline{\Lambda^{\prime}}=\Sigma_{\mathbf{j}} \Sigma_{\mathbf{j}^{\prime}}, \bar{L}^{\prime}\left(\mathrm{jj}^{\prime}\right) \mid \psi_{\mathbf{j}}\right)\left(\psi_{\mathbf{j}^{\prime}} \mid\right.
$$

into the defining equations (eqs. (131)) yields

$$
L^{\prime}\left(1_{r_{0}}{ }^{1} M-1_{r_{0}} b^{\dagger}\right)=1_{r_{0}}{ }^{1} M
$$

and

$$
\Sigma_{j^{\prime}, \bar{L}^{\prime}\left(j j^{\prime \prime}\right)}\left[{ }_{\mathrm{r}_{0}} \delta_{\mathrm{j}^{\prime \prime} \mathrm{j}^{\prime}}-\overline{\mathrm{K}}^{\prime}\left(\mathrm{j}^{\prime \prime}, \mathrm{j}^{\prime}\right)\right]={ }_{\mathrm{r}_{0}}{ }^{1} \mathrm{r}_{1}{ }^{1} \overline{\mathrm{N}}
$$

The solution of equation (134) is $L^{\prime}=1_{r_{0}} a^{-1}$, and it is easily verified that the solution of equation (135) is given by equation (130). The operators (eqs. (129) and (133)) are identical, so that $\Pi_{\mathrm{CpA}}=\left(1-\Pi_{\mathrm{A}}\right) \mathrm{R}_{\mathrm{C}}$. It is evident from equations (128) and (130) that the vectors $\mathrm{w}_{\dot{\nu}}, \dot{\nu} \geq \overline{\mathrm{N}}+1$, have been eliminated in the final forms of $\Pi_{\mathrm{A}+\mathrm{C}}$ by the closure property.

\section{COUPLED EQUATIONS AND THEIR BOUNDARY CONDITIONS}

\section{Asymptotic Properties}

The operators developed in the preceding sections act on the total scattering state $\Psi$ to produce components whose coordinate representations yield asymptotically the transition amplitudes for the reaction in the various channels. The projector $\Pi_{\alpha \mathrm{m}}$, for 
example, operates on $\Psi$ to produce a component $U_{m}\left(\vec{r}_{0}\right) \phi_{m}$, given by equation (6), which at large values of $r_{0}$ has the behavior of the $\alpha \mathrm{m}$ term on the right of equation (1), if channel $\alpha \mathrm{m}$ is open. Of course, $\mathrm{U}_{\mathrm{m}}\left(\mathrm{r}_{0}\right)$ becomes vanishing for closed channels. Similarly, the component $V_{n}(\vec{R})(\chi \psi)_{n}$ projected out by $\Pi_{\beta n}$ has the asymptotic behavior given by equation (2). The corresponding properties of the components produced by the projectors for the redefined channel subspaces are illustrated by the examples of $\Pi_{\beta^{\prime} n}$ and $\Pi_{\bar{\beta} n}$, which are defined by equations (43) and (99), respectively.

Write

$$
\left.\begin{array}{l}
\Pi_{\beta^{\prime} \mathbf{n}} \Psi=Y_{n}(\chi \psi)_{n} \\
\Pi_{\bar{\beta} \mathbf{n}} \Psi=\bar{Y}_{n}(\eta \psi)_{n}
\end{array}\right\}
$$

where

$$
\mathrm{Y}_{\mathrm{n}}\left(\overrightarrow{\mathrm{r}}_{0}\right)=\left\langle\overrightarrow{\mathrm{r}}_{0},(\chi \psi)_{\mathrm{n}} \mid \Psi\right\rangle
$$

and

$$
\overline{\mathrm{Y}}_{\mathbf{n}}\left(\overrightarrow{\mathrm{r}}_{0}\right)=\left\langle\overrightarrow{\mathrm{r}}_{0},(\eta \psi)_{\mathbf{n}} \mid \Psi\right\rangle
$$

The integrations in the scalar products can be performed with the volume element $\mathrm{d} \tau=\mathrm{d} \overrightarrow{\mathrm{r}}_{0} \mathrm{~d} \overrightarrow{\mathrm{r}} \mathrm{d} \vec{\xi}$, in which case the suitable wave function representative is $\left\langle\overrightarrow{\mathrm{r}}_{0}, \overrightarrow{\mathrm{r}}, \xi \mid \Psi\right\rangle$. The asymptotic behavior in the $\beta$ channels of the wave function based on the coordinate pair $\left(\vec{r}_{0}, \vec{r}\right)$ is obtained from the expressions

$$
\begin{gathered}
R=\left(\mathrm{r}_{0}^{2}-\overrightarrow{\mathrm{r}}_{0} \cdot \overrightarrow{\mathrm{r}}+\frac{1}{4} \mathrm{r}^{2}\right)^{1 / 2}=\mathrm{r}_{0}-\frac{1}{2} \hat{\mathrm{r}}_{0} \cdot \overrightarrow{\mathrm{r}}+O\left(\frac{\mathrm{r}^{2}}{\mathrm{r}_{0}}\right) \\
\hat{\mathrm{R}}=\frac{1}{\mathrm{R}}\left(\overrightarrow{\mathrm{r}}_{0}-\frac{1}{2} \overrightarrow{\mathrm{r}}\right)
\end{gathered}
$$

which show that, for large separations in the $\beta$ channel,

$$
\mathrm{R}_{\vec{\beta}} \mathbf{r}_{0}-\frac{1}{2} \hat{\mathbf{r}}_{0} \cdot \overrightarrow{\mathbf{r}} \quad \hat{\mathbf{R}}_{\vec{\beta}} \hat{\mathbf{r}}_{0}
$$


From equation (2),

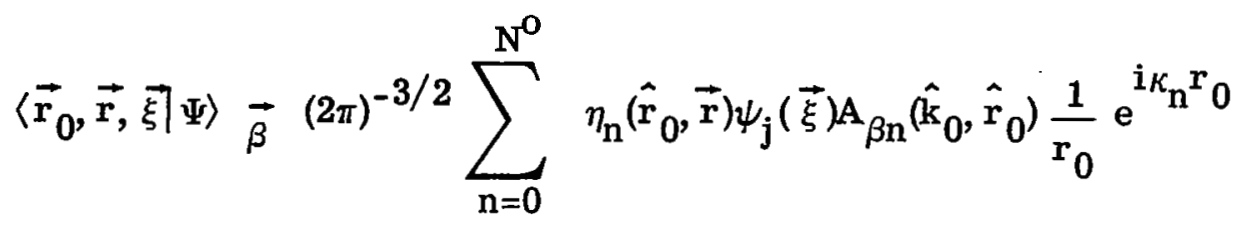

where $\eta_{\mathrm{n}}\left(\hat{\mathrm{r}}_{0}, \overrightarrow{\mathrm{r}}\right)$ is given by equation (98). The a symptotic behavior of $\mathrm{Y}_{\mathrm{n}}\left(\overrightarrow{\mathrm{r}}_{0}\right)$ and $\overline{\mathrm{Y}}_{\mathbf{n}}\left(\overrightarrow{\mathrm{r}}_{0}\right)$ follows directly:

$$
\begin{aligned}
& \mathrm{Y}_{\mathrm{n}}\left(\mathrm{r}_{0}\right) \vec{\beta}(2 \pi)^{-3 / 2} \sum_{\mathrm{n}^{\prime}=0}^{N^{O}} \bar{\nu}_{\mathrm{nn}},\left(\hat{\mathrm{r}}_{0}\right) \mathrm{A}_{\beta \mathrm{n}^{\prime}},\left(\hat{\mathrm{k}}_{0}, \hat{\mathrm{r}}_{0}\right) \frac{1}{\mathrm{r}_{0}} \mathrm{e}^{\mathrm{i} \kappa_{\mathrm{n}^{\prime}}, \mathrm{r}_{0}} \\
& \left.\overline{\mathrm{Y}}_{\mathrm{n}}\left(\mathrm{r}_{0}\right) \rightarrow(2 \pi)^{-3 / 2} \sum_{\mathrm{n}^{\prime}=0}^{N^{0}} \nu_{\mathrm{nn}},\left(\hat{\mathrm{r}}_{0}\right) \mathrm{A}_{\beta \mathrm{n}^{\prime}}, \hat{\mathrm{k}}_{0}, \hat{\mathrm{r}}_{0}\right) \frac{1}{\mathrm{r}_{0}} \mathrm{e}^{\mathrm{i} \kappa_{\mathrm{n}^{\prime}}, \mathrm{r}_{0}}
\end{aligned}
$$

The functions

$$
\bar{\nu}_{\mathrm{nn}^{\prime}}\left(\hat{\mathrm{r}}_{0}\right)=\delta_{\mathrm{jj}}, \int \chi_{\mathrm{i}}^{*}\left(\overrightarrow{\mathrm{r}}^{\prime}\right) \exp \left(\frac{-\mathrm{i} \kappa_{\mathrm{n}^{\prime}}, \hat{\mathrm{r}}_{0} \cdot \overrightarrow{\mathrm{r}}^{\prime}}{2}\right) \chi_{\mathbf{i}^{\prime}}\left(\overrightarrow{\mathrm{r}}^{\prime}\right) \mathrm{d} \overrightarrow{\mathrm{r}}^{\prime}
$$

are closely related to the functions $\nu_{n n},\left(\hat{\mathrm{r}}_{0}\right)$ given by equation (100). Equation (137) shows that, in terms of the coordinate pair $\left(\vec{r}_{0}, \vec{r}\right)$, the wave function at large separations in the $\beta$ channels is a sum of functions that are not orthogonal on the scalar product over the internal variables of the $\beta$ fragments. Each term in the sum contributes to the projection in a given redefined $\beta$ channel. The phase factor in $\eta_{n}$, which multiplies a spherical wave on the right of equation (137), depends on the coordinate $\vec{r}$ as well as $\hat{\mathrm{r}}_{0}$ and shows explicitly the nonuniform nature of the asymptotic motion in the $\beta$ channels when described by the coordinate pair $\left(\overrightarrow{\mathrm{r}}_{0}, \overrightarrow{\mathrm{r}}\right)$.

The projection operator (eq. (66)) produces the component

$$
\Pi_{c j} \Psi=w_{j} \psi_{j}
$$


where $W_{j}$ is a vector in $(\vec{R}, \vec{r})$ space, or equivalently, in $\left(\vec{r}_{0}, \vec{r}_{1}\right)$ space. The expansion

$$
w_{j}(\vec{R}, \vec{r}) \equiv\left\langle\vec{R}, \vec{r} \mid w_{j}\right\rangle=\Sigma_{i} a_{i}(\vec{R}) X_{i}(\vec{r})
$$

is convenient for discussing the asymptotic properties of $W_{j}(\vec{R}, \vec{r})$. The sum on the right of equation (141) includes the continuum states of $\mathscr{D}$ as well as the bound states. At large separations in the $\beta$ channels, that is, for large $\mathrm{R}$ and $\mathrm{r} \lesssim \mathrm{R}_{\mathrm{D}}$, the discrete terms in the sum have the asymptotic behavior given by equation (2). In contrast to the components (eq. (136)), which refer to specific $\beta$ channels, the component (eq. (140)) does not vanish at asymptotic values of the variables in the open channel $\alpha \mathrm{m}$. This asymptotic contribution in channel $\alpha \mathrm{m}$ corresponds to the projection of $\phi_{\mathrm{m}}$ on $\psi_{\mathrm{j}}$ and is described by a superposition of continuum terms in the sum (eq. (141)). The contribution is missing in the term $\Pi_{\mathrm{ApC}} \Psi$ from equation (119), and therefore this term does not yield the complete asymptotic behavior in the $\alpha$ channels. If the three particle channels $(n, p$, and $d-1)$ are open, there will be additional nonvanishing continuum components $a_{i}(\vec{R})$, at large values of $R$, corresponding to the appropriate continuum states of $\mathscr{P}$.

The coupled equations describing the reaction can be formulated in terms of the components $F_{m}$ and $G_{n}$ of equation (81), $\bar{F}_{m}$ and $\bar{G}_{n}$ of equation (103), or $F_{m}$ and $\mathrm{T}_{\mathrm{j}}$ of equation (121). The asymptotic properties of the component functions provide the corresponding boundary conditions that define the desired solutions of the system of equations. From the conditions analogous to equations (10) and (11), namely,

$$
\left.\begin{array}{l}
\Pi_{\alpha \mathrm{m}}\left(1-\Pi_{\mathrm{A}+\mathrm{B}^{\prime}}\right)=0 \\
\Pi_{\beta^{\prime} \mathrm{n}}\left(1-\Pi_{\mathrm{A}+\mathrm{B}^{\prime}}\right)=0
\end{array}\right\}
$$

are obtained, with the help of the operator rules (eq. (87)),

$$
\begin{gathered}
U_{m}=F_{m}+\Sigma_{n} J_{1}(m n) G_{n} \\
Y_{n}=\Sigma_{m} J_{1}(m n)^{\dagger} F_{m}+G_{n}
\end{gathered}
$$

From the property (eq. (48)) on the matrix elements (eq. (83)), it follows that $F_{m}\left(\vec{r}_{0}\right)-U_{m}\left(\vec{r}_{0}\right)$ and $G_{n}\left(\vec{r}_{0}\right)-Y_{n}\left(\vec{r}_{0}\right)$. The functions $F_{m}\left(\vec{r}_{0}\right)$ for the $\alpha$ channels have the usual outgoing and incident wave behavior given by equation (1). However, the functions for the $\beta$ channels require that the boundary condition of outgoing waves only be 
imposed on appropriate linear combinations, namely,

$$
\sum_{n^{\prime}=0}^{N^{0}}\left[\bar{\nu}^{-1}\left(\hat{r}_{0}\right)\right]_{n n^{\prime}} G_{n^{\prime}}\left(\vec{r}_{0}\right) \rightarrow(2 \pi)^{-3 / 2} A_{\beta n}\left(\hat{k}_{0}, \hat{r}_{0}\right) \frac{1}{r_{0}} e^{i \kappa_{n} x_{0}}
$$

When the equations analogous to equation (142) for $\Pi_{A+\bar{B}}$ (with $\Pi_{\bar{\beta} n}$ replacing $\Pi_{\beta^{\prime} n}$ ) are employed, and the vanishing of the matrix elements (eq. (104)) at large $r_{0}$ is taken into account,

$$
\begin{aligned}
& \overline{\mathbf{F}}_{\mathrm{m}}\left(\overrightarrow{\mathrm{r}}_{0}\right)-\mathrm{U}_{\mathrm{m}}\left(\overrightarrow{\mathrm{r}}_{0}\right) \\
& \overline{\mathrm{G}}_{\mathrm{n}}\left(\overrightarrow{\mathrm{r}}_{0}\right) \rightarrow \sum_{\mathrm{n}^{\prime}=0}^{N^{O}}\left[\nu^{-1}\left(\hat{\mathrm{r}}_{0}\right)\right]_{\mathrm{n} n^{\prime}} \overline{\mathrm{Y}}_{\mathrm{n}^{,}}\left(\overrightarrow{\mathrm{r}}_{0}\right) \\
& \rightarrow(2 \pi)^{-3 / 2} A_{\beta n}\left(\hat{k}_{0}, \hat{r}_{0}\right) \frac{1}{r_{0}} e^{i \kappa_{n} r_{0}}
\end{aligned}
$$

Hence, the outgoing wave boundary condition applies directly to the functions $\overline{\mathrm{G}}_{\mathrm{n}}\left(\overrightarrow{\mathrm{r}}_{0}\right)$. The analogs of equation (142) for $\Pi_{A+C}$ (with $\Pi_{c j}$ appearing in place of $\Pi_{\beta^{\prime} n}$ ) give

$$
\begin{aligned}
U_{m} & =F_{m}{ }^{\prime}+\Sigma_{j} K_{2}(m j) T_{j} \\
& =F_{m}{ }^{\prime}+\Sigma_{j}\left(\phi_{m} \mid T_{j} \psi_{j}\right) \\
W_{j} & =\Sigma_{m} F_{m}{ }^{\prime} g_{j m}+T_{j}
\end{aligned}
$$

Asymptotically in the $\beta$ channels, both $r_{0}$ and $r_{1}$ become large, and equation (145) shows that

$$
\mathrm{T}_{\mathbf{j}}\left(\overrightarrow{\mathrm{r}}_{0}, \overrightarrow{\mathrm{r}}_{1}\right) \vec{\beta} \mathrm{W}_{\mathrm{j}}\left(\overrightarrow{\mathrm{r}}_{0}, \overrightarrow{\mathrm{r}}_{1}\right)
$$

In the $\alpha$ channels, however, $r_{1}$ remains bounded as $r_{0}$ becomes large, and 


$$
\begin{aligned}
& F_{m}{ }^{\prime}\left(r_{0}\right)+\left(\vec{r}_{0}, g_{j m} \mid T_{j}\right) \vec{\alpha}_{m} U_{m_{0}}\left(\vec{r}_{0}\right) \\
& \Sigma_{m} F_{m}\left(\vec{r}_{0}\right) g_{j m}\left(\vec{r}_{1}\right)+T_{j}\left(\vec{r}_{0}, \vec{r}_{1}\right) \vec{\alpha}_{j} W_{j}\left(\vec{r}_{0}, \vec{r}_{1}\right)
\end{aligned}
$$

\section{Coupled Equations}

In the method given by Feshbach (ref. 1), the Schrodinger equation $(\mathrm{E}-\mathscr{H}) \Psi=0$ yields an equation for $P \Psi$

$$
(\mathrm{E}-\mathrm{H}) \mathrm{P} \Psi=0
$$

in which the effective Hamiltonian is

$$
\mathrm{H}=\mathscr{H}_{\mathrm{PP}}+\mathscr{H}_{\mathrm{PQ}} \frac{1}{\mathrm{E}-\mathscr{H}_{\mathrm{QQ}}} \mathscr{x}_{\mathrm{QP}}
$$

Here, $\mathrm{P}$ is the open channel projection operator, $\mathrm{Q}=1-\mathrm{P}$, and $\mathscr{H}_{\mathrm{PQ}} \equiv \mathrm{P} \mathscr{} \mathrm{Q}$, and so forth. In the case of the pickup reaction, $P$ can be chosen as one of the projectors in the section MULTICHANNEL PROJECTION OPERATORS, where all the open channels are included in the sets $\mathrm{A}$ and $\mathrm{B}$. If there are open three-particle channels, the projector $\Pi_{\mathrm{A}+\mathrm{C}}$ would be required.

The projector $\Pi_{A+B}$, in the form of equation (80), for example, leads to a set of coupled equations for the functions $F_{m}\left(\vec{r}_{0}\right)$ and $G_{n}\left(\vec{r}_{0}\right)$. Operating on equation (147) with $\Pi_{\alpha \mathrm{m}}$ and $\mathrm{II}_{\beta^{\prime} n}$, respectively, gives

$$
\left.\begin{array}{r}
E\left[F_{m}+\sum_{n=0}^{N^{O}} J_{1}(m n) G_{n}\right]=\left(\phi_{m}|H| \widetilde{R}_{A} \Psi+R_{B^{\prime}} \Psi\right\rangle \\
E\left[\sum_{m=0}^{M^{o}} J_{1}(m n)^{\dagger} F_{m}+G_{n}\right]=\left([\chi \psi]_{n}|H| \widetilde{R}_{A} \Psi+R_{B^{\prime}} \Psi\right\rangle
\end{array}\right\}
$$

The boundary conditions for the $F_{m}$ are given by equation (1) and for the $G_{n}$ by equation (143). The form of $\Pi_{A+B}$, given by equations (78) and (94) produces channel components which are all orthogonal to one another. The resulting coupled equations are analogous to equation (149), but they have a more convenient form with only one channel 
function appearing on the left side of each equation. On the other hand, the unitary matrix $U_{n^{\prime} n}\left(\vec{r}_{0}\right)$ in equation (92) must be evaluated in this approach.

An appropriate separation of the total Hamiltonian is associated with each type of channel. The separation corresponding to the $\alpha$ channels is $\mathscr{H}=\mathrm{T}_{\alpha}+\mathscr{X}_{\alpha}+\mathrm{V}_{\alpha}$, where $\mathrm{V}_{\alpha}$ is the interaction between the $\alpha$ fragments, $\mathrm{T}_{\alpha}$ is the sum of their center-of-mass kinetic energies, and $\mathscr{x}_{\alpha}$ is the sum of their internal Hamiltonians (with each internal Hamiltonian referred to its fragment's own center-of-mass system). There is a similar resolution for the $\beta$ channels. Recall that the projector $\Pi_{A+B^{\prime}}$ is based on the choice of natural coordinates for the $\alpha$ channels. Consequently, the usual form of $\mathrm{T}_{\alpha}$ suffices for the partial wave expansion of the functions $F_{m}\left(\vec{r}_{0}\right)$. The kinetic energy operator $T_{\beta}$, however, must be expressed in a form suitable to the choice of coordinates $\left(\vec{r}_{0}, \vec{r}\right)$ in the $\beta$ channels. Since the core nucleus $\alpha-1$ is infinitely massive, the reference frame provided by the fixed core itself has been used. In this reference frame, $T_{\beta}=\left(1 / 2 \mathrm{M}_{D}\right) \overrightarrow{\mathrm{P}}_{\beta}^{2}$, where $\overrightarrow{\mathrm{P}}_{\beta}$ is the total momentum of the fragment $\mathscr{D}$, and $\mathrm{M}_{\mathrm{D}}=\mathrm{M}_{\mathrm{n}}+\mathrm{M}_{\mathrm{p}}$ is its mass. For simplicitly, the masses of $\mathrm{n}$ and $\mathrm{p}$ have been taken to be the same, namely $M$, so that their total mass is $M_{D}=2 M$ and their reduced mass is $\mu=(1 / 2) M$. The kinetic energy $T_{\beta}$ is the same as that of a point mass $M_{D}$ whose velocity is that of the center of mass of fragment $\mathscr{D}$. Thus, the following equation can be written:

$$
T_{\beta}=\frac{1}{2} M_{D}\left(\frac{d \vec{R}}{d t}\right)^{2}=\frac{1}{2} M_{D}\left(\frac{d}{d t} \vec{r}_{0}-\frac{d}{d t} \frac{1}{2} \vec{r}\right)^{2}
$$

The form (eq. (150)) gives the transformation of the kinetic energy from a reference frame moving with velocity $\left(\mathrm{d} \vec{r}_{\sigma} / d t\right)$, in which particle $n$ is at rest, to the reference frame of the fixed core. The term

$$
\frac{1}{2} M_{D}\left(\frac{d \frac{1}{2} \vec{r}}{d t}\right)^{2}
$$

is the kinetic energy of the point mass $M_{D}$ in the moving reference frame. The quantities $\overrightarrow{\mathbf{P}}_{\mathrm{n}}=\mathbf{M}_{\mathrm{n}}\left(\mathrm{d} \overrightarrow{\mathbf{r}}_{0} / \mathrm{dt}\right)$ and $\mathrm{p}=\mu(\mathrm{d} \overrightarrow{\mathrm{r}} / \mathrm{dt})$ are the momentum of particle $\mathrm{n}$ in the frame of the fixed core and in the center-of-mass system of fragment $\mathscr{B}$, respectively. The kinetic energy operator becomes

$$
T_{\beta}=\frac{1}{2} M_{D}\left(\frac{\vec{P}_{n}}{M_{n}}-\frac{\vec{p}}{2 \mu}\right)^{2}=M^{-1}\left(\vec{P}_{n}-\vec{p}\right)^{2}
$$


The momentum operators have the coordinate representations $\overrightarrow{\mathrm{P}}_{\mathrm{n}} \rightarrow-i \hbar \nabla_{\mathbf{r}_{0}}$ and $\vec{p} \rightarrow-i \hbar \nabla_{\mathbf{r}}$. Finally,

$$
\mathbf{T}_{\beta}=\frac{1}{\mathbf{M}}\left[-\frac{\hbar^{2}}{\mathbf{r}_{0}} \frac{\partial}{\partial \mathbf{r}_{0}}\left(\frac{\partial}{\partial \mathbf{r}_{0}} \mathbf{r}_{0}\right)+\frac{1}{\mathbf{r}_{0}^{2}} \overrightarrow{\mathrm{L}}^{2}+2 \hbar^{2} \nabla_{\mathbf{r}_{0}} \cdot \nabla_{\mathbf{r}}-\hbar^{2} \nabla_{\mathbf{r}}^{2}\right]
$$

where $\overrightarrow{\mathrm{L}}=\overrightarrow{\mathbf{r}}_{0} \times \overrightarrow{\mathrm{P}}_{\mathrm{n}}$. The terms arising from $\mathrm{T}_{\beta}$ in the coupled equations (eqs. (149)) are much less complicated than the corresponding contribution in the coupled equations given by $\Pi_{\mathrm{A}+\overline{\mathrm{B}}}$.

Consider now a selected set of open $\alpha$ and $\beta$ channels, for which an appropriate projector is $\Pi_{M}$, of the kind given in the section MULTICHANNEL PROJECTION OPERATORS, for example. Then, an open-channel subspace $\mathscr{P}$ of the class that has been used here can be resolved into the direct sum of ${ }_{5}$, the projective space of $\Pi_{M}$, and of $\mathfrak{S}_{\mathrm{K}}$, the largest orthogonal subspace in $\mathscr{P}$. Thus,

$$
\left.\begin{array}{c}
\mathscr{P}=\varsigma_{M} \oplus \varsigma_{K} \\
P=\Pi_{M}+\Pi_{K} \\
\Pi_{M^{M}} \Pi_{K}=0
\end{array}\right\}
$$

With the same procedure as was used in reference 7 for the case of a selected set of inelastic channels, the generalized potential for the projection $\Pi_{M} \Psi$ can be derived. The steps involved are exactly analogous to those which lead from equation (40) to equation (45) in reference 7. In the present case, start with equation (151) and replace $\Pi_{I}$ by $\Pi_{M}$ in the subsequent equations of reference 7 . In terms of the generalized potential, the components of $\Pi_{M} \Psi$ satisfy a set of coupled equations. Appropriate energy averaging of the transition amplitude leads to an expression for the generalized optical potential that describes the direct reaction in the selected channels. Again, the procedure directly parallels the derivation in reference 7 for the case of inelastic scattering.

The optical model Hamiltonian can be written

$$
h_{M}=h^{(+)}+u_{C N}
$$

The compound-nucleus term $\mathrm{u}_{\mathrm{CN}}$ gives the energy-averaged resonance contribution to the transition amplitude. The scattering eigenstate $\theta^{(+)}$of $h_{M}$ yields the energy- 
averaged transition amplitudes in the selected channels. Necessary conditions are

$$
\begin{aligned}
& \Pi_{\mathbf{M}^{\theta^{(+)}}=\theta^{(+)}} \\
& \Pi_{M^{h}}{ }^{M}=h_{M} \Pi_{M}=h_{M}
\end{aligned}
$$

If $\Pi_{M}$ is a projector of the type $\Pi_{A+B^{\prime}}$, coupled equations for $\Pi_{A+B^{\prime}}{ }^{(+)}$follow in the same way as equation (149), except that $h_{M}$ appears in place of $\mathrm{H}$, and $\Pi_{A+B}$, now refers to a selected set of open $\alpha$ and $\beta$ channels. If a phenomenological optical potential is associated with the exact potential given by $h_{M}$, the condition (eq. (153)) can be taken to hold for the solution $\bar{\theta}^{(+)}$given by the empirical generalized potential, that is, $\bar{\Pi}_{\mathbf{M}} \bar{\theta}^{(+)}=\bar{\theta}^{(+)}$. Here, $\bar{\Pi}_{\mathbf{M}}$ is the projector obtained by the replacement of the exact internal states in $\Pi_{M}$ by the approximate states given by the model. The appropriate $\bar{\Pi}_{M}$ is employed to obtain a set of coupled equations like equation (149) for the components of $\bar{\theta}^{(+)}$. The phenomenological potential consists of the various coefficient functions that enter in the coupled equations; it corresponds to the matrix elements of the associated $h_{M}$ taken between the internal states of the channels included in $\Pi_{M}$. The potential is determined by adjustment to produce the optimum fit to the experimental data.

Lewis Research Center,

National Aeronautics and Space Administration, Cleveland, Ohio, March 19, 1968, 129-02-07-07-22. 


\section{APPENDIX - SYMBOLS}

A selected set of $\alpha$ channels

$\mathrm{A}^{\mathrm{o}} \quad$ set of all open $\alpha$ channels

A $\alpha \mathrm{m} \quad$ transition amplitude for channel $\alpha \mathrm{m}$

$A_{\beta \mathbf{n}} \quad$ transition amplitude for channel $\beta \mathbf{n}$

nucleus consisting of particle $\mathrm{p}$ bound to the core

$\mathscr{A - 1}$ heavy core nucleus

a

matrix equal to $1_{M}-b b^{\dagger}$

$\vec{a}$ value of $\vec{r}_{0}$

$\bar{a} \quad$ matrix equal to $1_{\bar{M}}-b^{\dagger} b$

$\overline{\mathrm{a}}_{\mathrm{D}}$ diagonalized form of matrix $\overline{\mathrm{a}}$

$\bar{a}_{\kappa} \quad$ eigenvalue of matrix $\bar{a}$

B selected set of $\beta$ channels

B matrix equal to $1_{M}-\Delta \nu^{-1} \Delta^{\dagger}$

$B^{\circ} \quad$ set of all open $\beta$ channels

$\overline{\mathrm{B}}$ matrix equal to $1_{\mathrm{N}}-\nu^{-1} \Delta^{\dagger} \Delta$

b matrix whose elements are $b_{m}, \kappa$

$\mathrm{b}_{\mathrm{m}, \mathrm{j} \nu} \quad$ equal to $\quad\left(\mathrm{g}_{\mathrm{jm}} \mid \mathrm{w}_{\mathrm{j} \nu}\right)=\mathrm{b}_{\mathrm{m}, \kappa}$

$\vec{b} \quad$ value of $\vec{R}$

C selected set of $j$ values

C matrix equal to $1_{r_{0}} B^{-1}$

$\mathrm{C}_{\mathrm{mm}}$, matrix element of $\mathrm{C}$

C matrix equal to ${ }_{r_{0}}(\nu \bar{B})^{-1}$

$\overline{\mathrm{C}}_{\mathrm{nn}}$, matrix element of $\overline{\mathrm{C}}$

$c_{m m}$ equal to $\Sigma_{j}\left(g_{j m} \mid g_{j m}\right)$

$c_{o} \quad$ equal to $[(\mathrm{g} / \mathrm{g})]^{1 / 2}$

D subscript designating nucleus $\mathscr{P}$

9. bound system of particles $n$ and $p$ 


\begin{tabular}{|c|c|}
\hline d & $\mathbf{1}_{\mathbf{M}}-\mathbf{h h}^{\dagger}$ \\
\hline$\overline{\mathrm{d}}$ & $1_{N}-h^{\dagger} h$ \\
\hline$d \mathbf{v}$ & $d \vec{r}_{0} d \vec{r}_{1}$ \\
\hline $\mathrm{d} \tau$ & $d \vec{r}_{0} d \vec{r}_{1} d \vec{\xi}$ \\
\hline$d \tau_{1}$ & $\mathrm{~d} \vec{\rho}_{1} \mathrm{~d} \vec{r}_{1} \mathrm{~d} \vec{\xi}$ \\
\hline $\mathbf{E}$ & total energy \\
\hline $\mathbf{F}$ & vector in $\vec{R}$ space \\
\hline$F^{\prime}$ & vector in $\vec{r}_{0}$ space \\
\hline$\widetilde{\mathbf{F}}$ & vector in $\overrightarrow{\mathrm{r}}_{0}$ space \\
\hline $\mathbf{F}_{\mathbf{m}}^{\prime}$ & vector in $\overrightarrow{\mathbf{r}}_{0}$ space \\
\hline$\widetilde{\mathbf{F}}_{\mathrm{m}}$ & vector in $\vec{r}_{0}$ space \\
\hline$\overline{\mathrm{F}}_{\mathrm{m}}$ & vector in $\vec{r}_{0}$ space \\
\hline G & vector in $\vec{r}_{0}$ space \\
\hline$\widetilde{\mathrm{G}}$ & vector in $\vec{R}$ space \\
\hline $\mathrm{G}_{\mathrm{n}}$ & vector in $\vec{r}_{0}$ space \\
\hline$\overline{\mathrm{G}}_{\mathrm{n}}$ & vector in $r_{0}$ space \\
\hline$g\left(\vec{r}_{1}\right)$ & abbreviated rotation for $\mathrm{g}_{\mathrm{jm}}\left(\overrightarrow{\mathrm{r}}_{1}\right)$ \\
\hline $\mathrm{g}_{\mathrm{jm}}\left(\overrightarrow{\mathrm{r}}_{1}\right)$ & $\int \mathrm{d} \vec{\xi}^{\prime} \psi_{j}^{*}\left(\vec{\xi}^{\prime}\right) \phi_{\mathrm{m}}\left(\overrightarrow{\mathrm{r}}_{1}, \vec{\xi}_{1}^{\prime}\right)$ \\
\hline $\mathrm{H}$ & effective Hamiltonian \\
\hline $\mathscr{H}$ & total Hamiltonian of system \\
\hline $\mathscr{H} \mathbf{P Q}$ & $\mathbf{P} \mathscr{E Q}$ \\
\hline$\Phi_{\mathrm{A}}$ & direct sum of channel subspaces $\mathfrak{S}_{\alpha \mathrm{m}}, \mathrm{m} \subset \mathrm{A}$ \\
\hline$S_{A^{0}}$ & direct sum of all open channel subspaces $\S_{\alpha \mathrm{m}}$ \\
\hline $\mathfrak{S}_{\mathrm{B}}$ & direct sum of channel subspaces $\mathfrak{S}_{\beta n}, n \subset B$ \\
\hline $5_{\mathrm{B}^{\circ}}$ & direct sum of all open channel subspaces $\mathfrak{S}_{\beta \mathrm{n}}$ \\
\hline$S_{B^{\prime}}$ & direct sum of channel subspaces $\mathfrak{S}_{\beta^{\prime} \mathrm{n}}, \mathrm{n} \subset \mathrm{B}$ \\
\hline$\varsigma_{\overline{\mathrm{B}}}$ & space spanned by all subspaces $\aleph_{\bar{\beta} n}, n \subset B$ \\
\hline $5_{C}$ & direct sum of channel subspaces $\$_{c j}, j \subset C$ \\
\hline${ }^{5} \mathrm{~A}+\mathrm{B}$ & space spanned by $\mathfrak{S}_{\mathrm{A}}$ and $\mathfrak{5}_{\mathrm{B}^{\prime}}$ combined \\
\hline
\end{tabular}


$\mathfrak{S}_{\mathrm{B}^{\prime} \mathrm{pA}}$ largest subspace of $\mathfrak{S}_{\mathrm{B}^{\prime}}$, that is orthogonal to $\mathfrak{S}_{\mathrm{A}}$

$\mathfrak{S}_{\mathrm{ApB}}$ ' largest subspace of $\mathfrak{S}_{\mathrm{A}}$ that is orthogonal to $\mathfrak{S}_{\mathrm{B}}$ '

$\mathfrak{S}_{\mathrm{A}}+\overline{\mathrm{B}} \quad$ space spanned by $\mathfrak{S}_{\mathrm{A}}$ and $\mathfrak{S}_{\overline{\mathrm{B}}}$ combined

$\mathfrak{S}_{\overline{\mathrm{B}}} \mathrm{pA}$ largest subspace of $5_{\overline{\mathrm{B}}}$ that is orthogonal to ${ }^{5_{\mathrm{A}}}$

${ }^{S_{A p} \bar{B}}$ largest subspace of $\mathscr{S}_{\mathrm{A}}$ that is orthogonal to $\mathfrak{S}_{\overline{\mathrm{B}}}$

$\aleph_{\mathrm{A}+\mathrm{C}} \quad$ space spanned by $\aleph_{\mathrm{A}}$ and $\varsigma_{\mathrm{C}}$ combined

${ }^{5_{\mathrm{CpA}}}$ largest subspace of ${ }^{\Phi_{\mathrm{C}}}$ that is orthogonal to ${ }^{\mathfrak{S}_{\mathrm{A}}}$

${ }^{5} \mathrm{ApC}$ largest subspace of $\sqrt{5}_{\mathrm{A}}$ that is orthogonal to ${ }^{\mathfrak{s}_{\mathrm{C}}}$

${ }^{5} \mathbf{K}$

$\aleph_{\mathbf{M}}$

the largest subspace of $\mathscr{P}$ that is orthogonal to ${ }_{5} \mathrm{M}$

${ }^{5} \alpha$

${ }^{5} \alpha^{\prime}$

${ }^{5} \alpha \mathrm{m}$

the projective space of $\Pi_{M}$

${ }^{5} \alpha^{\prime} m$

abbreviated notation for $\varsigma_{\alpha \mathrm{m}}$

$\mathfrak{5}_{\beta}$

abbreviated notation for ${ }^{5} \alpha^{\prime} \mathrm{m}$

${ }^{5} \beta \mathrm{n}$

$s_{\beta}^{\prime n}$

channel subspace spanned by all states representable by $\rho\left(\overrightarrow{\mathrm{r}}_{0}\right) \phi_{\mathrm{m}}\left(\overrightarrow{\mathrm{r}_{1}}, \vec{\xi}\right)$

$5 \bar{\beta} \mathrm{n}$

$5_{\mathrm{cj}}$

$S_{c}$

$S_{\alpha+\beta}$

channel subspace spanned by all states representable by $s(\vec{R}) \phi_{m}\left(\vec{r}_{1}, \vec{\xi}\right)$

abbreviated notation for $\S_{\beta n}$

$\varsigma_{\beta p} \alpha$

channel subspace spanned by all states representable by $\sigma(\overrightarrow{\mathrm{R}})(\chi \psi)_{\mathrm{n}}$

channel subspace spanned by all states representable by $t\left(\vec{r}_{0}\right) \chi_{i}(\vec{r}) \psi_{j}(\vec{\xi})$

subspace spanned by all vectors representable by $\rho\left(\overrightarrow{\mathbf{r}}_{0}\right)(\eta \psi)_{\mathrm{n}}$

$5_{\alpha \mathrm{p} \beta}$

channel subspace spanned by all states representable by $\zeta(\vec{R}, \vec{r}) \psi_{\mathbf{j}}(\vec{\xi})$

abbreviated notation for $\varsigma_{c j}$

$5_{\alpha+\beta}$,

space spanned by $\mathfrak{S}_{\alpha}$ and $\mathfrak{S}_{\beta}$ combined

$5_{\beta^{\prime} \mathrm{p} \alpha}$

largest subspace of $\mathfrak{s}_{\beta}$ that is orthogonal to $\mathfrak{s}_{\alpha}$

largest subspace of $\mathfrak{S}_{\alpha}$ that is orthogonal to $\mathfrak{S}_{\beta}$

space spanned by $\mathfrak{S}_{\alpha}$ and $\mathfrak{5}_{\beta}$, combined

$5_{\alpha \mathrm{p} \beta^{\prime}}$

largest subspace of $\mathfrak{5}_{\beta}$, that is orthogonal to $\mathfrak{s}_{\alpha}$

$5^{5} \alpha^{+}+\beta$

largest subspace of $\mathfrak{S}_{\alpha}$ that is orthogonal to $\mathfrak{S}_{\beta}$,

$5 \alpha^{\prime} \mathrm{p} \beta$

space spanned by $\mathfrak{S}_{\alpha}$, and $\S_{\beta}$ combined

$5_{\beta p \alpha^{\prime}}$

largest subspace of $\mathfrak{S}_{\alpha^{\prime}}$ that is orthogonal to $\mathfrak{S}_{\beta}$

largest subspace of $\mathfrak{S}_{\beta}$ that is orthogonal to ${ }^{5} \alpha^{\text {, }}$ 


$$
\begin{aligned}
& 5_{\alpha+c} \quad \text { space spanned by } \mathscr{S}_{\alpha} \text { and } 5_{c} \text { combined } \\
& 5_{\alpha \mathrm{pc}} \\
& 5_{\mathrm{cp} \alpha} \\
& \mathrm{h}\left(\overrightarrow{\mathrm{r}}_{\mathbf{0}}\right) \\
& \mathrm{h}_{\mathrm{mn}}\left(\overrightarrow{\mathrm{r}}_{0}\right) \\
& \mathbf{h}\left(\overrightarrow{\mathbf{r}}_{0}\right) \\
& \mathrm{h}_{\mathrm{D}}\left(\overrightarrow{\mathrm{r}}_{0}\right) \\
& h_{n} \\
& \mathbf{h}_{\mathbf{M}} \\
& \mathbf{h}^{(+)} \\
& \text {I } \\
& \overline{\mathbf{I}} \\
& I_{1}\left(\vec{R}, \vec{R}^{\prime}\right) \\
& \mathrm{I}_{1} \\
& \text { i } \\
& \text { J } \\
& \bar{J} \\
& \mathrm{~J}_{1}\left(\overrightarrow{\mathrm{r}}_{0}, \overrightarrow{\mathrm{r}}_{0}^{\prime}\right) \\
& \mathrm{J}_{1}\left(\overrightarrow{\mathrm{r}}_{0}, \overrightarrow{\mathrm{r}}_{0^{\prime}} ; \mathrm{m}, \mathrm{n}\right) \\
& \mathrm{J}_{1} \\
& \mathrm{~J}_{1}(\mathrm{mn}) \\
& J\left(m, m^{\prime}\right) \\
& \text { largest subspace of } \mathfrak{S}_{\alpha} \text { that is orthogonal to } \mathfrak{s}_{\mathrm{c}} \\
& \text {-largest subspace of } \mathfrak{S}_{\mathrm{c}} \text { that is orthogonal to } \mathfrak{S}_{\alpha} \\
& \text { abbreviated notation for } \mathrm{h}_{\mathrm{mn}}\left(\overrightarrow{\mathrm{r}}_{0}\right) \\
& \int \mathrm{d} \overrightarrow{\mathbf{r}}_{1}{ }^{\prime} \mathrm{g}_{\mathrm{jm}} *\left(\overrightarrow{\mathrm{r}}_{1}{ }^{\prime}\right) \chi_{\mathbf{i}}\left(\overrightarrow{\mathbf{r}}_{0}-\overrightarrow{\mathbf{r}}_{1}{ }^{\prime}\right) \\
& \text { matrix whose elements are } h_{\mathrm{mn}}\left(\overrightarrow{\mathrm{r}}_{0}\right) \\
& \text { diagonalized form of matrix } h\left(\vec{r}_{0}\right) \\
& \text { an eigenvalue of } h \\
& \text { the exact optical model Hamiltonian } \\
& \text { direct reaction term of optical model Hamiltonian } h_{M} \\
& \mathrm{I}_{1} \mathrm{I}_{1}^{\dagger} \\
& \text { I } \\
& \langle\overrightarrow{\mathrm{R}}, \phi \mid \overrightarrow{\mathrm{R}}, \chi \psi\rangle \\
& \text { abbreviated notation for } I_{1}\left(\vec{R}, \vec{R}^{\prime}\right) \\
& \text { specifies state of nucleus } \mathscr{D} \\
& \mathrm{J}_{1} \mathrm{~J}_{1}^{\dagger} \\
& \mathrm{J}_{1}^{\dagger} \mathrm{J}_{1} \\
& \text { abbreviated notation for } \mathrm{J}_{1}\left(\overrightarrow{\mathrm{r}}_{0}, \overrightarrow{\mathrm{r}}_{0} ; \mathrm{m}, \mathrm{n}\right) \\
& \left\langle\overrightarrow{\mathbf{r}}_{0}, \phi_{\mathrm{m}} \mid \overrightarrow{\mathrm{r}}_{0},(\chi \psi)_{\mathrm{n}}\right\rangle \\
& \text { matrix of array } J_{1}(\mathrm{mn}) \\
& \text { array of operators represented in } \overrightarrow{\mathrm{r}}_{0} \text { space by } \mathrm{J}_{1}\left(\overrightarrow{\mathrm{r}}_{0}, \overrightarrow{\mathrm{r}}_{0} ; \mathrm{m}, \mathrm{n}\right) \\
& \sum_{n} J_{1}(m, n) J_{1}^{\dagger}\left(n, m^{\prime}\right) \\
& \bar{J}\left(n, n^{\prime}\right) \\
& \sum_{\mathrm{m}} J_{1}^{\dagger}(\mathrm{n}, \mathrm{m}) \mathrm{J}_{1}\left(\mathrm{~m}, \mathrm{n}^{\prime}\right) \\
& \text { Jacobian determinant } \\
& \text { Jacobian determinant } \\
& \text { specifies state of nucleus } \mathscr{A}-1 \\
& \mathrm{~K}_{1} \mathrm{~K}_{1}^{\dagger} \\
& \mathrm{K}_{1}^{\dagger} \mathrm{K}_{1}
\end{aligned}
$$




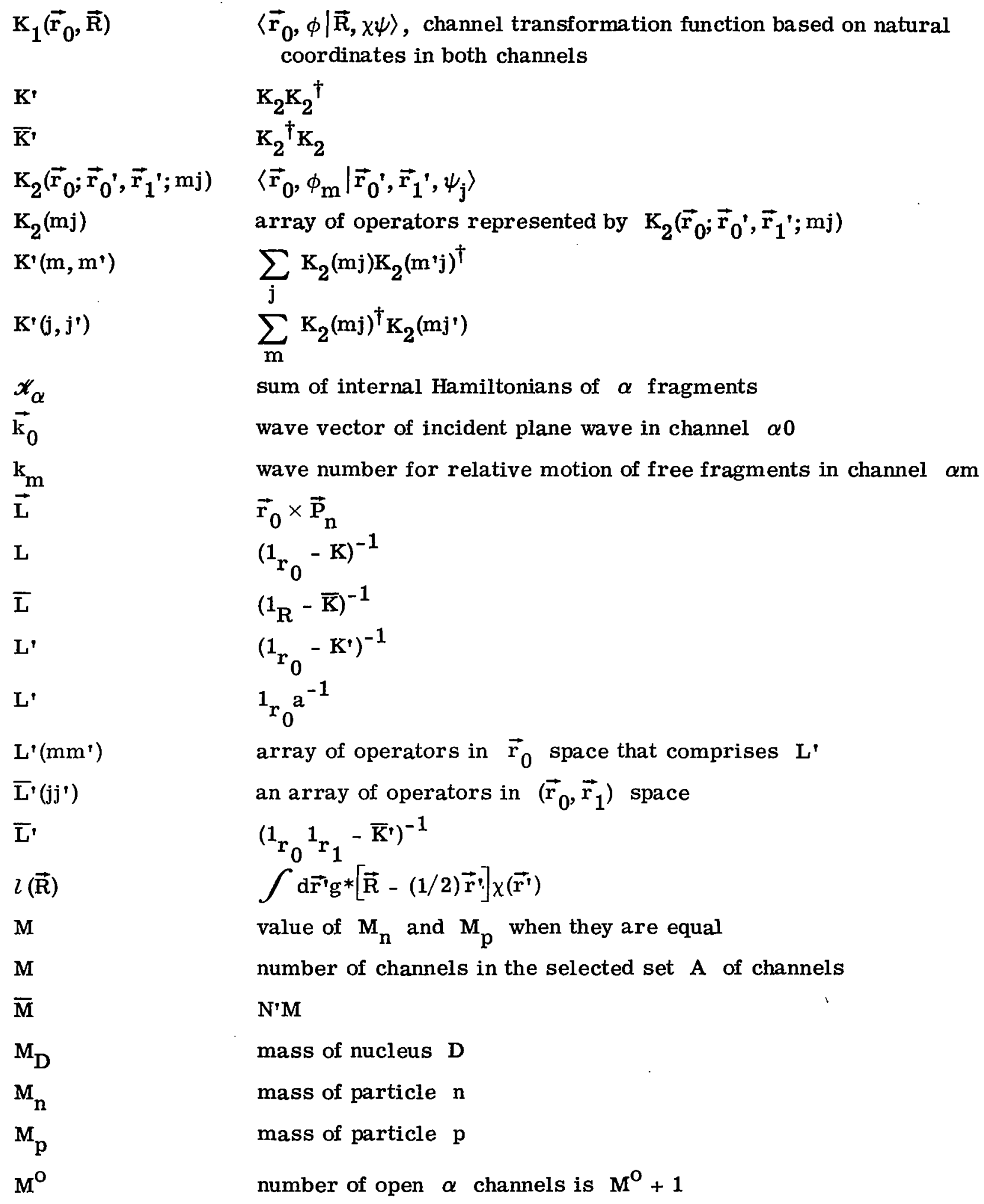




$$
\begin{aligned}
& M_{\lambda} \quad\left(1-\lambda^{-2}\right)^{-1 / 2} \\
& \text { m specifies state of nucleus } \mathscr{A} \\
& m(\vec{b}) \quad\left[1-(1 / 64)|l(\vec{b})|^{2}\right]^{-1 / 2} \\
& \mathrm{~N} \text { number of channels in selected set } \mathrm{B} \text { of } \beta \text { channels } \\
& \mathrm{N}^{\mathrm{O}} \quad \text { number of open } \beta \text { channels is } \mathrm{N}^{\mathrm{O}}+1 \\
& N \quad\left(1-c_{0}^{2}\right)^{-1 / 2} \\
& N^{r} \quad \text { number of } j \text { values in set } C \\
& N_{n}(\vec{a}) \quad\left[1-h_{n}(\vec{a})\right]^{-1 / 2} \\
& \overline{\mathrm{N}}_{\mathrm{n}}\left(\overrightarrow{\mathrm{r}}_{0}\right) \quad\left[\nu_{\mathrm{n}}\left(\hat{\mathrm{r}}_{0}\right)\right]^{-1 / 2} \\
& \mathrm{~N}_{\kappa} \quad\left(\bar{a}_{\kappa}\right)^{-1 / 2} \\
& \text { n specifies pair }(i, j) \\
& \text { n incident particle in pickup reaction } \\
& n(\vec{a}) \quad\left[1-|h(\vec{a})|^{2}\right]^{-1 / 2} \\
& 0 \quad \mathrm{~d}^{-1} \mathbf{1}_{\mathrm{r}_{0}} \\
& \sigma \quad \bar{d}^{-1}{ }^{1} r_{0} \\
& \mathrm{O}_{\mathrm{mm}} \text { ' matrix elements of } \mathrm{O} \\
& \overline{\mathrm{O}}_{\mathrm{nn}} \text { matrix elements of } \overline{\mathrm{O}} \\
& \mathbf{P} \quad \text { projection operator that selects all open channels } \\
& \overrightarrow{\mathrm{P}}_{\mathrm{n}} \quad \text { momentum of particle } \mathrm{n} \text { in frame of fixed core } \\
& \overrightarrow{\mathrm{P}}_{\beta} \quad \text { total momentum of fragment } \mathscr{D} \text { in frame of fixed core } \\
& \mathscr{P} \text { open channel subspace } \\
& \mathscr{T}_{\mathrm{M}} \quad \text { subspace of } \mathscr{P} \text { corresponding to Hilbert space of the model problem } \\
& \mathrm{p} \quad \text { particle bound to core } \mathscr{A}-1 \text { in nucleus } \& \\
& \vec{p} \quad \text { momentum of particle } n \text { in center-of-mass system of fragment } \mathscr{D} \\
& \text { Q } \quad \mathbf{1}-\mathbf{P} \\
& \mathbf{Q}_{\mathbf{S}} \quad \Pi_{\mathbf{M}}-\Pi_{\mathbf{S}} \\
& \overline{\mathbf{Q}}_{\mathbf{S}} \quad \text { counterpart of } \mathbf{Q}_{\mathbf{S}} \text { in model problem }
\end{aligned}
$$




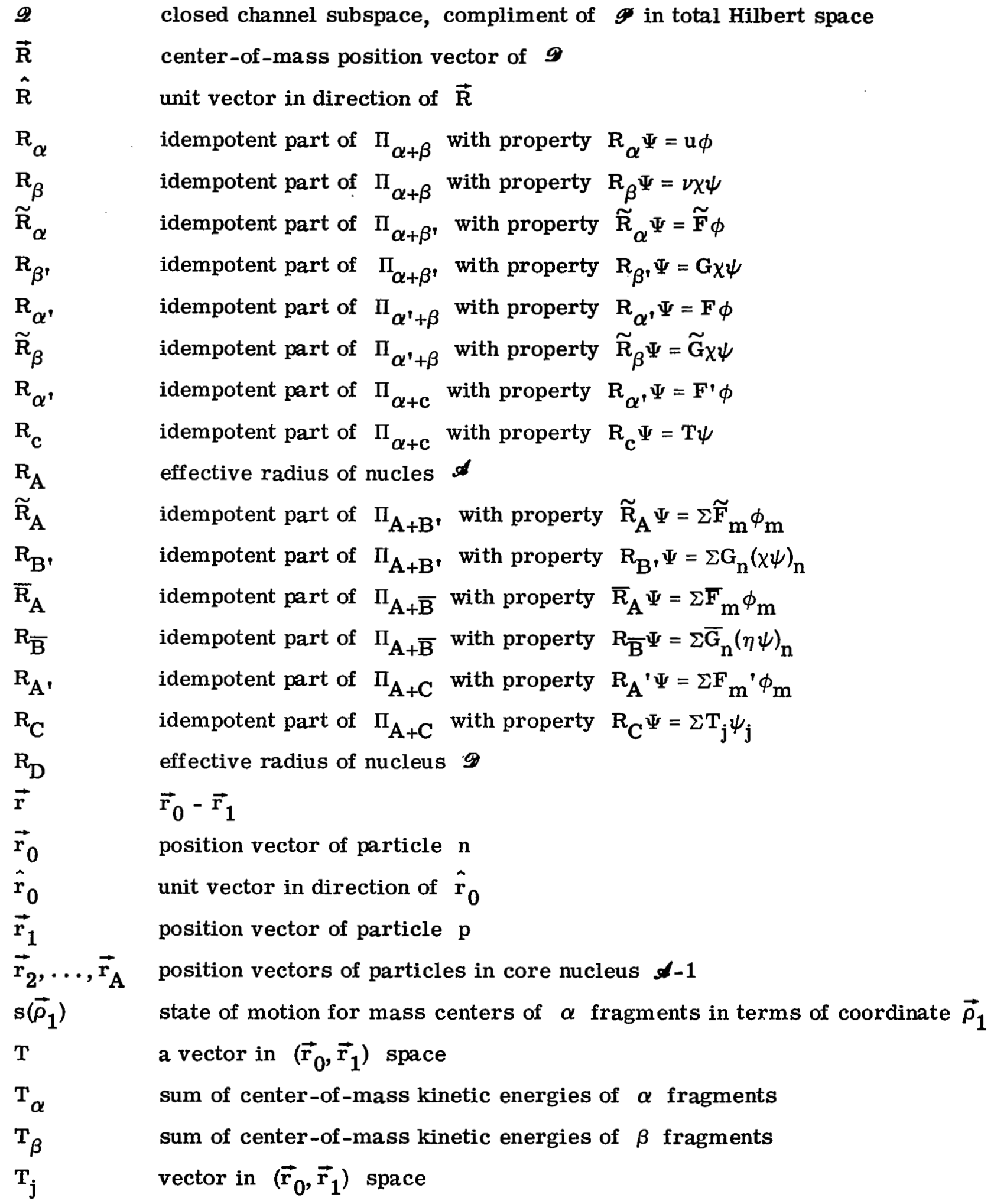


$\mathrm{t}\left(\vec{\rho}_{2}\right) \quad$ state of motion for mass centers of $\beta$ fragments in terms of coordinate $\vec{\rho}_{2}$

$\mathrm{U}$

$\mathrm{U}_{\mathrm{m}}\left(\overrightarrow{\mathrm{r}}_{0}\right)$

$\mathrm{u}$

$\mathbf{u}_{\lambda}\left(\overrightarrow{\mathrm{r}}_{0}\right)$

$\mathrm{u}_{\mathrm{CN}}$

$\mathrm{V}_{\mathrm{n}}(\overrightarrow{\mathrm{R}})$

$\mathrm{V}_{\alpha}$

$\mathbf{v}$

$\mathrm{v}_{\lambda}(\overrightarrow{\mathrm{R}})$

$\mathrm{w}_{\mathrm{j}}\left(\overrightarrow{\mathrm{r}}_{0}, \overrightarrow{\mathrm{r}}_{1}\right)$

$\mathrm{w}_{1}\left(\overrightarrow{\mathrm{r}}_{1}\right)$

$\mathrm{w}_{\nu}\left(\overrightarrow{\mathrm{r}}_{1}\right)$

$\mathrm{w}_{\mathrm{j} \bar{\nu}}\left(\overrightarrow{\mathrm{r}}_{1}\right)$

$\mathrm{w}_{\mathrm{j} \nu}\left(\overrightarrow{\mathrm{r}}_{1}\right)$

$\mathrm{x}_{\mu}\left(\overrightarrow{\mathrm{r}}_{0}\right)$

$\mathrm{x}_{\mathrm{b}}$

$\bar{x}_{b}$

$\overline{\mathbf{x}}_{\lambda}$

$\mathrm{Y}_{\text {an }}$

$\overline{\mathrm{Y}}_{\mathrm{r}_{0} \mathrm{n}}$

$\mathrm{Y}_{\mathrm{n}}\left(\overrightarrow{\mathrm{r}}_{0}\right)$

$\bar{Y}_{n}\left(\vec{x}_{0}\right)$

$\mathrm{y}_{\mathrm{a}}$

$\overline{\mathrm{y}}_{\mathrm{a}}$

$y_{\text {an }}$

$\overline{\mathrm{y}}_{\text {an }}$

$\mathrm{y}_{\lambda}$ unitary matrix

$\left\langle\overrightarrow{\mathrm{r}}_{0}, \phi_{\mathrm{m}} \mid \Psi\right\rangle$

vector in $\vec{r}_{0}$ space

eigenfunction of $\mathrm{K}$

compound-nucleus contribution to optical model Hamiltonian $h_{M}$

$\left\langle\overrightarrow{\mathrm{R}},(\chi \psi)_{\mathrm{n}} \mid \Psi\right\rangle$

iteraction between $\alpha$ fragments

vector in $\vec{R}$ space

eigenfunction of $\overline{\mathrm{K}}$

$\left\langle\overrightarrow{\mathrm{r}}_{0}, \overrightarrow{\mathrm{r}}_{1}, \psi_{\mathrm{j}} \mid \Psi\right\rangle$

$\left(1 / c_{0}\right) g\left(\vec{r}_{1}\right)$

complete orthonormal set for functions of $\vec{r}_{1}$

an orthonormal set constructed from the set $g_{j m}, m \subset A$

a complete orthonormal set whose first $M$ members are $w_{j}\left(\vec{r}_{1}\right)$

complete orthonormal set for functions of $\vec{r}_{0}$

$\delta_{b} \phi$

$\mathrm{m}(\overrightarrow{\mathrm{b}})\left(1-\Pi_{\beta}\right) \mathrm{x}_{\mathrm{b}}$

$\mathbf{M}_{\lambda}\left(1-\Pi_{\beta}\right) \mathrm{u}_{\lambda} \phi$

$\left.\delta_{a} \mid \Omega_{n}\right)$

$\overline{\mathrm{N}}_{\mathrm{n}}\left(\overrightarrow{\mathrm{r}}_{0}\right) \sum_{\mathrm{n}} \mu_{\mathrm{n}^{\prime} \mathrm{n}}\left(\hat{\mathrm{r}}_{0}\right)\left|\overrightarrow{\mathrm{r}}_{0},(\eta \psi)_{\mathrm{n}^{\prime}}\right\rangle$

$\left\langle\overrightarrow{\mathrm{r}}_{0},(\chi \psi)_{\mathrm{n}} \mid \Psi\right\rangle$

$\left\langle\vec{r}_{0},(\eta \psi)_{n} \mid \Psi\right\rangle$

$\delta_{\mathbf{a}} \times \psi$

$\mathrm{n}(\overrightarrow{\mathrm{a}})\left(1-\Pi_{\alpha}\right) \mathrm{y}_{\mathrm{a}}$

$\delta_{\mathrm{a}}(\chi \psi)_{\mathrm{n}}$

$\left(1-\Pi_{A}\right) y_{\text {an }}$

$\sigma_{\lambda} \times \psi$ 


\begin{tabular}{|c|c|}
\hline $\bar{y}_{\lambda}$ & $\mathrm{M}_{\lambda}\left(1-\Pi_{\alpha}\right) \mathrm{v}_{\lambda} \chi \psi$ \\
\hline$z_{\mu \kappa}$ & $\mathrm{N}_{\kappa^{\prime} \kappa^{\prime}} \mathrm{U}_{\kappa^{\prime} \kappa^{\prime}} \overline{\mathbf{z}}_{\mu \kappa^{\prime}}$ \\
\hline $\mathrm{z}_{\mu \nu}$ & $\mathbf{x}_{\mu} \mathbf{w}_{\nu} \psi$ \\
\hline$\overline{\mathrm{z}}_{\mu \nu}$ & $\left(1-\Pi_{\alpha}\right) \mathbf{z}_{\mu \nu}$ \\
\hline $\mathbf{z}_{\mu, \mathbf{j} \nu}$ & $\mathrm{x}_{\mu} \mathrm{w}_{\mathrm{j} \nu} \psi_{\mathrm{j}}$ \\
\hline$\overline{\mathbf{z}}_{\mu, j \nu}$ & $\left(1-\Pi_{\mathrm{A}}\right) \mathrm{z}_{\mu, j \nu}$ \\
\hline$\alpha$ & designates particles of certain kind of channel \\
\hline$\alpha \mathrm{m}$ & specifies channel of $\alpha$ type \\
\hline$\beta$ & designates particles of certain kind of channel \\
\hline$\beta \mathbf{n}$ & specifies channel of $\beta$ type \\
\hline$\Delta_{\mathrm{mn}}\left(\overrightarrow{\mathrm{r}}_{0}\right)$ & $\mathrm{d} \overrightarrow{\mathrm{r}}_{1}^{\prime} \mathrm{g}_{\mathrm{jm}} *\left(\overrightarrow{\mathrm{r}}_{1}^{\prime}\right) \eta_{\mathrm{n}}\left(\hat{\mathrm{r}}_{0}, \overrightarrow{\mathrm{r}}_{0}-\overrightarrow{\mathrm{r}}_{1}^{\prime}\right)$ \\
\hline$\delta_{\mathrm{a}}$ & normalized eigenvector of $J\left(m, m^{\prime}\right)$ and $\bar{J}\left(n, n^{\prime}\right)$ \\
\hline$\delta_{\mathrm{b}}$ & normalized eigenvector of $\mathrm{I}$ and $\overline{\mathbf{I}}$ \\
\hline$\zeta$ & vector in $(\vec{R}, \vec{r})$ space \\
\hline$\eta_{\mathrm{n}}\left(\hat{\mathrm{r}}_{0}, \overrightarrow{\mathrm{r}}\right)$ & $\exp \left(-\mathbf{i} \kappa_{\mathrm{n}} \hat{\mathrm{r}}_{0} \cdot \overrightarrow{\mathrm{r}} / 2\right) \chi_{\mathbf{i}}(\overrightarrow{\mathrm{r}})$ \\
\hline$\theta\left(\hat{\mathbf{r}}_{0}\right)$ & unitary matrix that diagonalizes $\nu\left(\hat{\mathbf{r}}_{0}\right)$ \\
\hline$\theta^{(+)}$ & scattering eigenstate of generalized optical potential $h_{M}$ \\
\hline $\bar{\theta}^{(+)}$ & scattering eigenstate of phenomenological potential associated with $h_{M}$ \\
\hline$\kappa$ & index that specifies pair $(j, \bar{\nu})$ \\
\hline$\kappa$ & constant \\
\hline$\kappa_{\mathrm{n}}$ & wave number for relative motion of free fragments in channel $\beta \mathrm{n}$ \\
\hline$\Lambda$ & $\mathrm{L} \mid \phi)(\phi \mid$ \\
\hline $\bar{\Lambda}$ & $\overline{\mathrm{L}} \mid \chi \psi)(x \psi \mid$ \\
\hline$\Lambda_{0}$ & $\mathrm{O} \mid \phi)(\phi \mid$ \\
\hline $\bar{\Lambda}_{\mathrm{O}}$ & $\overline{0} \mid x \psi)(x \psi \mid$ \\
\hline$\Lambda_{N}$ & $\mathrm{~m}^{2} 1_{\mathrm{R}^{\Pi}} \alpha_{\alpha^{\prime}}$ \\
\hline $\bar{\Lambda}_{N}$ & $\mathrm{~m}^{2} 1_{\mathrm{R}} \Pi_{\beta}$ \\
\hline$\Lambda^{\prime}$ & $\left.L^{\prime} \mid \phi\right)(\phi \mid$ \\
\hline $\bar{\lambda}$ & $\overline{\mathbf{L}} \mid \psi)(\psi \mid$ \\
\hline
\end{tabular}




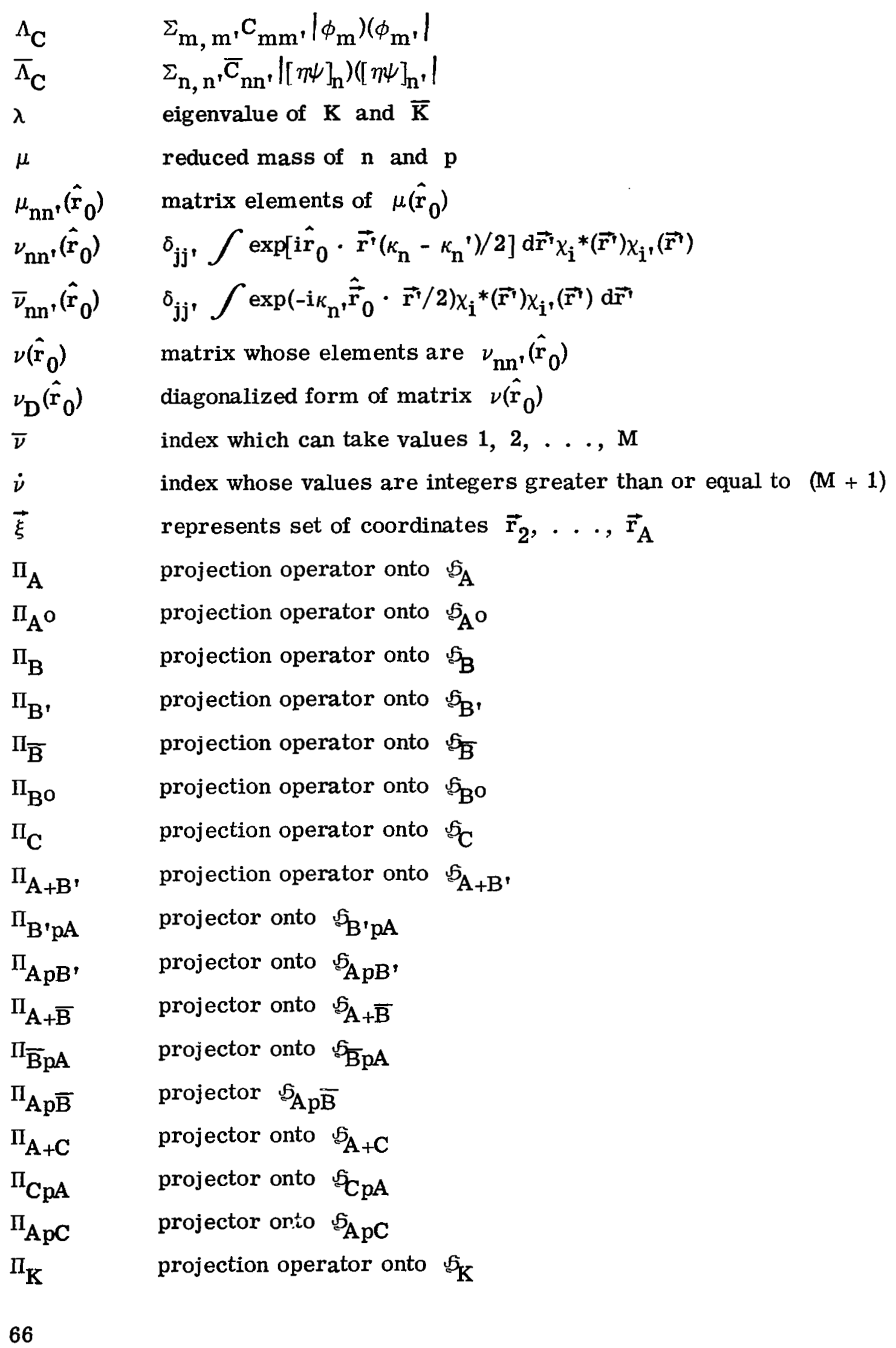


$\Pi_{\mathbf{M}}$

$\bar{\Pi}_{\mathbf{M}}$

$\Pi_{\mathrm{O}}$

$\Pi_{\mathbf{O}}$,

$\mathrm{II}_{\mathrm{S}}$

$\bar{\Pi}_{S}$

$\Pi_{\alpha}$

$\Pi_{\alpha \mathrm{m}}$

$\Pi_{\alpha^{\prime} \mathrm{m}}$

$\Pi_{\beta}$

$\Pi_{\beta \mathrm{n}}$

$\Pi_{\beta^{\prime} n}$

$\Pi_{c}$

$\Pi_{\mathrm{cj}}$

$\Pi_{\alpha+\beta}$

$\Pi_{\beta \mathrm{p} \alpha}$

$\mathrm{II}_{\alpha \mathrm{p} \beta}$

$\Pi_{\alpha+\beta}$,

$\Pi_{\beta^{\prime} \mathrm{p} \alpha}$

$\Pi_{\alpha \mathrm{p} \beta}$,

$\Pi_{\alpha^{\gamma}+\beta}$

$\Pi_{\alpha^{\prime} \mathrm{p} \beta}$

$\Pi_{\beta \mathrm{p} \alpha^{\prime}}$,

$\Pi_{\alpha+c}$

$\Pi_{\alpha \mathrm{pc}}$

$\Pi_{\mathrm{cp} \alpha}$

$\vec{\rho}_{1}$

$\vec{\rho}_{2}$

$\vec{\rho}$

$\rho\left(\overrightarrow{\mathrm{r}}_{0}\right)$ projection operator for a selected set of open channels

counterpart of $\Pi_{M}$ in model problem

projector onto largest subspace of that is orthogonal to $\mathscr{S}_{\mathrm{A}} \mathrm{o}$

projector onto largest subspace of $\mathscr{F}$ that is orthogonal to $F_{\mathrm{B}}$

alternative projection operator to $\Pi_{\mathbf{M}}$

counterpart of $\Pi_{S}$ in model problem

abbreviated notation for $\Pi_{\alpha}$

projection operator on $\mathfrak{5}_{\alpha \mathrm{m}}$

projection operator onto ${ }^{5} \alpha^{\prime} \mathrm{m}$

abbreviated notation for $\Pi_{\beta \mathrm{n}}$

projection operator onto $\Phi_{\beta} n$

projection operator onto $\mathfrak{S}_{\beta^{\prime} \mathrm{n}}$

abbreviated notation for $\Pi_{\mathbf{c j}}$

projector onto $\mathfrak{S}_{\mathbf{c j}}$

projector onto $\varsigma_{\alpha+\beta}$

projector onto $\mathfrak{S}_{\beta \mathrm{p} \alpha}$

projector onto $\S_{\alpha \mathrm{p} \beta}$

projector onto $\varsigma_{\alpha+\beta}$,

projector onto $\Im_{\beta^{\prime} p \alpha}$

projector onto ${ }^{5} \alpha \mathrm{p} \beta$,

projector onto $S_{\alpha^{+}+\beta}$

projector onto ${ }_{5}^{5} \alpha^{\prime} \mathrm{p} \beta$

projector onto $\Phi_{\beta p \alpha}$ '

projector onto $\mathfrak{S}_{\alpha+\mathrm{c}}$

projector onto $\S_{\alpha \mathrm{pc}}$

projector onto ${ }^{5} \mathrm{cp} \alpha$

coordinate for $\alpha$ channels, in addition to $\vec{r}_{1}$

coordinate for $\beta$ channels, in addition to $\mathbf{r}$

common coordinate for both $\alpha$ and $\beta$ channels

state of motion for mass centers of $\alpha$ fragments 


$$
\begin{aligned}
& \sigma(\vec{R}) \quad \text { state of motion for mass centers of } \beta \text { fragments } \\
& \sigma_{\lambda}(\vec{R}) \quad \text { complete orthonormal set for functions of } \vec{R} \\
& \phi_{\mathrm{m}}\left(\overrightarrow{\mathrm{r}}_{1}, \vec{\xi}\right) \quad \text { wave function for } \mathrm{m}^{\text {th }} \text { excited state of nucleus } A \\
& \phi\left(\overrightarrow{\mathrm{r}}_{1}, \vec{\xi}\right) \quad \text { abbreviated notation for } \phi_{\mathrm{m}}\left(\overrightarrow{\mathrm{r}}_{1}, \vec{\xi}\right) \\
& \varphi_{\mathrm{o}}^{\prime} \quad \mathrm{N}(\phi-\mathrm{g} \psi) \\
& \varphi_{0}^{\prime \prime} \quad \mathrm{N}\left(\mathrm{w}_{1} \psi-\mathrm{c}_{\mathrm{o}} \phi\right)
\end{aligned}
$$

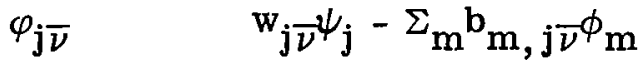

$$
\begin{aligned}
& \chi_{\mathbf{i}}(\overrightarrow{\mathrm{r}}) \quad \text { wave function for } i^{\text {th }} \text { excited state of particle } D \\
& \chi(\overrightarrow{\mathrm{r}}) \quad \text { abbreviated notation for } \chi_{\mathbf{i}}(\overrightarrow{\mathrm{r}}) \\
& {[\chi \psi]_{\mathrm{n}} \quad \chi_{\mathbf{i}} \psi_{\mathbf{j}}} \\
& \Psi \quad \text { total scattering state } \\
& \psi_{j}(\vec{\xi}) \quad \text { wave function for } j^{\text {th }} \text { excited state of nucleus } \mathscr{A}-1 \\
& \psi(\vec{\xi}) \quad \text { abbreviated notation for } \chi_{\mathrm{j}}(\vec{\xi}) \\
& \Omega_{\mathrm{n}} \quad \mathrm{N}_{\mathrm{n}} \Sigma_{\mathrm{n}^{\prime}}, \mathrm{U}_{\mathrm{n}^{\prime} \mathrm{n}}\left[(\chi \psi)_{\mathrm{n}^{\prime}}-\Sigma_{\mathrm{m}} \mathrm{h}_{\mathrm{mn}}, \phi_{\mathrm{m}}\right] \\
& \omega_{0} \quad \mathrm{n}(\chi \psi-\mathrm{h} \phi) \\
& \omega_{0}^{\prime} \quad \mathrm{m}[\phi-(1 / 8) l * \chi \psi] \\
& \omega_{\mathrm{n}} \quad(\chi \psi)_{\mathrm{n}}-\Sigma_{\mathrm{m}} \mathrm{h}_{\mathrm{mn}} \phi_{\mathrm{m}}
\end{aligned}
$$

Mathematical notations:

$\hbar \quad$ Planck's constant divided by $2 \pi$

$\delta_{\mathrm{mm}} \quad \quad$ Kronecker delta

$\nabla_{\mathbf{r}} \quad$ gradient operator with respect to coordinate $\overrightarrow{\mathbf{r}}$

$\langle\mid\rangle \quad$ scalar product over a complete set of variables for system

(|) scalar product over a set of variables that is not a complete set for system

$1_{r} \quad$ unit vector in $\vec{r}$ space

$1_{M} \quad$ M-dimensional unit matrix

$\oplus \quad$ direct sum

* complex conjugate

$\dagger \quad$ Hermitian adjoint

c is contained in 
$\cap \quad$ intersection

$\left\{x_{\mu}\right\} \quad$ the set $x_{\mu}$ 


\section{REFERENCES}

1. Feshbach, Herman: A Unified Theory of Nuclear Reactions. II. Ann. Phys. (N.Y.), vol. 19, no. 2, Aug. 1962, pp. 287-313.

2. Mittleman, Marvin H. : Coupled Equations for Rearrangement Collisions. Ann. Phys. (N.Y.), vol. 28, no. 3, July 1964, pp. 430-434.

3. Coz, Marcel: Projection Operators for Rearrangement Collisions. Ann. Phys. (N.Y.), vol. 35, no. 1, Oct. 18, 1965, pp. 53-66.

4. Hahn, Yukap: Projection Operators in the Unified Reaction Theory. Phys. Rev., vol. 142, no. 3, Feb. 18, 1966, pp. 603-607.

5. Chen, J. C. Y.; and Mittleman, M. H.: Coupled Equations for Rearrangement Collisions. II. Ann. Phys. (N.Y.), vol. 37, no. 2, Apr. 1, 1966, pp. 264-270.

6. Chen, Joseph C. Y.: Formal Theory of Multichannel Rearrangement Collisions. Phys. Rev., vol. 152, no. 4, Dec. 23, 1966, pp. 1454-1459.

7. Volkin, Howard C.: Generalized Potentials for Inelastic Scattering. Phys. Rev., vol. 155, no. 4, Mar. 20, 1967, pp. 1177-1190. 